\title{
Construção de atributos binários baseada em análise de interações
}

Carlos da Silva dos Santos

\author{
TESE APRESENTADA \\ AO \\ Instituto de MATEMÁtiCA E EstatístiCA \\ DA \\ UniVERSidAdE DE SÃo PAUlo \\ PARA \\ OBTENÇÃO DO TÍTULO \\ $\mathrm{DE}$ \\ DOUTOR EM CIÊNCIAS

\section{Programa: Ciência da Computação \\ Orientador: Prof. Dr. Roberto Hirata Junior}

Durante o desenvolvimento deste trabalho o autor recebeu auxílio financeiro da

FAPESP

São Paulo, julho de 2010 


\section{Construção de atributos binários baseada em análise de interações}

Este exemplar corresponde à redação final da tese devidamente corrigida e defendida por Carlos da Silva dos Santos e aprovada pela Comissão Julgadora.

Banca Examinadora:

- Prof. Dr. Roberto Hirata Junior (orientador) - IME-USP.

- Prof. Dr. Roberto Marcondes Cesar Junior - IME-USP

- Prof. Dr. João Eduardo Kögler Junior - EP-USP

- Prof. Dr. Nelson Delfino D’Avila Mascarenhas - UFSCar

- Prof. Dr. Junior Barrera - FFCLRP 


\section{Agradecimentos}

O presente trabalho não teria sido possível sem a colaboração de inúmeras pessoas, mais do que eu poderia citar aqui. Os meus agradecimentos vão para todos os colegas e amigos do laboratório vision, em especial Arnaldo, David, Jesus e Rodrigo. Ao longo do doutorado, eu tive o prazer de colaborar com Nina Hirata, Luis Roberto de Paula, Carlos Higa e Ronaldo Hashimoto, que me ensinaram muito. Muito obrigado à equipe do Serviço de Informática do Instituto do Coração por ter me acolhido tão bem em parte desse trabalho, em particular para Edson, Giuliana, Luciano, Marcos, Mary Rose e Roberto. Eu aprendi muito com os meus colaboradores do Incor, entre eles: Mauricio, Ramon, Dra. Simone, Dr. Claudio e, especialmente, Marina e Marco Antonio. A todos eles os meus mais profundos agradecimentos. Agradeço ao Flávio Yamamoto pelo companheirismo na preparação para o exame de algoritmos. Sou grato aos professores Fábio Cozman e Roberto Marcondes pelas dicas e observações que levaram a melhora deste trabalho. A meus pais e irmãos, muito obrigado por terem sempre me apoiado nessa jornada, desde o começo. Minha gratidão para Alice, pela compreensão e por seu esforço para que eu chegasse até aqui. Um agradecimento especial para o infinitamente paciente Roberto Hirata Júnior, que se empenhou nessa aventura muito mais do que alguém poderia pedir de um amigo. Por fim, agradeço à Fapesp pelo financiamento deste trabalho. 


\section{Resumo}

Este trabalho trata do problema da construção de atributos para classificação quando atributos e rótulos são binários. A abordagem adotada visa reduzir efeitos de interação entre atributos, amenizando a necessidade dos classificadores lidarem com essas interações. Para tanto, é introduzida uma nova técnica que usa uma matriz de cálculo de paridade para transformar as coordenadas do vetor de atributos binários. Tal matriz permite a manipulação de diversas medidas derivadas da teoria da informação. A transformação resultante induz a formação de grupos de variáveis binárias. Baseando-se nessa técnica, um algoritmo inédito de análise de componentes independentes de variáveis binárias é apresentado, assim como um algoritmo que induz a independência condicional entre os atributos (dado o valor do rótulo). Um terceiro algoritmo apresentado reduz a Informação de Interação entre os atributos, uma medida associada ao grau de redundância ou colaboração entre atributos. Tal algoritmo é empregado no problema do projeto de operadores em dois níveis para imagens, em que múltiplos operadores são combinados para a obtenção de uma imagem final. Nesse caso, o algoritmo apresentado guia a estratégia de divisão de uma imagem em sub-regiões. É apresentado um arcabouço para o projeto de operadores de imagens em dois níveis, incorporando métodos de seleção de atributos e comparação de modelos. Os resultados mostram que o método proposto propicia melhor desempenho, em comparação com operadores de nível único.

Palavras-chave: Reconhecimento de padrões, extração de atributos, processamentos de imagens, teoria da informação, análise de componentes independentes. 


\section{Abstract}

This work addresses the task of feature extraction for classification when both labels and features are binary. Our approach aims to build features with reduced interaction effects, thus relieving the classifiers from dealing explicitly with interactions. We introduce a new technique for building binary features by means of a parity calculating matrix that changes the coordinates of a binary vector. That matrix can be built purposefully for manipulating specific information theoretic measures. The resulting transform gives rise to groups of binary variables. A new algorithm for independent component analysis of binary features is proposed, based on this technique. In the context of classification, a new algorithm is presented that reduces the conditional dependence of features, given the label. A third algorithm presented in this text reduces the Interaction Information between pairs of features, a measure associated with redundancy or collaboration among predictors. This algorithm is used in the context of designing two-level operators for binary images. This type of operator combines the responses of several first-level operators to create the output image. In this case, the interaction reducing algorithm handles the division of the image into subregions. A complete framework for designing these operators is provided, involving feature selection and model comparison techniques. Experimental results show that the proposed method generates more accurate operators, compared to the single-level ones.

Keywords: Pattern recognition, feature extraction, image processing, information theory, independent component analysis. 


\section{Sumário}

Lista de Abreviaturas $\quad$ ix

Lista de Símbolos $\quad$ xi

1 Introdução 1

1.1 Objetivos . . . . . . . . . . . . . . . . . . 4

1.2 Organização do texto $\ldots \ldots \ldots \ldots . \ldots \ldots$

1.3 Contribuições do trabalho . . . . . . . . . . . . . . . . 5

1.4 Lista de publicações . . . . . . . . . . . . . . . . . . . . 5

\begin{tabular}{|lll}
2 & Aprendizado Computacional & $\mathbf{7}$
\end{tabular}

2.1 Conceitos de Teoria da Decisão . . . . . . . . . . . . . . . . . . . 7

2.2 Combinação de Classificadores . . . . . . . . . . . . . . . . 10

\begin{tabular}{|lr}
3 & Tópicos de Teoria da Informação \\
\hline
\end{tabular}

3.1 Entropia . . . . . . . . . . . . . . . . . . . 15

3.2 Informação mútua e entropia relativa $\ldots \ldots \ldots$

3.3 Erro de Bayes e informação mútua . . . . . . . . . . . . . . . 18

3.4 Informação de Interação . . . . . . . . . . . . . . . . . . 18

3.5 Classificador Naive Bayes e interações . . . . . . . . . . . . . . 24

$3.6 \quad$ Análise de Componentes Independentes . . . . . . . . . . . . 27

4 Análise de Componentes Independentes para Variáveis Binárias 29

4.1 Convergência, complexidade e qualidade da solução . . . . . . . . 45

4.2 Experimentos com o algoritmo de ICA binária . . . . . . . . . . 58 
5 Construção de atributos por minimização da multi-informação condicional

5.1 Um algoritmo para a redução da multi-informação condicional . . 69

5.2 Convergência, complexidade e qualidade da solução . . . . . . . . 73

5.3 Experimentos com imagens binárias . . . . . . . . . . . . . 74

6 Construção de Atributos Baseada em Análise de Interações 79

6.1 Transformações de atributos binários e informação de interação. . 79

6.2 Algoritmo para redução de interações . . . . . . . . . . . . 83

6.3 Experimentos . . . . . . . . . . . . . . . . 85

\begin{tabular}{|lll}
\hline & Projeto de filtros de imagens em dois níveis & 89
\end{tabular}

7.1 Operadores de imagens binárias . . . . . . . . . . . . . . . 89

$7.2 \quad$ Projeto de operadores em dois níveis . . . . . . . . . . . . . . . . 90

7.3 Um arcabouço para projeto de operadores em dois níveis . . . . . 92

7.3 .1 Construção de subjanelas . . . . . . . . . . . . 93

7.3 .2 Seleção de Operadores . . . . . . . . . . . . . . . . . . . . 95

7.4 Descrição dos experimentos . . . . . . . . . . . . . . . . 101

7.5 Segmentação de texto em imagens . . . . . . . . . . . . . . . 102

7.6 Reconhecimento de Texturas . . . . . . . . . . . . . . . 103

7.7 Identificação de caracteres . . . . . . . . . . . . . . . 105

7.8 Comparação com janelas selecionadas manualmente . . . . . . . 106

\begin{tabular}{|lr|}
\hline Conclusões & 113
\end{tabular}

8.1 Possíveis extensões . . . . . . . . . . . . . . . . . . 115

\begin{tabular}{lll}
\hline A & Demonstrações & 117
\end{tabular}

A.1 Fórmulas de Teoria da Informação . . . . . . . . . . . . . . . . 117 


\section{Lista de Abreviaturas}

ICA Análise de Componentes Independentes (Independent Component Analysis).

ISI Divisão Incremental de Intervalos (Incremental Splitting of Intervals).

KL Kullback-Leibler.

MAE Erro absoluto médio (Mean Absolute Error).

MDL Comprimento Mínimo de Descrição (Minimum Description Length).

PCA Análise de Componentes Principais (Principal Component Analysis). 


\section{Lista de Símbolos}

$\begin{array}{ll}X & \text { Variável aleatória representando um atributo/preditor. } \\ Y & \text { Variável aleatória representando um rótulo/classe. } \\ x & \text { Valor da variável aleatória } \mathbf{X} . \\ \mathcal{X} & \text { Domínio da variável aleatória } \mathbf{X} . \\ \mathbf{X} & \text { Vetor aleatório de atributos/preditores. } \\ \mathbf{x} & \text { Valor do vetor de atributos } \mathbf{X} \text {. } \\ W & \text { Um conjunto de variáveis binárias ou uma janela, no contexto } \\ & \text { de imagens binárias. } \\ \mathbf{W} & \text { Matriz binária de cálculo de paridade. } \\ \mathbf{w}_{i} & i \text {-ésima linha da matriz W. } \\ \mathbf{I}_{n} & \text { Matriz identidade } n \text { xn } . \\ H(A) & \text { Entropia de Shannon da variável aleatória } A . \\ I(A ; B) & \text { Informação mútua entre variáveis } A \text { e } B . \\ I(A ; B \mid C) & \text { Informação mútua condicional entre variáveis } A \text { e } B, \text { dada variável } C . \\ I(A ; B ; C) & \text { Informação de interação entre variáveis } A, B \text { e } C . \\ M I\left(X_{1}, X_{2}, \ldots, X_{n}\right) & \text { Multi-informação das variáveis } X_{1}, X_{2}, \ldots, X_{n} \\ M I(\mathbf{X}) & \text { Multi-informação dos elementos do vetor aleatório } \mathbf{X} . \\ \theta & \text { Conjunto de parâmetros que especificam um classificador/modelo. } \\ \psi & \text { Uma função Booleana. }\end{array}$




\section{Lista de Figuras}

3.1 Diagramas de informação para atributos redundantes . . . . . . . 20

3.2 Representação do classificador Naive Bayes por uma rede Bayesiana. ......................... 25

3.3 Representação de um classificador TAN por uma rede Bayesiana. 26

$4.1 \quad$ Região permitida para $\tau_{s t}$ quando $\tau_{t}=0.1 \ldots \ldots \ldots$

4.2 Região permitida para $\tau_{s t}$ quando $\tau_{t}=0.25 \ldots \ldots$. . . . 53

4.3 Perda de multi-informação . . . . . . . . . . . . . 56

4.4 Distribuição da perda de multi-informação . . . . . . . . . . . 57

$4.5 \quad$ Entropia de fonte comparada com entropia de saída, $p_{R}=0$. . . . 63

$4.6 \quad$ Entropia de fonte comparada com entropia de saída, $p_{R}=0.01 . \quad 64$

$4.7 \quad$ Entropia de fonte comparada com entropia de saída, $p_{R}=0.02 . \quad 65$

5.1 Imagens de treinamento para reconhecimento de textura . . . . . 75

5.2 Imagem de treinamento para reconhecimento de regiões de texto . 75

5.3 Amostragem de imagens . . . . . . . . . . . . . 76

5.4 Transformação de imagem em vetor . . . . . . . . . . . . 76

5.5 Subjanelas obtidas para minimização de multi-informação condicional . . . . . . . . . . . . . . . . 77

6.1 Imagens de treinamento para identificação de caractere . . . . . . 87

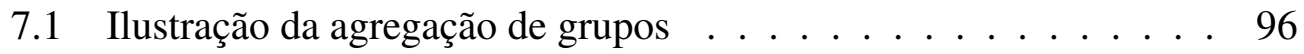

7.2 Janelas obtidas para minimização de interação, segmentação de texto . . . . . . . . . . . . . . . . . . . 103

7.3 MAE para tarefa de segmentação de texto . . . . . . . . . . . 104

7.4 Imagens de segmentação de regiões de texto. . . . . . . . . . . 108 
7.5 Janelas obtidas para minimização de interação, segmentação de textura . . . . . . . . . . . . . . . . . . . 109

7.6 MAE para tarefa de segmentação de textura . . . . . . . . . . . . 109

7.7 Imagens para reconhecimento de textura. . . . . . . . . . . . 110

7.8 Janelas obtidas para minimização de interação, reconhecimento de caractere . . . . . . . . . . . . . . . . . . . 111

7.9 MAE para tarefa de reconhecimento de caractere . . . . . . . 111

7.10 Imagens para identificação de caractere . . . . . . . . . . . . . 112 


\section{Lista de Tabelas}

$4.1 \quad$ Tabela de contingência para $P\left(X_{i}, X_{j}\right)$. . . . . . . . . 32

$4.2 \quad$ Tabela de contingência para $P\left(X_{i}, X_{i} \oplus X_{j}\right)$. . . . . . . . 32

4.3 Permutações e efeito na informação mútua $\ldots . . . . . .38$

$4.4 \quad$ Tabela de contingência para $P\left(X_{s}, X_{t}\right) \ldots \ldots \ldots$

4.5 Exemplo de falha do algoritmo de ICA binária . . . . . . . . . 50

4.6 Tabela de contingência para $P\left(X_{s}, X_{t}\right) \ldots \ldots \ldots$. . . . . 50

4.7 Divisão do espaço de parâmetros de $P\left(X_{s}, X_{t}, X_{u}\right)$ por tipo de distribuição .................... 55

6.1 Variação da informação de interação . . . . . . . . . . . . . . 83

6.2 Interação pré e pós transformação, reconhecimento de texto . . . . 87

6.3 Interação pré e pós transformação, reconhecimento de textura . . . 88

6.4 Interação pré e pós transformação, reconhecimento de caractere . 88

7.1 Comparação entre operadores, reconhecimento de texto. . . . . . 104

7.2 MAE para reconhecimento de textura. . . . . . . . . . . . 105

7.3 Comparação entre operadores, reconhecimento de caractere. . . . 106

7.4 Comparação entre métodos de seleção de janelas . . . . . . . . . 107 


\section{Capítulo 1}

\section{Introdução}

A tarefa de analisar dados, identificar regularidades e criar modelos que possam ser aplicados em novas situações é primordial para a atividade científica. $\mathrm{O}$ campo do Aprendizado Computacional trata da descoberta automática de regularidades por meio de algoritmos computacionais e do emprego dos modelos gerados para atividades como classificação e regressão. Considere, por exemplo, um procedimento para avaliar o risco de um paciente desenvolver uma doença cardíaca. O registro médico do paciente fornece uma série de atributos, tais como: sexo, pressão arterial, peso, altura, ocorrência de tabagismo, coletados de modo a formar um vetor. $\mathrm{O}$ objetivo final dessa tarefa é obter um procedimento computacional que forneça uma avaliação sobre o risco de doença a partir do vetor de atributos do paciente. A abordagem por Aprendizado Computacional para esse problema envolve coletar um número grande de dados retrospectivos de pacientes, acompanhados do diagnóstico (ocorrência ou não de doença cardíaca), constituindo o conjunto de treinamento. Emprega-se então um algoritmo de aprendizado, que se utiliza do conjunto de treinamento para gerar uma função de decisão que mapeia o vetor de atributos em rótulos (prognósticos de ocorrência/não ocorrência de doença).

Formalmente, o classificador deve atribuir um rótulo discreto $Y$,

$$
Y \in \mathcal{Y}=\left\{y_{1}, y_{2}, \ldots, y_{k}\right\}
$$


a uma instância representada por um conjunto de atributos

$$
\mathbf{X}=\left\{X_{1}, X_{2}, \ldots, X_{n}\right\}
$$

Cada atributo $X_{i}$ pertence a um domínio próprio, denominado $\mathcal{X}_{i}$, que pode ser um conjunto discreto ou uma faixa contínua de valores. O domínio do conjunto $\mathrm{X}$ de atributos é denotado por $\mathcal{X}$. No contexto de aprendizado estatístico, tanto $Y$ quanto $\mathrm{X}$ são tratados como variáveis aleatórias, adotando-se como hipótese a existência de uma distribuição de probabilidade conjunta $P(\mathbf{X}, Y)$. A sub-área do aprendizado supervisionado, trata de problemas em que o conjunto de treinamento é formado por pares de exemplos $(\mathbf{X}, Y)$. O algoritmo de aprendizado constrói então uma função de classificação $\Psi: \mathcal{X} \mapsto \mathcal{Y}$ que atribui rótulos a objetos. Inúmeras abordagens já foram propostas para o problema do aprendizado supervisionado de classificadores, entre elas: árvores de decisão, redes neurais, máquinas de vetor de suporte (Support Vector Machines - SVM) e regressão logística.

Uma questão recorrente no aprendizado de classificadores é o problema da dimensionalidade (curse of dimensionality), que se refere a dois fenômenos principais. Em primeiro lugar, o aprendizado de um operador exige mais recursos computacionais conforme o número de atributos utilizados cresce. O segundo fenômeno se refere à perda de precisão estatística à medida que mais atributos são adicionados, considerando-se fixo o número de exemplos de treinamento. Quanto maior o número de atributos utilizados pela função de decisão, maior o tamanho do conjunto de treinamento necessário para se obter uma estimativa com precisão satisfatória. Considere, por exemplo, o problema de estimar $P(\mathbf{X}, Y)$ por meio de um histograma, com um conjunto de treinamento de tamanho fixo $L$. Conforme a dimensão do vetor de atributos aumenta, menor será o número de pontos do conjunto de treinamento contido em cada compartimento do histograma, aumentando a variância da estimativa.

O problema da dimensionalidade é particularmente grave em áreas como bioinformática e medicina, onde geralmente a coleta de exemplos é dispendiosa e os conjuntos de treinamento são tipicamente pequenos. O uso ingênuo de um número grande de atributos conduz ao problema do excesso de ajustamento (over- 
fitting) em que a função de decisão passa a usar características do conjunto de treinamento que são acidentes decorrentes da variabilidade estatística e não expressam uma relação genuína entre atributos e rótulo. $\mathrm{O}$ excesso de ajustamento causa perda de desempenho quando são classificados novos objetos, não pertencentes ao conjunto de treinamento, que não apresentam as mesmas características acidentais deste último.

Uma estratégia para lidar com o problema da dimensionalidade é adotar hipóteses simplificadoras sobre a forma da função de decisão e/ou da distribuição conjunta $P(\mathbf{X}, Y)$ de modo a evitar um procedimentos mais complexos de estimação. Um exemplo dessa estratégia é o classificador denominado Naive Bayes, que adota o seguinte formato para a função de decisão:

$$
\Psi(\mathbf{X})=\arg \max _{y \in \mathcal{Y}} \sum_{i=1}^{n} \log P\left(X_{i} \mid y\right)+\log P(y) .
$$

O classificador Naive Bayes ignora interações entre conjuntos de atributos com dois ou mais elementos. Isso ocasiona um viés, isto é, a função de decisão Naive Bayes pode não convergir para a função ótima, mesmo que o número de exemplos tenda ao infinito. Esse viés é compensado por uma menor variância das estimativas, pois o algoritmo de aprendizado somente tem de lidar com a estimativa unidimensional de $P(Y)$ e estimativas bidimensionais das funções $P\left(X_{i} \mid Y\right)$. O classificador Naive Bayes ilustra que ignorar as interações entre atributos envolve um balanço entre a diminuição da variância e o viés introduzido. A ausência de interações também pode simplificar a tarefa computacional do aprendizado e aumentar a capacidade de compreendermos o modelo obtido, pois tipicamente resulta em um número menor de parâmetros e/ou de atributos envolvidos em cada termo do modelo.

O presente trabalho trata do aprendizado de atributos visando reduzir as interações os mesmos. Dadas duas variáveis aleatórias $X$ e $Y$, dizemos que existe interação entre elas quando o conhecimento do valor de uma delas influi no conhecimento sobre a segunda. $O$ foco principal deste texto é a construção de classificadores, contexto em que um conjunto de atributos preditores $\left\{X_{1}, X_{2}, \ldots, X_{n}\right\}$ é utilizado para fornecer um prognóstico sobre o valor de um rótulo $Y$. Nosso in- 
teresse é analisar como os atributos $X_{i}$ atuam na predição de $Y$ e como eles interagem entre si na tarefa de predição. Um ponto investigado ao longo do trabalho é a possibilidade de agregar os preditores em grupos que interagem fortemente entre si dentro de um mesmo grupo mas que apresentem pouca interação com membros de outros grupos.

Os métodos propostos e os experimentos realizados neste texto se restringem a atributos binários, i.e. pertencentes ao conjunto $\{0,1\}$. Problemas com atributos binários são frequentes em áreas como bioinformática [SSZ ${ }^{+} 98$, Fle04], processamento de documentos, análise de cesta de compras [BMUT97], descoberta de regras de associação [AS94] e paleontologia [KB06].

\subsection{Objetivos}

Um dos objetivos deste trabalho é estudar o papel da interação entre atributos para o aprendizado de classificadores e seu desempenho, usando ferramentas da Teoria da Informação. A partir desse estudo, desenvolver métodos de construção de atributos binários que explorem a existência de interações. Um intuito desses métodos é revelar relações entre os atributos, atuando, por exemplo, em tarefas de análise exploratória da dados. Outro resultado pretendido é que esse métodos se apliquem ao aprendizado de modelos estatísticos simplificados, inspirados pelo classificador Naive Bayes, onde a necessidade de modelar interações entre atributos seja reduzida e o problema de aprendizado possa ser decomposto. Finalmente, pretende-se aplicar tais métodos no aprendizado de operadores entre imagens binárias.

\subsection{Organização do texto}

O restante desse texto é organizado da seguinte forma. O Capítulo 2 introduz conceitos básicos sobre classificadores e apresenta o tópico de combinação de classificadores, que será empregado no projeto de operadores de imagem no Capítulo 7. O Capítulo 3 relembra conceitos básicos de teoria da informação e introduz as medidas de interação empregadas pelos métodos introduzidos nos Capítulos 4, 5, 6, 7. Esse capítulo se encerra com uma discussão sobre o papel das interações no desempenho de classificadores e com a apresentação da técnica de Análise de Componentes Independentes, que será retomada depois no Capítulo 4. 
onde um algoritmo de ICA binária é apresentado e discutido.

O Capítulo 5 apresenta um método para a minimização da dependência condicional entre atributos. No Capítulo 6 é apresentado um novo método para transformação de atributos que permite manipular quantidades relacionadas à interação entre os atributos. No Capítulo 7 essa nova técnica é utilizada na construção de filtros de imagens em dois níveis, que trabalham com uma divisão da imagem em sub-regiões. Esse capítulo apresenta um arcabouço para o projeto de operadores em dois níveis que automatiza algumas tarefas antes realizadas manualmente. $\mathrm{O}$ Capítulo 8 traz conclusões e discute extensões deste trabalho.

\subsection{Contribuições do trabalho}

O presente trabalho introduz um novo método para a construção de atributos binários, por meio de transformações correspondentes a matrizes de cálculo de paridade. Três especializações desse método são apresentadas: a primeira resulta numa espécie de análise de componentes independentes para dados binários. A segunda tenta aproximar os atributos gerados de um modelo probabilístico Naive Bayes. A terceira opera pela manipulação da informação de interação dos atributos. Propriedades como convergência, complexidade e desempenho dos algoritmos propostos são analisadas.

O algoritmo de manipulação da informação de interação é empregado para o aprendizado de operadores de imagens binárias em dois níveis. Um arcabouço completo para o projeto de operadores em dois níveis é apresentado, incluindo um novo método de comparação de modelos usado para selecionar a complexidade dos operadores treinados. São apresentados experimentos que mostram o bom desempenho do arcabouço proposto, que automatiza o projeto dos operadores de dois níveis, eliminando a necessidade de intervenção humana no processo.

\subsection{Lista de publicações}

O presente texto incorpora parte do conteúdo das seguintes publicações:

- Santos, Carlos Silva, Nina Sumiko Tomita Hirata e Roberto Hirata Junior: Two-stage binary image operator design: an approach based on interaction information. In Proceedings of the Brazilian Symposium on Computer 
Graphics and Image Processing, 20 (SIBGRAPI), IEEE Computer Society, 2007.

- Santos, Carlos Silva, e Roberto Hirata Junior: Exploring window selection strategies for two-level binary image operator design. In Proceedings of the ISMM 2007, volume 2, páginas 41-42, Instituto Nacional de Pesquisas Espaciais (INPE), 2007.

- Santos, Carlos Silva, Nina Sumiko Tomita Hirata e Roberto Hirata Junior: An Information Theory framework for two-stage binary image operator design. Pattern Recognition Letters, volume 31, número 4: 297-306, 2010. 


\section{Capítulo 2}

\section{Aprendizado Computacional}

Neste capítulo são introduzidos alguns tópicos de Aprendizado Computacional que são explorados ao longo do texto. A Seção 2.1 trata de alguns conceitos de Teoria da Decisão importantes para a caracterização do desempenho de classificadores. A Seção 2.2 introduz o tema da combinação de classificadores, relevante para a discussão sobre operadores de imagens em dois níveis, apresentada no Capítulo 7

\subsection{Conceitos de Teoria da Decisão}

Nesta seção serão apresentados alguns conceitos básicos da Teoria Estatística de Decisão que são relevantes para caracterizar o desempenho de classificadores, orientando a construção de algoritmos de aprendizado. Uma medida natural para avaliar o desempenho de um classificador é a taxa de erro, definida como a probabilidade do classificador atribuir um rótulo $y_{i}$ a uma instância $\mathrm{x} \in \mathcal{X}$ quando o rótulo verdadeiro de $\mathbf{x}$ é $y_{j},(i \neq j)$ [HTF01, Bis06]. A partir deste ponto, vamos supor que os atributos $X_{i}$ que definem o vetor $\mathrm{X}$ são discretos, a menos que se mencione explicitamente o contrário. Nessas condições a taxa de erro é dada por:

$$
P(\text { erro })=\sum_{\mathbf{x} \in \mathcal{X}} P(\mathbf{x}) \sum_{i=1}^{k} g\left(\Psi(\mathbf{x}), y_{i}\right) P\left(y_{i} \mid \mathbf{x}\right)
$$


Em que $g()$ é uma função que indica os erros de classificação:

$$
g\left(\Psi(\mathbf{x}), y_{i}\right)= \begin{cases}1 & \text { se } \Psi(\mathbf{x}) \neq y_{i} \text { (erro) } \\ 0 & \text { se } \Psi(\mathbf{x})=y_{i} \text { (acerto) }\end{cases}
$$

Para minimizar globalmente a probabilidade de erro se faz necessário minimizar o erro em cada ponto do domínio de $\mathrm{X}$, ou seja:

$$
\forall \mathbf{x} \in \mathcal{X}, \Psi(\mathbf{x})=\arg \min _{y \in \mathcal{Y}}[1-P(y \mid \mathbf{x})]
$$

Segue que, para minimizar a taxa de erro, um classificador deve implementar uma função de decisão $\Psi(\mathbf{X})$ tal que:

$$
\Psi(\mathbf{X})=\arg \max _{y \in \mathcal{Y}} P(y \mid \mathbf{X})
$$

Um classificador que implemente uma função de decisão obedecendo à regra acima é denominado classificador de Bayes [HTF01]. Substituindo a regra de decisão (2.3) na expressão do erro (2.1), concluímos que a menor taxa de erro possível para um classificador é determinada pela seguinte equação, que exprime o denominado erro de decisão de Bayes [DGL96, HTF01]:

$$
P_{\text {Bayes }}(\text { erro })=\sum_{\mathbf{x} \in \mathcal{X}} P(\mathbf{x})\left(1-\max _{y \in \mathcal{Y}} P(y \mid \mathbf{x})\right)
$$

É possível derivar um formato mais conveniente da função de decisão 2.3 aplicando-se o teorema de Bayes:

$$
P(Y \mid \mathbf{X})=\frac{P(\mathbf{X} \mid Y) P(Y)}{P(\mathbf{X})}
$$

Como $P(\mathbf{X})$ independe do valor de $Y$, esse fator não influência a escolha do máximo em (2.3), logo podemos escrever a seguinte regra equivalente:

$$
\Psi(\mathbf{X})=\arg \max _{y \in \mathcal{Y}} P(\mathbf{X} \mid y) P(y)
$$

O termo $P(\mathbf{X} \mid Y)$ é denominado função de verossimilhança (likelihood function), 
sendo denotado no presente texto pelo símbolo $\mathcal{L}$ :

$$
\mathcal{L}(\mathbf{X}, Y)=P(\mathbf{X} \mid Y)
$$

O resultado acima sugere a seguinte abordagem para a construção de classificadores: a partir do conjunto de treinamento, realizar uma estimativa $\hat{P}(Y \mid \mathbf{X})$ da distribuição condicional verdadeira $P(Y \mid \mathbf{X})$ e adotar a regra de classificação da equação (2.3), substituindo $P(Y \mid \mathbf{X})$ por $\hat{P}(Y \mid \mathbf{X})$

$$
\Psi(\mathbf{X})=\arg \max _{y \in \mathcal{Y}} \hat{P}(y \mid \mathbf{X})
$$

O desempenho de classificadores construídos pela abordagem acima depende da qualidade da estimativa $\hat{P}(y \mid \mathbf{X})$, causando dificuldades quando o número de atributos aumenta. Em primeiro lugar, para um número moderado de atributos é provável que muitas das configurações possíveis de pares $\mathbf{x}, y$ não ocorram no conjunto de treinamento. Sendo

$$
\mathbf{X}=\left\{X_{1}, X_{2} \ldots, X_{n}\right\}
$$

um vetor de variáveis aleatórias, o número de configurações possíveis para o par de variáveis $(\mathbf{X}, Y)$ é

$$
|\mathcal{Y}| \prod_{i=1}^{n}\left|\mathcal{X}_{i}\right|
$$

em que $|A|$ denota a cardinalidade de um conjunto $A$. Como o número de configurações possíveis cresce exponencialmente com $n$, seria necessário que o conjunto de treinamento acompanhasse esse ritmo de crescimento para que todas as configurações fossem incluídas no conjunto de treinamento, o que não é prático. Esse fato aponta para uma qualidade que se deseja para os classificadores treinados, a capacidade de generalização, definida como a capacidade do classificador apontar com correção o rótulo para instâncias $\mathrm{x}$ ausentes do conjunto de treinamento. Outro aspecto da dificuldade apontada acima decorre do problema da dimensionalidade (curse of dimensionality). Para um conjunto de treinamento de tamanho fixo, o aumento do número de atributos causa perda de precisão da estimativa $\hat{P}(Y \mid \mathbf{X})$ pois o conjunto de treinamento passa a conter um número pequeno de 
ocorrências de cada configuração $(\mathbf{x}, y)$. Isso aumenta a variabilidade da estimativa $\hat{P}(Y \mid \mathbf{X})$, isto é, se repetirmos o aprendizado do classificador para conjuntos de treinamento diferentes teremos estimativa discrepantes para $\hat{P}(Y \mid \mathbf{X})$.

$\mathrm{Na}$ prática, os algoritmos de aprendizado que trabalham com estimativas de $P(Y \mid \mathbf{X})$ adotam alguma hipótese simplificadora que diminua a complexidade da estimativa, reduzindo assim o número efetivo de parâmetros que precisam ser estimados a partir dos dados. Na Seção 3.5, será examinado o classificador Naive Bayes, que adota a hipótese de independência condicional entre atributos, permitindo assim uma decomposição de $P(Y \mid \mathbf{X})$ em termos dependentes de apenas atributos individuais.

\subsection{Combinação de Classificadores}

Os métodos de combinação de classificadores [KHDM98, Die00] envolvem a construção de um conjunto de classificadores individuais. Novas instâncias $\mathbf{x}$ são classificados pela combinação das predições dos classificadores individuais em uma predição unificada. O método mais usual de combinação das respostas dos classificadores é a votação (que pode ser ponderada). A motivação para o uso desses métodos é que o desempenho da combinação é frequentemente superior ao dos classificadores individuais [KHDM98, Die00]. Para que isso ocorra é necessário que os classificadores tenham um certo nível de precisão (seu desempenho deve ser superior ao que se obteria com escolhas aleatórias dos rótulos) e também que haja diversidade entre os erros dos diferentes classificadores [KHDM98, Die00, BWHY05]. Dietterich [Die00] aponta três razões pelas quais combinações podem funcionar melhor que um classificador individual:

- Estatística: Devido a escassez de exemplos, pode ocorrer que vários classificadores treinados tenham desempenho similar sobre o conjunto de treinamento. A ponderação entre esses classificadores diminui a probabilidade de escolhermos um único classificador com capacidade de generalização sofrível.

- Computacional: Muitos algoritmos de treinamento realizam buscas locais no espaço dos classificadores, sendo possível que o classificador resultante reflita um ótimo local. Isso pode ocorrer mesmo quando há número sufi- 
ciente de exemplos e o problema de precisão de estimativa é amenizado. Nesse caso, a ponderação entre os classificadores pode deslocar a função de decisão de um ótimo local, aumentando o desempenho de classificação.

- De representação: Alguns algoritmos não são capazes de gerar todo o espaço de soluções possíveis durante o treinamento. Mesmo que, em tese, o algoritmo seja capaz de gerar qualquer solução, a escassez de exemplos e a adoção de heurísticas de treinamento impedem que a busca se realize sobre todo o espaço de soluções. Logo, a solução ótima pode se encontrar em alguma região não visitada pelo algoritmo durante o treinamento. A combinação de soluções pode, então, ampliar o espectro de funções representáveis, aproximando melhor a solução ótima.

Os métodos de combinação de classificadores podem ser categorizados em termos de duas características: o mecanismo de combinação e a estratégia de geração de diversidade. Um dos meios de combinação mais usual é a votação. Dado um conjunto de classificadores caracterizado pelas funções de decisão

$$
\Psi_{1}(\mathbf{X}), \Psi_{2}(\mathbf{X}), \ldots, \Psi_{m}(\mathbf{X})
$$

a função de classificação da combinação por votação $\Psi(\mathbf{X})$ é determinada por [KHDM98]:

$$
\Psi(\mathbf{X})=\arg \max _{y} \sum_{i=1}^{m} \alpha_{i} h\left(\Psi_{i}(\mathbf{X}), y\right)
$$

em que os $\alpha_{i}$ são pesos positivos e $h$ é uma função indicadora

$$
h(\Psi(\mathbf{X}), y)= \begin{cases}0 & \text { se } \Psi(\mathbf{X}) \neq y \\ 1 & \text { se } \Psi(\mathbf{X})=y\end{cases}
$$

Um segundo método de combinação é a técnica de generalização por empilhamento (Stacked Generalization) [Wo192]. A técnica recebe esse nome porque trabalha com classificadores "empilhados". Dado um conjunto de classificadores 
caracterizado pelas funções de decisão

$$
\Psi_{1}(\mathbf{X}), \Psi_{2}(\mathbf{X}), \ldots, \Psi_{m}(\mathbf{X})
$$

e um conjunto de pares de treinamento $(\mathbf{x}, y)$, a generalização por empilhamento opera pelo aprendizado de um novo classificador que recebe como entrada as respostas dos operadores $\Psi_{i}$. Em outras palavras, as respostas dos operadores $\Psi_{i}$ são concatenadas, dando origem a um vetor $\mathbf{X}^{\prime}$ :

$$
\mathbf{X}^{\prime}=\left(\Psi_{1}(\mathbf{X}), \Psi_{2}(\mathbf{X}), \ldots, \Psi_{m}(\mathbf{X})\right)
$$

Note-se que cada função de decisão $\Psi_{i}(\mathbf{X})$ mapeia o espaço original de atributos $\mathcal{X}$ no espaço do rótulo $\mathcal{Y}$. Portanto, o domínio do vetor $\mathrm{X}^{\prime}$ é

$$
\mathcal{X}^{\prime}=\mathcal{Y}^{m}
$$

Podemos interpretar $\mathcal{X}^{\prime}$ como um novo vetor de atributos, em um espaço diferente do original. Submetendo cada par $(\mathbf{x}, y)$ do conjunto de treinamento aos classificadores $\Psi_{i}$, é possível gerar um novo conjunto de pares $\left(\mathbf{x}^{\prime}, y\right)$. Na generalização por empilhamento, um novo classificador $\Psi$ é treinado sobre os pares de treinamento resultantes $\left(\mathbf{x}^{\prime}, y\right)$. A generalização por empilhamento permite muita flexibilidade, na medida que qualquer método de geração de diversidade pode ser empregado para gerar os classificadores $\Psi_{i}$. A generalização por empilhamento é a técnica usada neste texto para gerar operadores de imagens em dois níveis, no Capítulo7.

Quanto à geração de diversidade, um esquema possível é a manipulação direta dos exemplos. Nesse cenário o algoritmo de aprendizado é executado diversas vezes, a cada vez com um conjunto diferente de exemplos. Essa técnica funciona melhor com algoritmos de aprendizado instáveis, em que uma pequena modificação do conjunto de treinamento pode resultar em mudança significativa da função de decisão [Die00].

Outra maneira de induzir variedade nos classificadores individuais é pela manipulação dos atributos de entrada [Die00]. Por exemplo, os atributos podem ser divididos em subconjuntos, cada um deles sendo utilizado para treinar um clas- 
sificador diferente. Duin e Tax [DT00] realizaram uma série de experimentos de combinação de classificadores, usando diferentes regras de combinação, tipos de classificadores e estratégias de treinamento. Nos experimentos realizados, a estratégia de treinar o mesmo tipo de classificador usando diferentes conjuntos de atributos para posterior combinação se mostrou mais efetiva que a de combinar diferentes tipos de classificadores treinados sobre o mesmo conjunto de atributos [DT00]. Os operadores de imagens binárias apresentados neste texto (capítuld7) usam a estratégia de variar os conjuntos de atributos para criar diversidade. 


\section{Capítulo 3}

\section{Tópicos de Teoria da Informação}

Neste capítulo serão apresentados de maneira resumida alguns conceitos de teoria de informação utilizados ao longo do restante do texto, incluindo as funções de multi-informação e a informação de interação. A primeira mede a redundância de um conjunto de atributos e tem papel essencial em métodos de Análise de Componentes Independentes [Com94], como aquele que será introduzido no Capítulo 4. A segunda indica padrões de interação entre atributos para a predição de um rótulo. O método proposto no Capítulo 6 manipula essa função para obter atributos com baixa interação.

O capítulo conclui com dois tópicos em que a teoria da informação desempenha um pequeno papel. A Seção 3.5 discute o classificador Naive Bayes e algumas de duas variações em termos do papel das interações na construção e no desempenho dos classificadores. A Seção 3.6 introduz o tópico da Análise de Componentes Independentes, que depois será retomado no Capítulo 4, onde será introduzido um método de Análise de Componentes Independentes para dados binários.

\subsection{Entropia}

Para uma variável aleatória discreta $X \in \mathcal{X}$, com distribuição $P(X)$, a entropia [Sha48] é uma função que mede a incerteza média sobre o valor de $X$, definida por [Sha48, CT91]:

$$
H(X)=-\sum_{x \in \mathcal{X}} P(x) \log P(x)
$$


A definição se estende para múltiplas variáveis, resultando na entropia conjunta [Sha48, CT91]:

$$
H\left(X_{1}, X_{2}, \ldots X_{n}\right)=-\sum_{\mathcal{X}_{1}, \ldots, \mathcal{X}_{n}} P\left(x_{1}, x_{2}, \ldots, x_{n}\right) \log P\left(x_{1}, x_{2}, \ldots, x_{n}\right)
$$

A entropia condicional mede a incerteza restante sobre o valor de uma variável $Y \in \mathcal{Y}$ após a observação do valor de uma segunda variável $X$, sendo definida por [CT91, Yeu02]

$$
H(Y \mid X)=-\sum_{x \in \mathcal{X}} \sum_{y \in \mathcal{Y}} P(x, y) \log P(y \mid x)
$$

Pode-se demonstrar que(ver apêndice A.1] [CT91, Yeu02]:

$$
H(Y \mid X)=H(X, Y)-H(X)
$$

\subsection{Informação mútua e entropia relativa}

A informação mútua $I(X ; Y)$ é dada pela diferença entre a incerteza total sobre $Y$ e a incerteza residual após a observação de $X$ [CT91]:

$$
I(X ; Y)=H(Y)-H(Y \mid X)
$$

Aplicando-se a fórmula (3.3), é possível concluir que [CT91]:

$$
I(X ; Y)=H(Y)+H(X)-H(X, Y)
$$

e, notando que

$$
H(X \mid Y)=H(X, Y)-H(Y)
$$

temos

$$
I(X ; Y)=H(X)-H(X \mid Y) .
$$

Portanto, a informação mútua é simétrica [CT91]:

$$
I(X ; Y)=I(Y ; X)
$$


Outra maneira de interpretar a informação mútua é como uma espécie de distância entre a distribuição conjunta $P(X, Y)$ e a distribuição que as variáveis teriam caso fossem independentes: $P(X) P(Y)$. Para isso, introduzimos o conceito de divergência de Kullback-Leibler ou entropia relativa entre duas distribuições $P(X)$ e $Q(X)$ [CT91]:

$$
D(P \| Q)=\sum_{x \in \mathcal{X}} P(x) \log \frac{P(x)}{Q(x)}
$$

$\mathrm{Na}$ definição acima, foram adotadas as seguintes convenções, baseadas em argumentos de continuidade [CT91]:

$$
\begin{gathered}
0 \log (0 / q)=0 \\
p \log (p / 0)=\infty,
\end{gathered}
$$

para $p, q \neq 0$

A divergência de Kullback-Leibler (KL) pode ser interpretada como uma distância entre as distribuições $P$ e $Q$, embora não seja uma métrica (pois é assimétrica e não obedece à desigualdade triangular) [CT91]. A divergência KL é não negativa e se iguala a zero se e somente se $P=Q$ [CT91, Mac03].

Pode-se demonstrar que (ver apêndice A.1)

$$
D(P(X, Y) \| P(X) P(Y))=I(X ; Y) .
$$

Quando $X$ e $Y$ são independentes, temos $P(X, Y)=P(X) P(Y)$ e a informação mútua se anula.

Uma possível extensão multidimensional da informação mútua é dada pela divergência KL entre a distribuição conjunta e a distribuição formada pelo produto das marginais:

$$
\begin{aligned}
M I\left(X_{1}, X_{2}, \ldots, X_{n}\right) & =\sum_{\mathcal{X}_{1}, \ldots, \mathcal{X}_{n}} P\left(x_{1}, x_{2}, \ldots, x_{n}\right) \log \frac{P\left(x_{1}, x_{2}, \ldots, x_{n}\right)}{\prod_{r=1}^{n} P\left(x_{r}\right)} \\
& =\sum_{i=1}^{n} H\left(X_{i}\right)-H\left(X_{1}, X_{2}, \ldots, X_{n}\right)
\end{aligned}
$$


A função definida pela expressão (3.8) é denominada multi-informação [SV98] ou redundância. Na literatura sobre Análise de Componentes Independentes [Com94], a mesma função é também chamada de informação mútua multivariada [HKO01]. Em decorrência das propriedades da divergência KL, a multi-informação é sempre não negativa e se torna nula se e somente se as variáveis envolvidas $\left(X_{1}, X_{2}, \ldots X_{n}\right)$ forem estatisticamente independentes.

\subsection{Erro de Bayes e informação mútua}

Os dois resultados seguintes são interessantes para a construção de atributos envolvendo quantidades da teoria da informação.

O erro de Bayes pode ser limitado em termos da informação mútua entre $Y$ e X por meio da seguinte relação [HR70, Lin91, ST99]:

$$
P_{\text {Bayes }}(\text { erro }) \leq \frac{1}{2}(H(Y)-I(\mathbf{X} ; Y))
$$

Para que a expressão acima seja válida, é necessário que entropia e informação mútua sejam medidas em bits, i.e., usando logaritmo base 2. Esse resultado mostra que a maximização da informação mútua entre atributos e rótulo diminui o valor máximo que o erro de Bayes pode atingir. O próximo resultado mostra que esse limitante não pode ser melhorado por meio de transformações sobre um conjunto fixo de atributos. Seja $Z=\xi(X)$ uma variável aleatória obtida por uma transformação $\xi$ sobre $X$. A desigualdade de processamento de dados (Data Processing Inequality) [CT91] afirma que $Z$ não pode conter mais informação sobre $Y$ do que a variável original $X$ :

$$
I(Z ; Y) \leq I(X ; Y)
$$

\subsection{Informação de Interação}

A Informação de Interação [McG54, Bel03, JB03] é outra extensão possível do conceito de informação mútua para mais de duas variáveis. Quando calculada para um conjunto de três variáveis aleatórias, a informação de interação mede o grau de interação entre duas variáveis aleatórias para a determinação do valor da terceira variável. Sejam $Y, X_{1}$ e $X_{2}$ variáveis aleatórias discretas definidas 
em conjuntos $\mathcal{Y}, \mathcal{X}_{1}$ e $\mathcal{X}_{2}$, respectivamente. A informação de interação de três variáveis $I\left(Y ; X_{1} ; X_{2}\right)$ é definida como [McG54, Bel03, JB03]:

$$
\begin{aligned}
I\left(Y ; X_{1} ; X_{2}\right)= & -H\left(X_{1}\right)-H\left(X_{2}\right)-H(Y)+H\left(X_{1}, X_{2}\right)+H\left(X_{1}, Y\right) \\
& +H\left(X_{2}, Y\right)-H\left(X_{1}, X_{2}, Y\right)
\end{aligned}
$$

É possível manipular a Equação 3.11 de modo a reescrever a informação de interação usando termos de informação mútua[JB03] (ver apêndice A.3):

$$
I\left(Y ; X_{1} ; X_{2}\right)=I\left(Y ;\left(X_{1}, X_{2}\right)\right)-I\left(Y ; X_{1}\right)-I\left(Y ; X_{2}\right)
$$

onde $I\left(Y ;\left(X_{1}, X_{2}\right)\right)$ é a informação mútua entre $Y$ e o par $\left(X_{1}, X_{2}\right)$ considerado conjuntamente.

A informação de interação pode ser negativa ou positiva. Ela se torna negativa quando:

$$
I\left(Y ;\left(X_{1}, X_{2}\right)\right)<I\left(Y ; X_{1}\right)+I\left(Y ; X_{2}\right) .
$$

O fato da informação total que os atributos fornecem $I\left(Y ;\left(X_{1}, X_{2}\right)\right)$ ser menor que a soma das informações individuais indica que os atributos são (ao menos parcialmente) redundantes quanto à determinação de $Y$.

A informação de interação é positiva quando temos:

$$
I\left(Y ;\left(X_{1}, X_{2}\right)\right)>I\left(Y ; X_{1}\right)+I\left(Y ; X_{2}\right) .
$$

Nesse caso, a observação conjunta de $X_{1}$ e $X_{2}$ fornece informação nova sobre $Y$, isto é, informação que não estava contida nos termos $I\left(Y ; X_{1}\right)$ e $I\left(Y ; X_{2}\right)$. A relação entre as quantidades envolvidas na definição da Informação de Interação pode ser visualizada por meio de uma sequência de diagramas de informação [Yeu02], como na Figura 3.1. Um diagrama de informação é similar a um diagrama de Venn, adaptado para que as entropias de variáveis sejam representadas como conjuntos. A intersecção entre dois conjuntos denota informação mútua e a união entre conjuntos denota entropia conjunta.

A informação de interação se relaciona à capacidade de criarmos modelos simplificados de classificadores. Quando $I\left(Y ; X_{1} ; X_{2}\right)=0$ podemos escrever 


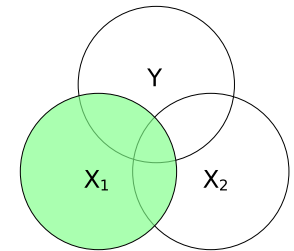

(a)

$H\left(X_{1}\right)$

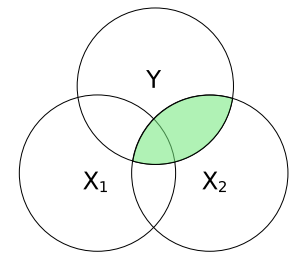

(e)

$I\left(Y ; X_{2}\right)$

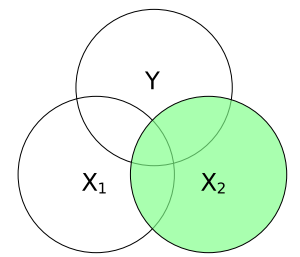

(b)

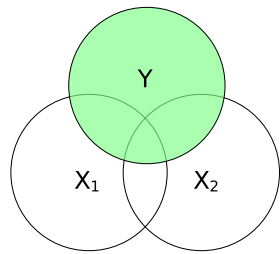

(c)

$H(Y)$

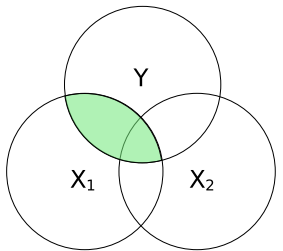

(d)

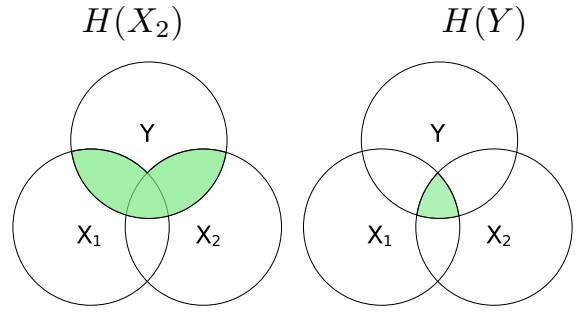

$I\left(Y ; X_{1}\right)$

Figura 3.1: Sequência de diagramas de informação para o caso em que $X_{1}$ e $X_{2}$ são parcialmente redundantes para a determinação de $Y$. Em cada diagrama, a área destacada mostra uma das grandezas definidas no texto.

$P\left(Y \mid X_{1}, X_{2}\right)$ como (ver apêndice A.1)

$$
P\left(Y \mid X_{1}, X_{2}\right)=\frac{1}{P(Y)} P\left(Y \mid X_{1}\right) P\left(Y \mid X_{2}\right) .
$$

Logo, $P\left(Y \mid X_{1}, X_{2}\right)$ pode ser reescrita como o produto de duas funções $f_{1}\left(X_{1}, Y\right)$ e $f_{2}\left(X_{2}, Y\right)$ que medem separadamente a influência de cada atributo sobre $Y$ :

$$
P\left(Y \mid X_{1}, X_{2}\right)=f_{1}\left(X_{1}, Y\right) f_{2}\left(X_{2}, Y\right) .
$$

Assim, a função de decisão do classificador pode ser escrita como uma combinação de termos que dependem apenas dos atributos individuais $X_{1}$ e $X_{2}$, não contendo nenhum termo que meça a interação entre atributos.

Quando a interação é negativa, existe redundância entre $X_{1}$ e $X_{2}$ e uma decomposição como a da Equação (3.14) corre o risco de contar duas vezes a mesma evidência sobre $Y$. Por exemplo, imagine um classificador Naive Bayes com dois atributos $X_{1}$ e $X_{2}$, em que ambos sejam realmente independentes condicionalmente, isto é:

$$
P\left(X_{1}, X_{2} \mid Y\right)=P\left(X_{1} \mid Y\right) P\left(X_{2} \mid Y\right) \text {. }
$$


Então podemos escrever a função de decisão de maneira exata como

$$
\Psi(\mathbf{X})=\arg \max _{y \in \mathcal{Y}}\left\{\log P\left(X_{1} \mid y\right)+\log P\left(X_{2} \mid y\right)+\log P(y)\right\}
$$

e obter um resultado ótimo para esse par de atributos. Suponha que se adicione um terceiro atributo $X_{3}$ que seja uma cópia de $X_{1}$. Obviamente, ambos atributos são completamente redundantes e

$$
I\left(X_{1} ; X_{3} ; Y\right)<0
$$

A função de decisão do classificador Naive Bayes com o atributo adicional seria

$$
\Psi^{\prime}(\mathbf{X})=\arg \max _{y \in \mathcal{Y}}\left\{\log P\left(X_{1} \mid y\right)+\log P\left(X_{2} \mid y\right)+\log P\left(X_{3} \mid y\right)+\log P(y)\right\}
$$

$\operatorname{mas} P\left(X_{1} \mid y\right)=P\left(X_{3} \mid y\right)$, então

$$
\Psi^{\prime}(\mathbf{X})=\arg \max _{y \in \mathcal{Y}}\left\{2 \log P\left(X_{1} \mid y\right)+\log P\left(X_{2} \mid y\right)+\log P(y)\right\}
$$

Vamos chamar de $q(\mathbf{X}, y)$ a função maximizada na expressão 3.15 , isto é:

$$
q(\mathbf{X}, y)=\log P\left(X_{1} \mid y\right)+\log P\left(X_{2} \mid y\right)+\log P(y)
$$

Do mesmo modo, denotaremos por $q^{\prime}(\mathbf{X}, y)$ a função maximizada na expressão 3.16

$$
q^{\prime}(\mathbf{X}, y)=2 \log P\left(X_{1} \mid y\right)+\log P\left(X_{2} \mid y\right)+\log P(y)
$$

Fazendo $q^{\prime}(\mathbf{X}, y)-q(\mathbf{X}, y)$, obtemos

$$
q^{\prime}(\mathbf{X}, y)-q(\mathbf{X}, y)=\log P\left(X_{1} \mid y\right)
$$

O termo $\log P\left(X_{1} \mid y\right)$ aparece em excesso, ou seja, a evidência fornecida por $X_{1}$ tem maior peso na função de decisão $\Psi^{\prime}(\mathbf{X})$ do que na função ótima $\Psi(\mathbf{X})$, devido à introdução da duplicata $X_{3}$. Há casos em que essa contagem adicional da mesma 
evidência não prejudica o classificador e temos

$$
\Psi^{\prime}(\mathbf{X})=\Psi(\mathbf{X})
$$

Domingos e Pazzani [DP97] mostraram dois exemplos em que isso ocorre: o primeiro quando a função de decisão é uma conjunção de atributos binários, o segundo quando a função de decisão é uma disjunção de atributos binários. Porém, a contagem adicional de evidência pode alterar a decisão tomado pelo classificador. Por exemplo, considerando um rótulo binário,

$$
y \in\{0,1\}
$$

vamos supor uma instância do vetor de atributos $\mathbf{x}=\left(x_{1}, x_{2}\right)$, tal que

$$
q(\mathbf{x}, y=0)-q(\mathbf{x}, y=1)=\varepsilon
$$

para uma valor $\varepsilon>0$. Como

$$
q(\mathbf{x}, y=0)>q(\mathbf{x}, y=1)
$$

segue que a decisão correta para esta instância $\mathrm{x}$ do vetor de atributos é (pela regra 3.15

$$
\Psi(\mathbf{x})=0 .
$$

Da Equação 3.17), podemos escrever:

$$
q^{\prime}(\mathbf{x}, y=0)=q(\mathbf{x}, y=0)+\log P\left(X_{1} \mid y=0\right)
$$

e

$$
q^{\prime}(\mathbf{x}, y=1)=q(\mathbf{x}, y=1)+\log P\left(X_{1} \mid y=1\right) .
$$

Calculando-se a diferença entre as duas expressões acima, obtemos $q^{\prime}(\mathbf{x}, y=0)-q^{\prime}(\mathbf{x}, y=1)=q(\mathbf{x}, y=0)-q(\mathbf{x}, y=1)+\log P\left(X_{1} \mid y=0\right)-\log P\left(X_{1} \mid y=1\right)$ 
Aplicando a igualdade (3.18), temos

$$
q^{\prime}(\mathbf{x}, y=0)-q^{\prime}(\mathbf{x}, y=1)=\varepsilon+\log P\left(X_{1} \mid y=0\right)-\log P\left(X_{1} \mid y=1\right) .
$$

Caso

$$
\log P\left(X_{1} \mid y=0\right)-\log P\left(X_{1} \mid y=1\right)<-\varepsilon,
$$

nós teremos

$$
q^{\prime}(\mathbf{x}, y=0)-q^{\prime}(\mathbf{x}, y=1)<0
$$

e de acordo com a regra (3.16) a função de decisão será dada por

$$
\Psi^{\prime}(\mathbf{x})=1
$$

Para essa instância específica $\mathbf{x}$, a função de decisão $\Psi^{\prime}(\mathbf{x})$ contraria a função ótima $\Psi(\mathbf{x})$, causando perda de desempenho do classificador. Isso mostra que a existência de interação negativa pode prejudicar o classificador Naive Bayes. Para evitar essa degradação seria preciso que função de decisão compensasse a redundância dos atributos, o que não é possível com o classificador Naive Bayes clássico.

No caso em que $I\left(Y ; X_{1} ; X_{2}\right)>0$, simplificações que ignorem as interações causam perda de informação sobre $Y$. Por exemplo, suponha dois atributos binários $X_{1}$ e $X_{2}$ gerados independentemente, com

$$
P\left(X_{1}=1\right)=P\left(X_{2}=1\right)=0.5
$$

valendo a seguinte relação entre atributos e rótulo

$$
Y=X_{1} \oplus X_{2}
$$

em que $\oplus$ denota a operação OU-exclusivo. Não é possível afirmar nada sobre o valor de $Y$ conhecendo somente $X_{1}$. Da mesma forma, $X_{2}$ também não tem poder se predição sobre $Y$. Logo,

$$
I\left(Y ; X_{1}\right)=0
$$


e

$$
I\left(Y ; X_{2}\right)=0 .
$$

Mas o rótulo é totalmente determinado se soubermos o valor de ambos os atributos, então

$$
I\left(Y ;\left(X_{1}, X_{2}\right)\right)>0 .
$$

Segue que $I\left(Y ; X_{1} ; X_{2}\right)>0$. Nessa situação, qualquer classificador que leve em conta somente contribuições individuais de atributos não será capaz de predizer o valor de $Y$. Seria necessário incluir termos que considerem a ocorrência conjunta de $X_{1}$ e $X_{2}$.

Em resumo, valores negativos de informação de interação exprimem redundância entre atributos. Valores positivo indicam cooperação entre os atributos para determinar o valor do rótulo. Mas ambos os casos favorecem classificadores que levem em conta explicitamente a ocorrência de interações em detrimento de modelos mais simplificados.

\subsection{Classificador Naive Bayes e interações}

Numa tentativa de lidar com o excesso de ajustamento e o problema da dimensionalidade, o classificador Naive Bayes adota uma hipótese de independência entre atributos, condicionalmente à observação da classe:

$$
P(\mathbf{X} \mid Y)=\prod_{i=1}^{n} P\left(X_{i} \mid Y\right)
$$

A distribuição $P(\mathbf{X}, Y)$ correspondente ao modelo adotado pelo classificador Naive Bayes pode ser representado por uma Rede Bayesiana [Mur01, Jor04, Bis06], um grafo direcionado acíclico em que os vértices representam variáveis aleatórias e as arestas representam relações de dependência estatística entre variáveis. Um grafo representando um classificador Naive Bayes é mostrado na Figura 3.2 .

A função de decisão do classificador Naive Bayes é derivada a partir da expressão 3.22 e pode ser expressa de maneira conveniente por

$$
\Psi(\mathbf{X})=\arg \max _{y \in \mathcal{Y}} \sum_{i=1}^{n} \log P\left(X_{i} \mid y\right)+\log P(y)
$$




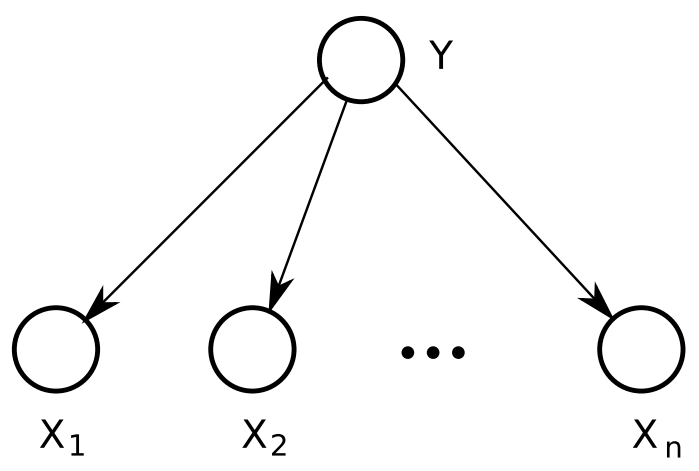

Figura 3.2: Representação do classificador Naive Bayes por uma rede Bayesiana.

O classificador Bayes ingênuo ignora interações entre os atributos, tanto durante o aprendizado do classificador quanto durante sua aplicação. Apesar disso, esse método é bastante efetivo e frequentemente oferece precisão comparável a métodos pertencentes ao estado da arte [Fle04]. Domingos e Pazzani [DP97] analisaram o classificador Naive Bayes, mostrando que este pode aprender a função de classificação ótima mesmo quando a independência não se verifica. São exemplos os casos em que a função ótima é uma conjunção ou disjunção de atributos. No mesmo trabalho [DP97], experimentos de classificação com 28 conjuntos de dados apontaram uma tendência de melhor desempenho do classificador Naive Bayes em relação a árvores de decisão, mesmo quando existe alto grau de dependência entre atributos [DP97].

A efetividade do classificador Naive Bayes motivou a busca por modelos que conservassem uma forma simples fatorada para $P(\mathbf{X} \mid Y)$, ao mesmo tempo permitindo que interações de pequena ordem entre os atributos fossem integradas [FGG97, JB03, ZL03]. O classificador Naive Bayes aumentado em árvore (Tree Augmented Naive Bayes - TAN) [FGG97] adota uma fatoração em que interações entre pares de atributos são incorporadas ao modelo:

$$
\log P(\mathbf{X} \mid Y)=\sum_{i, j \in \mathcal{T}} \log \frac{P\left(X_{i}, X_{j} \mid Y\right)}{P\left(X_{i} \mid Y\right) P\left(X_{j} \mid Y\right)}+\sum_{i=1}^{n} \log P\left(X_{i} \mid Y\right)
$$

A distribuição adotada pelo classificador TAN também pode ser representada por uma rede Bayesiana. A Figura 3.3 mostra um exemplo de classificador TAN com quatro atributos: $\left(X_{1}, X_{2}, X_{3}, X_{4}\right)$. O nome do classificador se justifica porque o 
subgrafo contendo somente os atributos $X_{i}$ forma uma árvore.

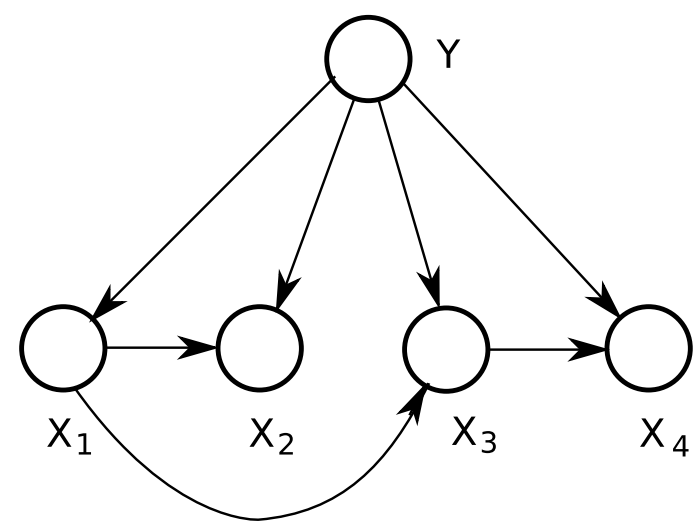

Figura 3.3: Representação de um classificador TAN por uma rede Bayesiana.

A árvore do classificador TAN é construída da seguinte forma. Seja $G$ o grafo completo em que os nós são todos os atributos $X_{i}$. A aresta entre os atributos $X_{i}$ e $X_{j}$ recebe um peso igual à informação mútua condicional entre os atributos:

$$
I\left(X_{i} ; X_{j} \mid Y\right)=H\left(X_{i} \mid Y\right)+H\left(X_{j} \mid Y\right)-H\left(X_{i} ; X_{j} \mid Y\right)
$$

A árvore do classificador é então definida com a árvore geradora de peso máximo de $G$. Pode-se demonstrar que a árvore construída dessa forma corresponde a um estimador de máxima verossimilhança da distribuição $P(\mathbf{X} \mid Y)$, restrito a um espaço de modelos que fatoram como árvores [CL68, FGG97]. Os experimentos de Friedman et al [FGG97] mostram que o classificador TAN apresenta desempenho superior ao Naive Bayes em alguns conjuntos, e raramente apresenta desempenho inferior.

Jakulin e Bratko [JB03] propuseram o uso da informação de interação para a construção de atributos utilizados por um classificador Naive Bayes. O procedimento proposto parte do cálculo da informação de interação para todos os pares de atributos $\left(X_{i}, X_{j}\right), i \neq j$. Em seguida, as interações de maior magnitude (tanto negativas quanto positivas) entre pares de atributos são "resolvidas": um novo atributo $Z_{k}=\left(X_{i}, X_{j}\right)$ é criado para cada par de atributos $\left(X_{i}, X_{j}\right)$ que apresentar interação significativa. Um procedimento de seleção de atributos é aplicado ao novo espaço de atributos $X_{1}, \ldots, X_{n}, Z_{1}, \ldots, Z_{m}$, em que $m$ é o número de 
interações "resolvidas" pelo procedimento. Em seguida, um classificador Naive Bayes é derivado sobre o conjunto de atributos selecionado. O procedimento é justificado pelo princípio de que uma interação significativa entre dois atributos indica que ambos devem ser observados conjuntamente. Jakulin e Bratko apresentam resultados comparando [JB03] o classificador Naive Bayes produzido com os atributos originais e o classificador Naive Bayes produzido a partir do conjunto de atributos derivado com o procedimento descrito, concluindo que o procedimento de construção de atributos geralmente melhora o desempenho de classificação.

Os métodos citados nesta seção indicam que modelos simplificados podem ter desempenho compatível com métodos mais sofisticados, pois amenizam o problema da dimensionalidade. Eles também mostram que interações de pequena ordem, entre pares de atributos, têm impacto relevante no desempenho de classificadores. No classificador TAN, as interações são incorporadas ao classificador pela inclusão de termos dependentes de $P\left(X_{i}, X_{j} \mid Y\right)$, em que $\left(X_{i}, X_{j}\right)$ são pares de atributos. No método de Jakulin e Bratko [JB03], o conjunto de atributos é estendido com a adição de atributos formados pelo produto cartesiano de dois atributos. Indiretamente, isso também leva à estimativa de termos do tipo $P\left(X_{i}, X_{j} \mid Y\right)$ quando o classificador é treinado. O Capítulo 6 apresenta uma nova maneira de construir atributos binários por meio de transformações sobre variáveis aleatórias, levando em consideração as interações entre atributos.

\subsection{Análise de Componentes Independentes}

A análise de componentes independentes [Com94] (Independent Component Analysis - ICA) é uma técnica de extração de atributos geralmente empregada para dados contínuos, embora existam algumas variações que se aplicam a dados discretos [HH01, BJ04]. O Capítulo 4 introduz um novo algoritmo para ICA no contexto de dados binários.

Dado um vetor $\mathbf{X}=X_{1}, X_{2}, \ldots, X_{n}$, com componentes $X_{i} \in \mathbb{R}$, o objetivo da ICA é encontrar uma transformação linear

$$
\mathbf{Z}=\mathbf{W X}
$$

em que $\mathbf{Z} \in \mathbb{R}^{m}, m \leq n$ e $W$ é uma matriz $m \times n$, de modo que a multi-informação 
entre os componentes de $\mathbf{Z}$ seja minimizada. No caso limite, a multi-informação $M I(\mathbf{Z})$ tende a zero e os componentes $Z_{i}$ se tornam independentes entre si, justificando o nome do método.

A ICA foi primeiramente desenvolvida no contexto da separação cega de sinais (ver, por exemplo [BS95]), um problema em que sinais independentes, chamados "fontes" se combinam linearmente, sendo medidos por um conjunto de sensores. 


\section{Capítulo 4}

\section{Análise de Componentes Independentes para Variáveis Binárias}

Neste capítulo será apresentado um método novo para Análise de Componentes Independentes (ICA) [Com94] quando as variáveis analisadas são binárias (i.e. pertencentes ao conjunto $\{0,1\}$ ). Junto com o algoritmo de ICA binária, o presente capítulo apresenta uma técnica de transformação de dados binários utilizando matrizes de paridade. Essa técnica servirá de base para métodos de extração de atributos para dados binários apresentadas em capítulos posteriores. Dados binários são relevantes em áreas tão diversas quanto bioinformática [SSZ ${ }^{+}$98, Fle04], processamento de documentos, análise de cesta de compras [BMUT97], descoberta de regras de associação [AS94] e paleontologia [KB06]. É importante dispor de ferramentas que considerem particularidades desse tipo de dados em atividades como análise exploratória de dados, construção de atributos e restauração (denoising) de dados. A Análise de Componentes Independentes é uma candidata interessante para a análise de dados binários, devido ao sucesso obtido com outros tipos de dados [LC03, JrE03, SKAG04]. Pajunen [Paj97] propôs um método de ICA para extrair fontes binárias a partir de dados contínuos (i.e. números reais) mas a primeira proposta de aplicação de ICA para dados binários se deve a Himberg e Hyvärinen [HH01]. Assim como em outras técnicas [SH06, $\mathrm{KPS}^{+}$08] de análise de dados binários, o método proposto em [HH01] assume um modelo em que as variáveis latentes (as "fontes", no jargão de ICA) binárias são combinadas por meio da função Booleana OR, de acordo com um vetor de coeficientes binários. Esse modelo é descrito pela 
seguinte equação Booleana:

$$
x_{i}=\bigvee_{j=1}^{m} a_{i j} \wedge y_{j}, i=1,2 \ldots, n,
$$

onde $x_{i}$ é um elemento do vetor de dados observados, $y_{j}$ representa a $j$-ésima variável latente e o coeficiente binário $a_{i j}$ indica se tal variável latente contribui para o elemento $x_{i}$. Visando evitar um problema de otimização combinatória, Himberg e Hyvärinen [HH01] aplicam um algoritmo usual de ICA (i.e. para variáveis contínuas) sobre os dados binários, seguindo-se a aplicação de um limiar (thresholding) aos coeficientes e às fontes para obter-se parâmetros binários. A justificativa é que esse procedimento serve como aproximação do modelo da Equação (4.1) quando os dados são esparsos (ou seja, cada fonte tem uma pequena probabilidade a priori de estar ativa). Buntine e Jakulin [BJ04] introduziram um algoritmo de ICA para dados discretos, aplicável também para dados binários. O algoritmo [BJ04] baseia-se num modelo probabilístico em que os dados observados são misturas aditivas de variáveis multinomiais (variáveis de Bernoulli, no caso binário). Posteriormente, Kabán e Bingham [KB06] propuseram um modelo probabilístico em que as fontes independentes binárias são modeladas como variáveis aleatórias de distribuição beta. Como o modelo é intratável numericamente, os autores adotam [KB06] uma aproximação variacional para a inferência de parâmetros do modelo. Todos os algoritmos citados baseiam-se em combinações aditivas (do tipo OR) entre as fontes. Ou seja, se a ativação de uma fonte contribui para ativar um determinado elemento do vetor observado, a ativação simultânea de uma segunda fonte pode somente reforçar esse efeito e nunca contribuir para diminuir a probabilidade de ativação desse elemento.

O novo método de ICA para dados binários introduzido no presente capítulo diferencia-se dos métodos citados anteriormente por se basear num modelo em que as variáveis latentes (fontes) são obtidas pelo cálculo de funções de paridade sobre os dados observados. Mais apropriadamente, o método proposto aqui poderia ser classificado como um método de componentes minimamente dependentes (least dependent components), denominação proposta por Stögbauer $e t$ al. [SKAG04] para métodos em que não se assume explicitamente a independên- 
cia estatística entre as variáveis latentes mas apenas se busca minimizar a dependência estatística entre as mesmas. No entanto, o termo guarda-chuva "Análise de Componentes Independentes" foi o que prevaleceu para denominar toda essa família de métodos e, portanto, será utilizado neste texto.

O método a ser apresentado abaixo foi inspirado por dois algoritmos iterativos de ICA [LMI03, SKAG04] que se dedicam a um subconjunto de variáveis a cada iteração, buscando minimizar a multi-informação das variáveis a cada passo iterativo, em contraste com os métodos usuais que otimizam a multi-informação globalmente sobre todas as variáveis a cada iteração [BS95, Hyv99, HO00]. Esses algoritmos iterativos [LMI03, SKAG04] se aproveitam de uma decomposição conveniente da multi-informação em função da transformação linear a que as variáveis são submetidas. Quando essa transformação linear se limita a rotações em $\mathbb{R}^{n}$, a multi-informação pode ser decomposta em duas parcelas, a primeira dependente apenas de um par de variáveis, sendo a segunda parcela invariante à rotação aplicada a esse par de variáveis [LMI03, [SKAG04]. Assim, a multiinformação pode ser minimizada globalmente por uma série de rotações bidimensionais, alternando-se o par de variáveis envolvido a cada passo. O algoritmo de ICA para dados binários apresentado aqui é análogo a esses métodos e sua construção baseia-se na definição de uma classe de transformações para variáveis aleatórias binárias que permita a decomposição adequada da multi-informação. Como cada passo do método iterativo dá origem a uma transformação local, essa classe de transformações permite a composição de transformações locais a fim de obter-se uma transformação global envolvendo todas as variáveis.

Em um método de ICA, a multi-informação é a grandeza a ser minimizada. Para um vetor $\left(X_{1}, X_{2}, \ldots, X_{n}\right)$ de variáveis aleatórias discretas, a multi-informação é dada por (ver Fórmula 3.8 )

$$
M I\left(X_{1}, X_{2}, \ldots, X_{n}\right)=\sum_{i=1}^{n} H\left(X_{i}\right)-H\left(X_{1}, X_{2}, \ldots, X_{n}\right) .
$$

O método apresentado neste capítulo baseia-se em um tipo de transformação envolvendo pares de variáveis aleatórias discretas que preserva a entropia conjunta $H\left(X_{1}, X_{2}, \ldots, X_{n}\right)$ e permite manipular as entropias condicionais $H\left(X_{i}\right)$, dando 


\begin{tabular}{|c|c|c|}
\hline & $X_{j}=0$ & $X_{j}=1$ \\
\hline$X_{i}=0$ & $p_{00}$ & $p_{01}$ \\
\hline$X_{i}=1$ & $p_{10}$ & $p_{11}$ \\
\hline
\end{tabular}

Tabela 4.1: Tabela de contingência para $P\left(X_{i}, X_{j}\right)$.

\begin{tabular}{|c|c|c|}
\hline & $X_{i} \oplus X_{j}=0$ & $X_{i} \oplus X_{j}=1$ \\
\hline$X_{i}=0$ & $p_{00}$ & $p_{01}$ \\
\hline$X_{i}=1$ & $p_{11}$ & $p_{10}$ \\
\hline
\end{tabular}

Tabela 4.2: Tabela de contingência para $P\left(X_{i}, X_{i} \oplus X_{j}\right)$.

origem a um método de minimização de $M I\left(X_{1}, X_{2}, \ldots, X_{n}\right)$. Essa classe de transformações caracteriza-se por permutações da tabela de contingência do par de variáveis em questão. Por conveniência e visando a exposição futura do método de ICA binária, essa classe de transformações será apresentada por meio de exemplos com variáveis binárias, mas os raciocínio exposto pode ser generalizado para variáveis com mais de dois valores. Considere um vetor de variáveis aleatórias binárias

$$
\mathbf{X}=\left(X_{1}, X_{2}, \ldots, X_{n}\right) \in\{0,1\}^{n} .
$$

Seja $(i, j)$ um par de índices, $1 \leq i<j \leq n$. Tomando-se um par de variáveis $\left(X_{i}, X_{j}\right)$, a distribuição $P\left(X_{i}, X_{j}\right)$ pode ser expressa pela tabela de contingência 4.1 .

Vamos examinar agora a tabela de contingência do par de variáveis $\left(X_{i}, X_{i} \oplus\right.$ $X_{j}$ ) em que $\oplus$ denota a operação ou-exclusivo (os símbolos $p_{00}, p_{01}, p_{10}, p_{11}$ continuam denotando os mesmos valores que na Tabela 4.1).

A transformação $\left(X_{i}, X_{j}\right) \mapsto\left(X_{i}, X_{i} \oplus X_{j}\right)$ corresponde a uma permutação da tabela de contingência (troca de posição dos elementos $p_{10}$ e $p_{11}$ ). Genericamente, vamos nos referir a uma transformação correspondente a uma permutação da tabela de contingência como uma função

$$
\left(Z_{i}, Z_{j}\right)=F\left(X_{i}, X_{j}\right)
$$

mapeando o par de variáveis original $\left(X_{i}, X_{j}\right)$ no par transformado $\left(Z_{i}, Z_{j}\right)$. Quando 
limitada a variáveis binárias, a classe de transformações caracterizada por permutações da tabela de contingência de duas variáveis equivale à classe das funções Booleanas bijetoras $F:\{0,1\}^{2} \mapsto\{0,1\}^{2}$. Obviamente, o tipo de transformação apresentada é reversível, pois podemos permutar novamente posições da tabela e obter a tabela original. No caso do exemplos apresentado, a reversibilidade também pode ser verificada pelo exame da identidade

$$
X_{i} \oplus X_{i} \oplus X_{j}=X_{j}
$$

Quando generalizada para variáveis de cardinalidade maior que 2, a relação entre o espaço dos atributos originais $\left(\mathcal{X}_{i}, \mathcal{X}_{j}\right)$ e o espaço dos atributos transformados $\left(\mathcal{Z}_{i}, \mathcal{Z}_{j}\right)$ torna-se mais complicada do que no caso de variáveis binárias, mas é preservada a cardinalidade das variáveis, ou seja:

$$
\left|\mathcal{Z}_{i}\right|=\left|\mathcal{X}_{i}\right|
$$

e

$$
\left|\mathcal{Z}_{j}\right|=\left|\mathcal{X}_{j}\right|
$$

Isso ocorre porque apenas são permitidas trocas entre posições da tabela de contingência, mas o formato da tabela (número de linhas e colunas) não é alterado.

Agora vamos mostrar que uma transformação de permutação não altera a entropia conjunta, isto é

$$
H\left(X_{i}, X_{j}\right)=H\left(Z_{i}, Z_{j}\right)
$$

Usando a definição de entropia conjunta, nós temos:

$$
H\left(X_{i}, X_{j}\right)=-\sum_{x_{i} \in \mathcal{X}_{i}} \sum_{x_{j} \in \mathcal{X}_{j}} P_{X_{i} X_{j}}\left(x_{i}, x_{j}\right) \log P_{X_{i} X_{j}}\left(x_{i}, x_{j}\right)
$$

e

$$
H\left(Z_{i}, Z_{j}\right)=-\sum_{z_{i} \in \mathbf{Z}_{i}} \sum_{z_{j} \in \mathbf{Z}_{j}} P_{Z_{i} Z_{j}}\left(z_{i}, z_{j}\right) \log P_{Z_{i} Z_{j}}\left(z_{i}, z_{j}\right)
$$

Nas expressões acima foi adicionado o índice subscrito a cada medida de probabilidade para evidenciar quais as variáveis envolvidas. A cada elemento $\left(x_{i}, x_{j}\right)$ do espaço amostral do par $\left(X_{i}, X_{j}\right)$ corresponde um único elemento $\left(z_{i}, z_{j}\right)$ do 
espaço amostral de $\left(Z_{i}, Z_{j}\right)$, pois a relação entre eles é estabelecida pela permutação da tabela de contingência. Devido à essa correspondência biunívoca entre elementos de $\left(\mathcal{X}_{i}, \mathcal{X}_{j}\right)$ e de $\left(\mathcal{Z}_{i}, \mathcal{Z}_{j}\right)$, nós temos que:

$$
P_{Z_{i} Z_{j}}\left(z_{i}, z_{j}\right)=P_{X_{i} X_{j}}\left(x_{i}, x_{j}\right)
$$

Logo, devido à relação (4.5) as duas expressões (4.3) e (4.4) são equivalentes e

$$
H\left(X_{i}, X_{j}\right)=H\left(Z_{i}, Z_{j}\right)
$$

Quando esse tipo de transformação é aplicada a um par de elementos de um vetor binário $n$-dimensional, $n>2$, observa-se que a entropia conjunta total também é preservada. Denote-se por $\mathbf{Z}$ o seguinte vetor aleatório:

$$
\mathbf{Z}=\left(X_{1}, \ldots, X_{i-1}, Z_{i}, X_{i+1}, \ldots, X_{j-1}, Z_{j}, X_{j+1} \ldots, X_{n}\right)
$$

obtido a partir de $\mathbf{X}$ pela substituição de $\left(X_{i}, X_{j}\right)$ por $\left(Z_{i}, Z_{j}\right)$. De forma geral, denotaremos esse tipo de transformação por $\mathbf{Z}=F(\mathbf{X})$. Sejam $\mathbf{x}$ uma realização de $\mathbf{X}$ e $\mathbf{z}$ uma realização de $\mathbf{Z}$. $\mathbf{O}$ argumento da correspondência biunívoca entre $\left(z_{i}, z_{j}\right)$ e $\left(x_{i}, x_{j}\right)$ pode ser usado novamente para mostrar que:

$$
\begin{gathered}
P_{\mathbf{Z}}\left(x_{1}, \ldots, x_{i-1}, z_{i}, x_{i+1}, \ldots, x_{j-1}, z_{j}, x_{j+1} \ldots, x_{n}\right)= \\
P_{\mathbf{X}}\left(x_{1}, \ldots, x_{i-1}, x_{i}, x_{i+1}, \ldots, x_{j-1}, x_{j}, x_{j+1} \ldots, x_{n}\right) .
\end{gathered}
$$

De forma mais compacta, podemos escrever:

$$
P_{\mathbf{Z}}(\mathbf{z})=P_{\mathbf{X}}(\mathbf{x})
$$

Logo:

$$
\sum_{\mathbf{z}} P_{\mathbf{Z}}(\mathbf{z}) \log P_{\mathbf{Z}}(\mathbf{z})=\sum_{\mathbf{x}} P_{\mathbf{X}}(\mathbf{x}) \log P_{\mathbf{X}}(\mathbf{x})
$$

e

$$
H(\mathbf{Z})=H(\mathbf{X})
$$

Outro modo de compreender o resultado acima decorre da observação que a entro- 
pia conjunta é a média da quantidade - $\log p_{k}$ para uma distribuição determinada por valores $p_{k}\left(p_{k}>0\right.$ para todo $\left.k ; \sum_{k} p_{k}=1\right)$. A transformação de permutação não altera os valores de $p_{k}$, apenas muda os símbolos associados a cada $p_{k}$ (no exemplo, tais símbolos são realizações de vetores binários), portanto a entropia conjunta não se altera. No entanto, a permutação altera a maneira pela qual somamos os valores $p_{k}$ para obter as distribuições marginais das variáveis. Logo, apesar de preservar a entropia conjunta, esse tipo de transformação pode alterar as entropias marginais das variáveis resultantes da transformação (em relação às variáveis originais). Voltando ao exemplo anterior envolvendo um par de variáveis binárias, com

$$
\left(Z_{i}, Z_{j}\right)=\left(X_{i}, X_{i} \oplus X_{j}\right)
$$

as entropias marginais são dadas por:

$$
H\left(X_{j}\right)=\xi\left(p_{00}+p_{10}\right)+\xi\left(p_{01}+p_{11}\right)
$$

e

$$
H\left(Z_{j}\right)=\xi\left(p_{00}+p_{11}\right)+\xi\left(p_{01}+p_{10}\right)
$$

em que $\xi(w)=-w \log w$. Conclui-se que as entropias marginais $H\left(X_{j}\right)$ e $H\left(Z_{j}\right)$ serão diferentes, a menos que $p_{11}=p_{10}$. Tal conclusão reflete-se no cálculo da multi-informação de um vetor de variáveis. Observando-se que a multiinformação $M I(\mathbf{Z})$ após a transformação é dada por:

$$
\begin{aligned}
M I(\mathbf{Z})= & H\left(Z_{i}\right)+H\left(Z_{j}\right) \\
& +\sum_{k \neq i, j} H\left(X_{k}\right)-H(\mathbf{Z})
\end{aligned}
$$

e subtraindo-se a Equação (4.2) de (4.10) e aplicando-se a relação 4.9), obtêm-se:

$$
M I(\mathbf{Z})-M I(\mathbf{X})=H\left(Z_{i}\right)+H\left(Z_{j}\right)-H\left(X_{i}\right)-H\left(X_{j}\right)
$$

Lembrando que a multi-informação reduz-se a informação mútua quando consi- 
deradas apenas duas variáveis, isto é (ver expressões 3.5 e 3.8)

$$
M I\left(X_{i}, X_{j}\right)=I\left(X_{i} ; X_{j}\right)
$$

podemos escrever:

$$
I\left(Z_{i} ; Z_{j}\right)-I\left(X_{i} ; X_{j}\right)=H\left(Z_{i}\right)+H\left(Z_{j}\right)-H\left(X_{i}\right)-H\left(X_{j}\right) .
$$

As expressões (4.11) e (4.12) valem genericamente para transformações caracterizadas por permutações numa tabela de contingência contendo duas variáveis aleatórias discretas (não necessariamente binárias). Para o exemplo envolvendo variáveis binárias utilizado ao longo do capítulo, as expressões podem ser simplificadas ao notarmos que $Z_{i}=X_{i}$ :

$$
\begin{gathered}
M I(\mathbf{Z})-M I(\mathbf{X})=H\left(Z_{j}\right)-H\left(X_{j}\right) \\
I\left(Z_{i} ; Z_{j}\right)-I\left(X_{i} ; X_{j}\right)=H\left(Z_{j}\right)-H\left(X_{j}\right) .
\end{gathered}
$$

A expressão (4.13) mostra que é possível reduzir a multi-informação de um vetor de variáveis binárias se $H\left(Z_{j}\right)-H\left(X_{j}\right)<0$. Tal observação é a base do novo algoritmo de ICA apresentado neste capítulo. Até agora utilizamos o exemplo em que a permutação da tabela de contingência das variáveis binárias corresponde à função XOR, ou seja $Z_{j}=X_{i} \oplus X_{j}$. Antes de enunciar o novo algoritmo de ICA, vamos mostrar que a função XOR é a única função correspondente a permutações que precisa ser considerada nesse contexto.

O espaço amostral associado a $\left(X_{i}, X_{j}\right) \in\{0,1\}^{2}$ tem quatro elementos:

$$
\{(0,0),(0,1),(1,0),(1,1)\} \text {. }
$$

Portanto, a tabela de contingência de $\left(X_{i}, X_{j}\right)$ tem 4 elementos e o número total de transformações correspondentes a permutações dessa tabela é igual ao número de permutações possíveis para um conjunto de 4 elementos, ou seja, 4 ! $=24$ transformações possíveis. No entanto, somente nos interessam as transformações que resultam em alteração da soma das entropias marginais $H\left(Z_{i}\right)+H\left(Z_{j}\right)$, pois só assim a multi-informação será alterada, como pode ser depreendido da Equa- 
ção (4.12). Algumas permutações conservam o valor dessa soma de entropias. Por exemplo, simplesmente trocar a posição das variáveis $X_{i}$ e $X_{j}$

$$
\left(Z_{i}, Z_{j}\right)=\left(X_{j}, X_{i}\right)
$$

implica

$$
H\left(Z_{i}\right)=H\left(X_{j}\right)
$$

$\mathrm{e}$

$$
H\left(Z_{j}\right)=H\left(X_{i}\right)
$$

Obviamente, segue que

$$
H\left(Z_{i}\right)+H\left(Z_{j}\right)=H\left(X_{i}\right)+H\left(X_{j}\right)
$$

Essa permutação corresponde à transposição da tabela de contingência, ou seja, transformar linhas em colunas da tabela.

Outro tipo de transformação que mantém a soma das entropias marginais é obtido pela negação de uma ou duas variáveis. Esse fato decorre da fórmula de entropia para uma variável binária.

$$
H(X)=-\sum_{x \in\{0,1\}} P(X=x) \log P(X=x)
$$

Dado que $P(X=0)=P(\bar{X}=1)$ e $P(X=1)=P(\bar{X}=0)$, nós temos que $H(X)=H(\bar{X})$. Tal permutação corresponde a trocar as linhas da tabela de contingência entre si (ou o mesmo para as colunas).

Da discussão acima, decorre que para um par de variáveis binárias $(A, B)$, podemos compor as operações elementares acima (troca de posição entre as variáveis e negação) para definir 8 transformações que preservam a soma das entropias marginais, representadas aqui pelo par de variáveis resultante da transformação (note que o primeiro par se refere à transformação identidade):

$$
\{(A, B),(\bar{A}, B),(A, \bar{B}),(\bar{A}, \bar{B}),(B, A),(B, \bar{A}),(\bar{B}, A),(\bar{B}, \bar{A})\} .
$$

Segue que, embora existam 24 transformações correspondentes a permutações, 
existem no máximo $24 / 8=3$ valores diferentes para a soma das entropias marginais.

O primeiro valor é dado pelas variáveis originais: $H\left(X_{i}\right)+H\left(X_{j}\right)$. Os outros dois correspondem aos mapeamentos

$$
\left(Z_{i}, Z_{j}\right)=\left(X_{i}, X_{i} \oplus X_{j}\right)
$$

$\mathrm{e}$

$$
\left(Z_{i}, Z_{j}\right)=\left(X_{i} \oplus X_{j}, X_{j}\right)
$$

em que $\oplus$ expressa a operação ou-exclusivo (XOR). A Tabela 4.3 ilustra como esses mapeamentos se relacionam com permutações dos elementos em $\{0,1\}^{2}$.

\begin{tabular}{|c|c|c|}
\hline$X_{i}, X_{j}$ & $X_{i}, X_{i} \oplus X_{j}$ & $X_{i} \oplus X_{j}, X_{j}$ \\
\hline 0,0 & 0,0 & 0,0 \\
\hline 0,1 & 0,1 & $\mathbf{1}, \mathbf{1}$ \\
\hline 1,0 & $\mathbf{1}, \mathbf{1}$ & 1,0 \\
\hline 1,1 & $\mathbf{1}, \mathbf{0}$ & $\mathbf{0}, \mathbf{1}$ \\
\hline
\end{tabular}

Tabela 4.3: As duas transformações de permutação que alteram a informação mútua. Para cada valor possível do par de variáveis originais, a tabela mostra o valor do par transformado. Os valores permutados são mostrados em negrito.

A variável resultante da aplicação da operação XOR tem distribuição marginal diferente das variáveis aleatórias originais, como pode ser concluído a partir das seguintes equações:

$$
\begin{aligned}
& P\left(X_{i} \oplus X_{j}=0\right)=P\left(X_{i}=0, X_{j}=0\right)+P\left(X_{i}=1, X_{j}=1\right) \\
& P\left(X_{i} \oplus X_{j}=1\right)=P\left(X_{i}=0, X_{j}=1\right)+P\left(X_{i}=1, X_{j}=0\right)
\end{aligned}
$$

e portanto a entropia $H\left(X_{i} \oplus X_{j}\right)$ também é diferente de $H\left(X_{i}\right)$ e $H\left(X_{j}\right)$ (salvo uma coincidência numérica). Esse resultado sugere o seguinte procedimento para minimizar a multi-informação de um vetor de variáveis binárias: escolhe-se um par de índices $(i, j)$, calculando-se os valores de $H\left(X_{i}\right), H\left(X_{j}\right), H\left(X_{i} \oplus X_{j}\right)$. Seja

$$
m=\arg \max _{i, j}\left(H\left(X_{i}\right), H\left(X_{j}\right)\right)
$$


caso $H\left(X_{i} \oplus X_{j}\right)<H\left(X_{m}\right)$ então a multi-informação pode ser reduzida pela substituição de $X_{m}$ por $X_{i} \oplus X_{j}$.

Seja a distribuição $P\left(X_{i}, X_{j}\right)$ determinada por quatro valores $p_{a}, p_{b}, p_{c}, p_{d}$, cada valor determinando a probabilidade $P\left(X_{i}=x_{i}, X_{j}=x_{j}\right)$ associada a cada $\operatorname{par}\left(x_{i}, x_{j}\right) \in\{0,1\}^{2}$, sendo:

$$
0 \leq p_{a} \leq p_{b} \leq p_{c} \leq p_{d} \leq 1
$$

e

$$
p_{a}+p_{b}+p_{c}+p_{d}=1 \text {. }
$$

Para que o valor mínimo de $H\left(X_{i}\right)+H\left(X_{j}\right)$ seja atingido é necessário que as duas distribuições marginais $P\left(X_{i}\right)$ e $P\left(X_{j}\right)$ tenham a maior concentração possível. Isso ocorre quando o maior e o menor parâmetros encontram-se na diagonal da tabela de contingência das duas variáveis como, por exemplo, na Tabela 4.4. Assim, o cálculo das distribuições marginais envolve a soma dos dois maiores valores $\left(p_{c}+p_{d}\right)$ para a primeira variável e a soma do maior valor com o terceiro maior $\left(p_{b}+p_{d}\right)$ para a segunda variável.

\begin{tabular}{|c|c|c|}
\hline & $X_{i}=0$ & $X_{i}=1$ \\
\hline$X_{j}=0$ & $p_{a}$ & $p_{b}$ \\
\hline$X_{j}=1$ & $p_{c}$ & $p_{d}$ \\
\hline
\end{tabular}

Tabela 4.4: Tabela de contingência para $P\left(X_{s}, X_{t}\right)$

Para aplicar esse procedimento iterativamente é necessário combinar transformações sucessivas, i.e. calcular a função XOR sobre pares de variáveis já transformadas. Nessa situação a função XOR é generalizada pela função paridade (PAR). Seja um conjunto $W$ de variáveis binárias, expresso por

$$
W=\left\{w_{1}, w_{2}, \ldots, w_{K}\right\}, w_{k} \in\{0,1\}
$$


a paridade do conjunto é definida por:

$$
\operatorname{PAR}(W)= \begin{cases}0 & \text { se } \sum_{k=1}^{K} w_{k} \text { é par, } \\ 1 & \text { se } \sum_{k=1}^{K} w_{k} \text { é ímpar. }\end{cases}
$$

A função XOR é equivalente à função PAR para duas variáveis. Para nossos propósitos, será útil estabelecer um paralelo entre transformações aplicadas em vetores binários e operações entre subconjuntos dos elementos desse vetor. Por exemplo, a substituição do elemento $X_{i}$ de um vetor por $X_{i} \oplus X_{j}$ corresponde a união entre os conjuntos $\left\{X_{i}\right\}$ e $\left\{X_{j}\right\}$, originando $\left\{X_{i}, X_{j}\right\}$. Dados dois subconjuntos formados por elementos de $\mathrm{X}$,

$$
W_{k}, W_{l} \subseteq\left\{X_{1}, X_{2}, \ldots, X_{n}\right\}
$$

nós temos:

$$
\operatorname{PAR}\left(W_{k}\right) \oplus \operatorname{PAR}\left(W_{l}\right)=\operatorname{PAR}\left(W_{k} \cup W_{l} \backslash W_{k} \cap W_{l}\right)
$$

em que \denota subtração entre conjuntos. As variáveis no conjunto intersecção $W_{k} \cap W_{l}$ são removidas do conjunto final porque termos que aparecem um número par de vezes durante o cálculo da paridade não afetam o valor da função. A expressão 4.17) mostra que a aplicação iterativa de transformações de duas variáveis levam à formação de grupos de variáveis binárias. Cada variável pode pertencer a mais de um grupo simultaneamente. Aqui, evitou-se de maneira proposital chamar esses grupos de agrupamentos ou conglomerados (clusters) pois esses dois termos sinônimos têm significados precisos dentro da análise de agrupamentos. A análise de agrupamentos permite interpretar os seus resultados em termos de medidas de pertinência a conjuntos e/ou modelos probabilísticos, o que não é o caso com o presente método (a palavra grupo também está sendo usada no seu sentido comum de coleção de coisas e não no sentido que tem na álgebra, por exemplo).

Um modo conveniente de representar os grupos de variáveis que definem a transformação

$$
\mathbf{Z}=F(\mathbf{X})
$$


é por meio de uma matriz de cálculo de paridade. Seja $W$ um subconjunto dos elementos de $\mathbf{X} \in\{0,1\}^{n}$. Pode-se representar o conjunto $W$ por um vetor índice $\mathbf{w} \in\{0,1\}^{n}$, de tal modo que

$$
w_{k}=1 \Longleftrightarrow X_{k} \in W
$$

A paridade sobre os elementos de $W$ pode ser computada por meio de um produto escalar $\mathbf{w}^{T} \mathbf{X}$, sendo o cálculo efetuado utilizando-se aritmética módulo-2 (i.e., $0+1=1,1+1=0$, etc) [Mac03, cap. 1]. Desse ponto em diante, todas as operações envolvendo vetores e matrizes binários utilizarão aritmética módulo2, a menos que o texto aponte explicitamente o contrário. Por exemplo, com $\mathbf{X} \in\{0,1\}^{5}$ e $W=\left\{X_{2}, X_{4}, X_{5}\right\}$, teríamos

$$
\mathbf{w}=(0,1,0,1,1)^{T}
$$

Expandindo esse conceito, a transformação $\mathbf{Z}=F(\mathbf{X})$ requer a multiplicação de $\mathrm{X}$ por uma matriz binária $n \mathrm{x} n$, sendo escrita como:

$$
\mathbf{Z}=\mathbf{W X}
$$

em que $\mathbf{Z}, \mathbf{X} \in\{0,1\}^{n}$, W é uma matriz $n \times n$ com elementos binários: $w_{i j} \in$ $\{0,1\}$, denominada matriz de separação. Cada linha de $\mathbf{W}$ corresponde a um dos grupos de variáveis que definem a transformação. Dados dois grupos distintos de variáveis, representados pelos conjuntos $W_{k}$ e $W_{l}$, a combinação dos grupos (Equação 4.17) pode ser calculada pela soma dos respectivos vetores:

$$
\operatorname{PAR}\left(W_{k}\right) \oplus \operatorname{PAR}\left(W_{l}\right)=\left(\mathbf{w}_{k}+\mathbf{w}_{l}\right)^{T} \mathbf{X}
$$

A Equação 4.18 mostra que a transformação utilizada no procedimento de ICA binária pode ser concebida como uma mudança de coordenadas em $\{0,1\}^{n}$.

Agora estamos em condições de enunciar o novo algoritmo para análise de componentes independentes de variáveis binárias (Algoritmo1). O algoritmo re- 
cebe como entrada uma série de realizações

$$
\mathbf{x}^{(1)}, \mathbf{x}^{(2)}, \ldots, \mathbf{x}^{(L)}
$$

de um vetor aleatório binário $\mathbf{X} \in\{0,1\}^{n}$. Para que o algoritmo funcione com um nível aceitável de precisão estatística é preciso ter $L>>n$. O algoritmo produz a matriz de separação $W$ e uma série

$$
\mathbf{z}^{(1)}, \mathbf{z}^{(2)}, \ldots, \mathbf{z}^{(L)}
$$

de vetores resultantes da transformação, ou seja,

$$
\mathbf{z}^{(i)}=\mathbf{W} \mathbf{x}^{(i)}, \mathbf{z}^{(i)} \in\{0,1\}^{n}
$$

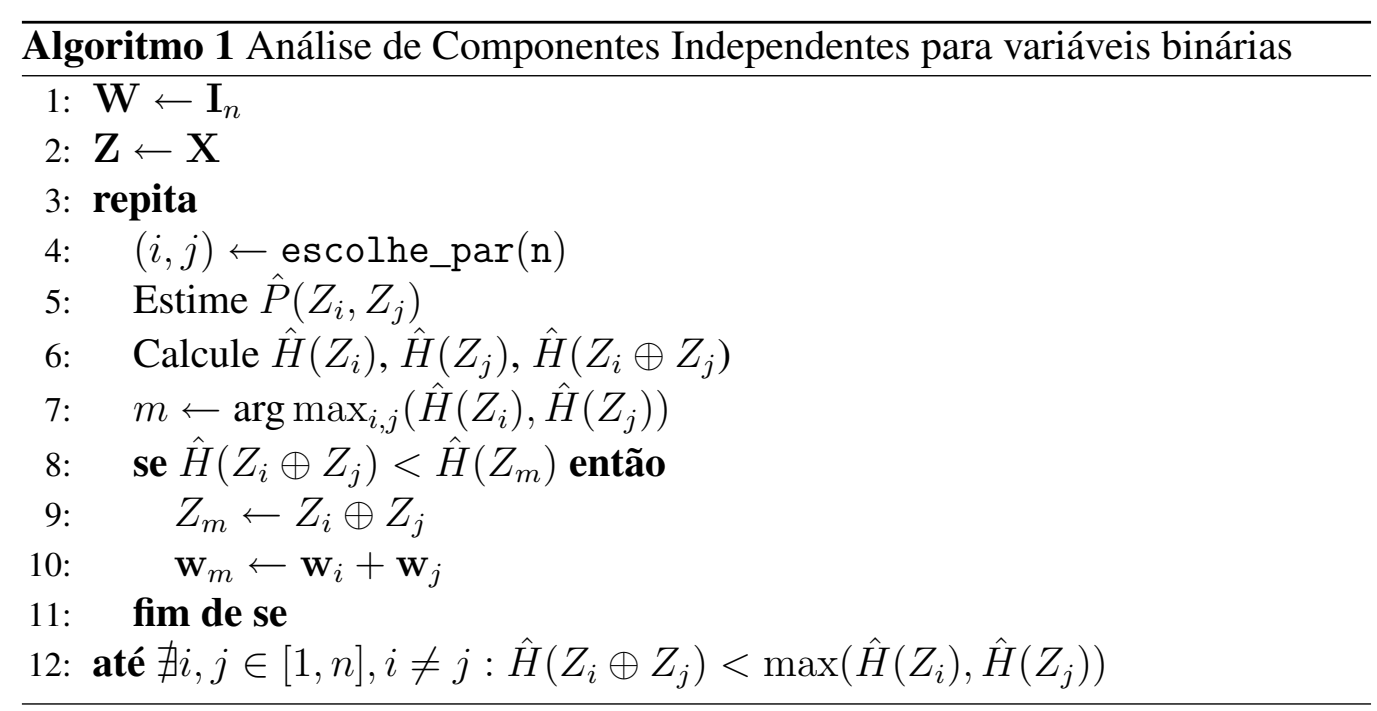

Inicialmente, a matriz de separação $\mathrm{W}$ é igual à matriz identidade, logo te$\operatorname{mos} \mathbf{Z}=\mathbf{X}$ e os grupos de variáveis são triviais, cada grupo contendo uma única variável distinta $X_{n}$. A cada iteração, um par de índices distintos é selecionado pelo procedimento escolhe_par (essa escolha pode ser aleatória ou o conjunto de índices pode ser percorrido de maneira sistemática). A distribuição $\hat{P}\left(Z_{i}, Z_{j}\right)$ é estimada, usando-se um estimador do tipo histograma. Em seguida as entropias marginais relevantes são calculadas, utilizando-se a estimativa $\hat{P}\left(Z_{i}, Z_{j}\right)$. O algoritmo então decide se deve aplicar uma transformação de par de variáveis 
nessa iteração, o que ocorre caso a maior entropia marginal $\max \left(\hat{H}\left(Z_{i}\right), \hat{H}\left(Z_{j}\right)\right)$ seja maior que $\hat{H}\left(Z_{i} \oplus Z_{j}\right)$, isto é, caso uma das variáveis possa ser substituída por $Z_{i} \oplus Z_{j}$, resultando em diminuição da entropia marginal. Nesse caso ou algoritmo aplica a transformação adequada aos dados e atualiza a matriz $\mathbf{W}$. A atualização da matriz consiste em trocar a linha correspondente à variável substituída pela soma (módulo-2) das duas linhas da envolvidas nessa iteração, dado pela expressão $\mathbf{w}_{i}+\mathbf{w}_{j}$. Caso contrário, nenhuma transformação á aplicada nessa iteração e o algoritmo simplesmente continua com uma nova iteração, com outro par de índices. O processo é repetido até que não existam mais índices $(i, j)$ tal que a transformação de par de variáveis seja possível, ou seja, isso ocorre quando qualquer transformação de par de variáveis resultaria em aumento das entropias marginais.

O processo de aplicar uma transformação pode ser entendido como uma multiplicação de matrizes. Suponha-se que na $k$-ésima iteração a matriz de separação seja $\mathbf{W}^{(k)}$ e a transformação de par de variáveis aplicada corresponda a $Z_{i} \leftarrow Z_{i} \oplus Z_{j}$. Podemos escrever

$$
\mathbf{W}^{(k+1)}=\mathbf{B}^{(k)} \mathbf{W}^{(k)},
$$

em que $\mathbf{B}^{(k)}$ é uma matriz binária $n \times n$ cujos elementos da diagonal principal são todos iguais a 1 , sendo os outros elementos iguais a zero, exceto pelo elemento $b_{i j}=1$. Um exemplo é dado a seguir, em que $n=4$ e a matriz $\mathbf{B}^{(k)}$ corresponde à transformação $Z_{3} \leftarrow Z_{1} \oplus Z_{3}$

$$
\mathbf{B}^{(k)}=\left(\begin{array}{cccc}
1 & 0 & 0 & 0 \\
0 & 1 & 0 & 0 \\
1 & 0 & 1 & 0 \\
0 & 0 & 0 & 1
\end{array}\right)
$$

Seja $\mathbf{M}$ uma matriz binária $n \mathbf{x} n$, denote-se as linhas de $\mathbf{M}$ por $\mathbf{m}_{1}, \mathbf{m}_{2}, \ldots, \mathbf{m}_{n}$. O efeito de multiplicar $\mathbf{M}$ por $\mathbf{B}^{(k)}$ pela esquerda equivale a criar uma nova matriz $\mathbf{M}^{\prime}=\mathbf{B}^{(k)} \mathbf{M}$ cujas linhas $\mathbf{m}_{k}^{\prime}$ são iguais às de $\mathbf{M}$, exceto pela $i$-ésima linha, igual 
$\mathrm{a} \mathbf{m}_{i}+\mathbf{m}_{j}$ :

$$
\begin{gathered}
\forall k \in\{1,2, \ldots, i-1, i+1, \ldots, n\}: \mathbf{m}_{k}^{\prime}=\mathbf{m}_{k} \\
\mathbf{m}_{i}^{\prime}=\mathbf{m}_{i}+\mathbf{m}_{j} .
\end{gathered}
$$

Se repetirmos essa operação, teremos $\mathbf{M}^{\prime \prime}=\mathbf{B}^{(k)} \mathbf{M}^{\prime}$ e, portanto:

$$
\begin{gathered}
\forall k \in\{1,2, \ldots, i-1, i+1, \ldots, n\}: \mathbf{m}^{\prime \prime}{ }_{k}=\mathbf{m}_{k}^{\prime}=\mathbf{m}_{k} \\
\mathbf{m}_{i}^{\prime \prime}=\mathbf{m}_{i}^{\prime}+\mathbf{m}_{j}^{\prime} \\
=\mathbf{m}_{i}+\mathbf{m}_{j}+\mathbf{m}_{j} .
\end{gathered}
$$

Devido ao uso de aritmética módulo-2, temos:

$$
\mathbf{m}_{j}+\mathbf{m}_{j}=\mathbf{0} .
$$

Das equações (4.22) e (4.24) segue que:

$$
\mathbf{m}_{i}^{\prime \prime}=\mathbf{m}_{i}
$$

Logo,

$$
\mathbf{M}^{\prime \prime}=\mathbf{B}^{(k)} \mathbf{B}^{(k)} \mathbf{M}=\mathbf{M}
$$

o que mostra que

$$
\mathbf{B}^{(k)} \mathbf{B}^{(k)}=I_{n},
$$

isto é, a matriz $\mathbf{B}^{(k)}$ é sua própria inversa.

Lembrando que no início do Algoritmo 1 temos $\mathbf{W}^{(1)}=\mathbf{I}_{n}$, podemos expandir $\mathbf{W}^{(k)}$ como:

$$
\mathbf{W}^{(k)}=\mathbf{B}^{(k-1)} \mathbf{B}^{(k-2)} \ldots \mathbf{B}^{(2)} \mathbf{B}^{(1)} .
$$

Do mesmo modo, podemos construir uma matriz $\mathbf{A}^{(k)}=\left[\mathbf{W}^{(k)}\right]^{-1}$ :

$$
\mathbf{A}^{(k)}=\mathbf{B}^{(1)} \mathbf{B}^{(2)} \ldots \mathbf{B}^{(k-2)} \mathbf{B}^{(k-1)}
$$


aproveitando o fato que $\mathbf{B}^{(k)} \mathbf{B}^{(k)}=I_{n}$. A matriz $\mathbf{A}$ é chamada matriz de mistura no jargão de ICA, pois realiza a mistura das fontes para a síntese dos dados observados:

$$
\mathbf{x}=\mathbf{A z}
$$

\subsection{Convergência, complexidade e qualidade da solução}

O Algoritmo 1 busca minimizar a multi-informação $M I(\mathbf{Z})$ das variáveis latentes obtidas por meio de uma transformação $\mathbf{Z}=\mathbf{W X}$, em que $\mathbf{W}$ é uma matriz binária $n \times n n$, construída de tal modo que $H(\mathbf{Z})=H(\mathbf{X})$. Foi demonstrado anteriormente que minimizar $M I(\mathbf{Z})$ nessas condições equivale a minimizar $\sum_{i=1}^{n} H\left(Z_{i}\right)$. Cada uma das possíveis matrizes $\mathbf{W}$ pode ser associada a um único valor $\sum_{i=1}^{n} H\left(Z_{i}\right)$, obtido quando essa matriz é usada para a transformação. Em cada iteração em que o Algoritmo 1 atualiza a matriz W, a somatória $\sum_{i=1}^{n} H\left(Z_{i}\right)$ decai, de modo que essa grandeza segue trajetória estritamente decrescente. Logo, não há repetição de matrizes, nunca a atualização da matriz $\mathbf{W}$ fará com que essa matriz assuma um valor já obtido em iteração anterior. Como o número de possíveis matrizes binárias $n \times n$ é finito e a atualização realizada pelo algoritmo resulta sempre em uma matriz inédita, segue que em algum momento o critério de parada do algoritmo será atingido, não sendo mais possível criar uma nova matriz $\mathbf{W}$ tal que a quantidade $\sum_{i=1}^{n} H\left(Z_{i}\right)$ diminua.

Para estudar a velocidade de convergência do algoritmo, vamos examinar o número de possíveis matrizes $\mathbf{W}$ que podem ser geradas pelo algoritmo. Em primeiro lugar, pode-se constatar que a solução do problema não é única. A quantidade minimizada pelo algoritmo, $\sum_{i=1}^{n} H\left(Z_{i}\right)$, é indiferente à ordem de apresentação das variáveis $Z_{i}$. Logo, sendo $\mathbf{W}$ uma matriz que minimiza $\sum_{i=1}^{n} H\left(Z_{i}\right)$ e sendo $\mathbf{P}$ uma matriz de permutação (uma matriz binária em que cada linha e cada coluna contêm apenas um elemento diferente de zero), a matriz PW também é solução do problema da ICA binária. Como, para o Algoritmo 11, o valor de $\sum_{i=1}^{n} H\left(Z_{i}\right)$ é estritamente decrescente em função do número de atualizações realizadas em $\mathbf{W}$, segue que o algoritmo nunca produz as permutações de linha de uma matriz $\mathbf{W}$ já examinada anteriormente, do contrário o valor de $\sum_{i=1}^{n} H\left(Z_{i}\right)$ se manteria o mesmo ou aumentaria. Um segundo ponto a ser observado é que as linhas de $\mathbf{W}$ são capazes de gerar todo o espaço $\{0,1\}^{n}$, não há linhas linear- 
mente dependentes entre si. Inicialmente, a matriz $\mathbf{W}$ é igual a $\mathbf{I}_{n}$ e suas linhas formam uma base trivial para $\{0,1\}^{n}$. Ao longo da execução do algoritmo, cada atualização de $\mathbf{W}$ somente substitui uma linha $\mathbf{w}_{i}$ da matriz por esse mesma linha somada a outra linha $\mathbf{w}_{j}$. Supondo que $\mathbf{w}_{i}$ e $\mathbf{w}_{j}$ sejam linearmente independentes com relação ao restante das linhas da matriz, então $\mathbf{w}_{i}+\mathbf{w}_{j}$ deve ser linearmente independente também, do contrário poderíamos criar representações lineares para $\mathbf{w}_{i}$ e $\mathbf{w}_{j}$ a partir da representação linear existente para $\mathbf{w}_{i}+\mathbf{w}_{j}$ em termos do restante das linhas da matriz. Desse modo, em cada iteração do Algoritmo 1, a matriz $\mathbf{W}$ conserva-se capaz de gerar todo o espaço $\{0,1\}^{n}$ com suas linhas.

Agora podemos estimar o número de matrizes binárias $n \times n$ que podem ser geradas pelo algoritmo. O número de possíveis linhas distintas e não-nulas da matriz é $2^{n}-1$. Vamos chamar de $C$ esse conjunto das linhas. Logo, $|C|=$ $2^{n}-1$. Para formar uma matriz $\mathbf{W}$, vamos escolher sequencialmente $n$ elementos de $C$. No entanto, a cada passo devemos subtrair do conjunto de linhas que podem ser escolhidas aquelas que sejam linearmente dependentes com relação as linhas previamente escolhidas. Isto é:

- No primeiro passo, escolhemos uma linha $\mathbf{w}_{1}$ entre $|C|$ linhas possíveis.

- No segundo passo, temos de subtrair a linha escolhida anteriormente. A segunda linha $\mathbf{w}_{2}$ é escolhida de um conjunto de $|C|-1$ linhas.

- No terceiro passo, precisamos subtrair as duas linhas anteriormente escolhidas e também aquela formada pela combinação delas, isto é $\mathbf{w}_{1}+\mathbf{w}_{2}$. Então $\mathbf{w}_{3}$ é escolhida a partir de um conjunto contendo $|C|-3$ linhas.

O processo continua até $\mathbf{w}_{n}$.

Se $k$ linhas já tiverem sido selecionadas em passos anteriores, o número de linhas distintas e não nulas que podem ser formadas por combinação linear dessas $k$ linhas é dado por $2^{k}-1$. Cada linha pode participar da combinação linear com o respectivo coeficiente igual a 0 ou igual a 1 , o que resulta em $2^{k}$ combinações possíveis; esse numero deve ser decrementado em uma unidade devido ao vetor nulo, resultando no valor anunciado. Logo, o número de matrizes binárias $n \times n$ distintas e capazes de gerar todo o espaço $\{0,1\}^{n}$ é dado por:

$$
\mid \text { Matrizes }|=| C \mid \cdot(|C|-1) \cdot(|C|-3) \cdot(|C|-7) \cdot \ldots \cdot\left(|C|-2^{n-1}+1\right) .
$$


Substituindo $|C|$ por $2^{n}-1$ :

$$
\begin{aligned}
\mid \text { Matrizes } \mid & =\left(2^{n}-1\right) \cdot\left(2^{n}-2\right) \cdot\left(2^{n}-4\right) \ldots \cdot\left(2^{n}-2^{n-1}\right) \\
& =\prod_{i=0}^{n-1}\left(2^{n}-2^{i}\right) .
\end{aligned}
$$

Pelo motivo exposto anteriormente, devemos descontar as permutações de linhas para obter o número de matrizes que o Algoritmo 1 é capaz de gerar ao longo de sua execução:

$$
\mid \text { Matrizes alg. ICA } \mid=\frac{\prod_{i=0}^{n-1}\left(2^{n}-2^{i}\right)}{n !} .
$$

A equação 4.32 estabelece um teto máximo para o número de iterações necessárias para a convergência do algoritmo. Na prática, foi observado que a convergência acontece em um número de iterações bem menor. Note-se que o critério de parada do Algoritmo 1 exige que todos os pares de índices $(i, j), i \neq j$ sejam avaliados quanto a possibilidade de se aplicar uma nova transformação. Por isso, o número mínimo de iterações que o algoritmo realiza é $O\left(n^{2}\right)$.

O produto (número de iterações)x(complexidade de iteração) determina a complexidade total do Algoritmo 1. Dentro de cada iteração, estimar $\hat{P}\left(X_{i}, X_{j}\right)$ com um estimador de histograma e atualizar a variável latente $Z_{m}$ são operações que exigem percorrer todo o conjunto de dados, sendo, portanto, operações $O(L)$, em que $L$ é o tamanho do conjunto de treinamento. Atualizar a matriz $\mathbf{W}$ é uma operação $O(n)$, pois envolve alterar apenas uma de suas linhas. A escolha de índices $(i, j)$ e o cálculo das entropias são operações de tempo constante. A verificação do critério de parada pode ser feita mantendo-se uma contagem do número de iterações consecutivas em que o algoritmo não foi capaz de atualizar W. A cada vez que o algoritmo aplica uma atualização a contagem é reiniciada. Assim, a execução do algoritmo pode ser terminada quando a contagem atingir o número total de pares $(i, j)$, indicando que todos os pares foram testados consecutivamente, sem que a multi-informação pudesse ser minimizada. Manter essa contagem é uma operação de tempo constante. Logo, a execução de cada iteração é dominada por operações $O(L)$. Os experimentos realizados com esse algoritmo mostraram que, na prática, o número de iterações necessárias para a convergência do algoritmo é 
uma função $O\left(n^{2}\right)$. Então, podemos afirmar que, empiricamente, o tempo médio de execução do Algoritmo 1 é uma função $O\left(n^{2} L\right)$.

Até aqui foi mostrado que o Algoritmo 1 converge obrigatoriamente em algum ponto. Agora será investigado se o método descrito acima resulta sempre em uma solução ótima, ou seja, se a transformação encontrada resulta na menor redundância (multi-informação) possível entre as variáveis latentes encontradas. Uma possível limitação do método é o fato de trabalhar somente com um par de variáveis a cada iteração. A seguir, avaliaremos se é possível que exista uma transformação ótima envolve três variáveis simultaneamente, mas que não possa ser atingida por meio de passos intermediários envolvendo quaisquer pares.

Defina-se uma tripla de variáveis binárias, $X_{s}, X_{t}, X_{u} \in\{0,1\}$. O espaço amostral para uma tripla assim definida contém $2^{3}=8$ elementos e a distribuição conjunta $P\left(X_{s}, X_{t}, X_{u}\right)$ é totalmente determinada por 7 parâmetros. A princípio pode parecer necessário haver um parâmetro para cada elemento $\left(x_{s}, x_{t}, x_{u}\right) \in$ $\{0,1\}^{3}$, mas um dos parâmetros é determinado pela restrição:

$$
\sum_{\left(x_{s}, x_{t}, x_{u}\right) \in\{0,1\}^{3}} P\left(X_{s}, X_{t}, X_{u}\right)=1 .
$$

De modo geral, a distribuição conjunta de $n$ variáveis binárias requer a determinação de $2^{n}-1$ parâmetros. Por trabalhar com pares de variáveis, o método descrito acima pode manipular apenas as distribuições marginais $P\left(X_{s}, X_{t}\right), P\left(X_{s}, X_{u}\right)$, $P\left(X_{t}, X_{u}\right)$. O conjunto dessas três distribuições marginais fornece no máximo 6 parâmetros independentes. Por exemplo, as três distribuições são completamente determinadas por: $P\left(X_{s}=1\right), P\left(X_{t}=1\right), P\left(X_{u}=1\right), P\left(X_{s}=1, X_{t}=1\right)$, $P\left(X_{s}=1, X_{u}=1\right), P\left(X_{t}=1, X_{u}=1\right)$. As distribuições marginais não permitem inferir um parâmetro que relacione as três variáveis simultaneamente (por exemplo, $\left.P\left(X_{s}=1, X_{t}=1, X_{u}=1\right)\right)$

Seja $P\left(X_{s}, X_{t}, X_{u}\right)$ uma distribuição tal que a redundância entre as três variáveis seja mínima, isto é, não exista matriz de paridade $\mathbf{W}$ tal que a multiinformação entre as variáveis $\left(Z_{s}, Z_{t}, Z_{u}\right)$ obtidas pela transformação $\left[Z_{s}, Z_{t}, Z_{u}\right]^{T}=$ $\mathbf{W}\left[X_{s}, X_{t}, X_{u}\right]^{T}$ seja menor que a multi-informação entre $\left(X_{s}, X_{t}, X_{u}\right)$. Vamos denominar uma distribuição com essa característica MI-mínima. Em princípio, seria possível construir uma distribuição $P^{\prime}\left(X_{s}, X_{t}, X_{u}\right)$ com as mesmas distri- 
buições marginais que $P\left(X_{s}, X_{t}, X_{u}\right)$, mantendo-se os 6 parâmetros que controlam as distribuições marginais e manipulando-se apenas o sétimo parâmetro (por exemplo, $P\left(X_{s}=1, X_{t}=1, X_{u}=1\right)$ ). Nesse novo cenário, pode existir uma matriz $\mathbf{W}$ tal que a variável aleatória $X_{\gamma}=X_{s} \oplus X_{t} \oplus X_{u}$ tenha entropia menor que alguma das variáveis originais, ou seja,

$$
H\left(X_{\gamma}\right)<\max \left(H\left(X_{s}\right), H\left(X_{t}\right), H\left(X_{u}\right)\right)
$$

O método de ICA descrito anteriormente não seria capaz de achar tal matriz $\mathbf{W}$, pois utiliza apenas as distribuições marginais. Denominaremos uma distribuição com a característica de $P^{\prime}\left(X_{s}, X_{t}, X_{u}\right)$ como MI-2-mínima pois não existe nenhuma transformação envolvendo apenas pares de variáveis que reduza a redundância (multi-informação) das variáveis. O conjunto das distribuições MI-2mínimas engloba o conjunto das distribuições MI-mínimas.

O raciocínio acima mostra que nem sempre o método proposto consegue encontrar a melhor transformação. Um exemplo é o caso em que $X_{s}$ e $X_{t}$ são variáveis independentes, com:

$$
P\left(X_{s}=1\right)=P\left(X_{t}=1\right)=0.5
$$

e

$$
X_{u}=X_{t} \oplus X_{s}
$$

Nesse caso, temos:

$$
H\left(X_{s}\right)=H\left(X_{t}\right)=H\left(X_{u}\right)=1 \text { bit. }
$$

No entanto, $H\left(X_{s} \oplus X_{t} \oplus X_{u}\right)=0$, pois $X_{s} \oplus X_{t} \oplus X_{u}$ é igual a zero para quaisquer valores de $X_{s}, X_{t}, X_{u}$ (ver Tabela 4.5).

A fim de investigar os casos em que uma distribuição é MI-2-mínima mas não MI-mínima (ou seja, em que o método proposto não pode achar a solução ótima) vamos antes adotar uma notação abreviada, em que $\tau_{s t u} \triangleq P\left(X_{s}=1, X_{t}=\right.$ $\left.1, X_{u}=1\right), \tau_{s t} \triangleq P\left(X_{s}=1, X_{t}=1\right), \tau_{s} \triangleq P\left(X_{s}=1\right)$ e assim por diante. Assim, a distribuição $P\left(X_{s}, X_{t}, X_{u}\right)$ pode ser definida pelos parâmetros $\tau_{s}, \tau_{t}, \tau_{u}$, 


\begin{tabular}{|c|c|c|c|c|}
\hline$X_{s}$ & $X_{t}$ & $X_{u}$ & $X_{s} \oplus X_{t} \oplus X_{u}$ & Prob \\
\hline 0 & 0 & 0 & 0 & 0.25 \\
\hline 0 & 1 & 1 & 0 & 0.25 \\
\hline 1 & 0 & 1 & 0 & 0.25 \\
\hline 1 & 1 & 0 & 0 & 0.25 \\
\hline
\end{tabular}

Tabela 4.5: Valores possíveis para a tripla $\left(X_{s}, X_{t}, X_{u}\right)$ e respectivas probabilidades. $X_{s}$ e $X_{t}$ são independentes, com $P\left(X_{s}=1\right)=P\left(X_{t}=1\right)=0.5, X_{u}$ é determinada pela função $X_{u}=X_{s} \oplus X_{t}$. Decorre que $X_{s} \oplus X_{t} \oplus X_{u}=0$.

$\tau_{s t}, \tau_{s u}, \tau_{t u}, \tau_{s t u}$.

Sem perda de generalidade, podemos adotar

$$
\tau_{u}, \tau_{t}, \tau_{s} \leq 0.5
$$

Tal situação pode ser sempre obtida pela inversão Booleana da variável (i.e. caso $P(Z=1)>0.5$ substituí-se a variável $Z$ por seu inverso). Como visto anteriormente, a simples inversão não altera a multi-informação entre as variáveis. Do mesmo modo, podemos adotar uma ordem arbitrária entre os parâmetros:

$$
\tau_{u} \leq \tau_{t} \leq \tau_{s}
$$

Tais restrições permitem a simplificação da análise a seguir sem modificar qualitativamente seus resultados. Com a parametrização adotada, a distribuição marginal $P\left(X_{s}, X_{t}\right)$ pode ser expressa pela seguinte tabela de contingência 4.6 .

\begin{tabular}{|c|c|c|}
\hline & $X_{t}=0$ & $X_{t}=1$ \\
\hline$X_{s}=0$ & $1-\tau_{s}-\tau_{t}+\tau_{s t}$ & $\tau_{t}-\tau_{s t}$ \\
\hline$X_{s}=1$ & $\tau_{s}-\tau_{s t}$ & $\tau_{s t}$ \\
\hline
\end{tabular}

Tabela 4.6: Tabela de contingência para $P\left(X_{s}, X_{t}\right)$

Para que a distribuição seja MI-2-mínima é necessário que a multi-informação entre $X_{s}$ e $X_{t}$ seja mínima, o que significa que uma das diagonais da tabela de contingência deve ser formada pelo maior e o menor valores da tabela. A desigualdade 4.33 impõe que a distribuição seja mais concentrada em torno de $X_{s}=0$ 
e $X_{t}=0$. Por isso, temos que o maior valor da tabela é $1-\tau_{s}-\tau_{t}-\tau_{s t}$ e o menor é $\tau_{s t}$, implicando:

$$
\begin{gathered}
1-\tau_{s}-\tau_{t}+\tau_{s t} \geq \tau_{s}-\tau_{s t} \\
1-\tau_{s}-\tau_{t}+\tau_{s t} \geq \tau_{t}-\tau_{s t} \\
\tau_{s}-\tau_{s t} \geq \tau_{s t} \\
\tau_{t}-\tau_{s t} \geq \tau_{s t} .
\end{gathered}
$$

As mesmas considerações podem ser feitas para as distribuições marginais $P\left(X_{s}, X_{u}\right)$ e $P\left(X_{t}, X_{u}\right)$, obtendo-se condições análogas:

$$
\begin{gathered}
1-\tau_{s}-\tau_{u}+\tau_{s u} \geq \tau_{s}-\tau_{s u} \\
1-\tau_{s}-\tau_{u}+\tau_{s u} \geq \tau_{u}-\tau_{s u} \\
\tau_{s}-\tau_{s u} \geq \tau_{s u} \\
\tau_{u}-\tau_{s u} \geq \tau_{s u} \\
1-\tau_{t}-\tau_{u}+\tau_{t u} \geq \tau_{t}-\tau_{t u} \\
1-\tau_{t}-\tau_{u}+\tau_{t u} \geq \tau_{u}-\tau_{t u} \\
\tau_{t}-\tau_{t u} \geq \tau_{t u} \\
\tau_{u}-\tau_{t u} \geq \tau_{t u} .
\end{gathered}
$$

Usando a restrição (4.34) e as inequações 4.35) e 4.36 temos:

$$
\tau_{s t} \geq \tau_{s}+\tau_{t} / 2-1 / 2
$$

Do mesmo modo, restrição (4.34), inequações 4.39) e (4.40) implicam:

$$
\tau_{s u} \geq \tau_{s}+\tau_{u} / 2-1 / 2
$$

E restrição (4.34), inequações 4.43) e (4.44) implicam:

$$
\tau_{t u} \geq \tau_{t}+\tau_{u} / 2-1 / 2
$$


Novamente usando a restrição 4.34, junto com as inequações 4.37) e 4.38), temos:

$$
\tau_{s t} \leq \tau_{t} / 2
$$

De maneira análoga, pode-se mostrar que:

$$
\tau_{s u} \leq \tau_{u} / 2
$$

$\mathrm{e}$

$$
\tau_{\text {tu }} \leq \tau_{u} / 2
$$

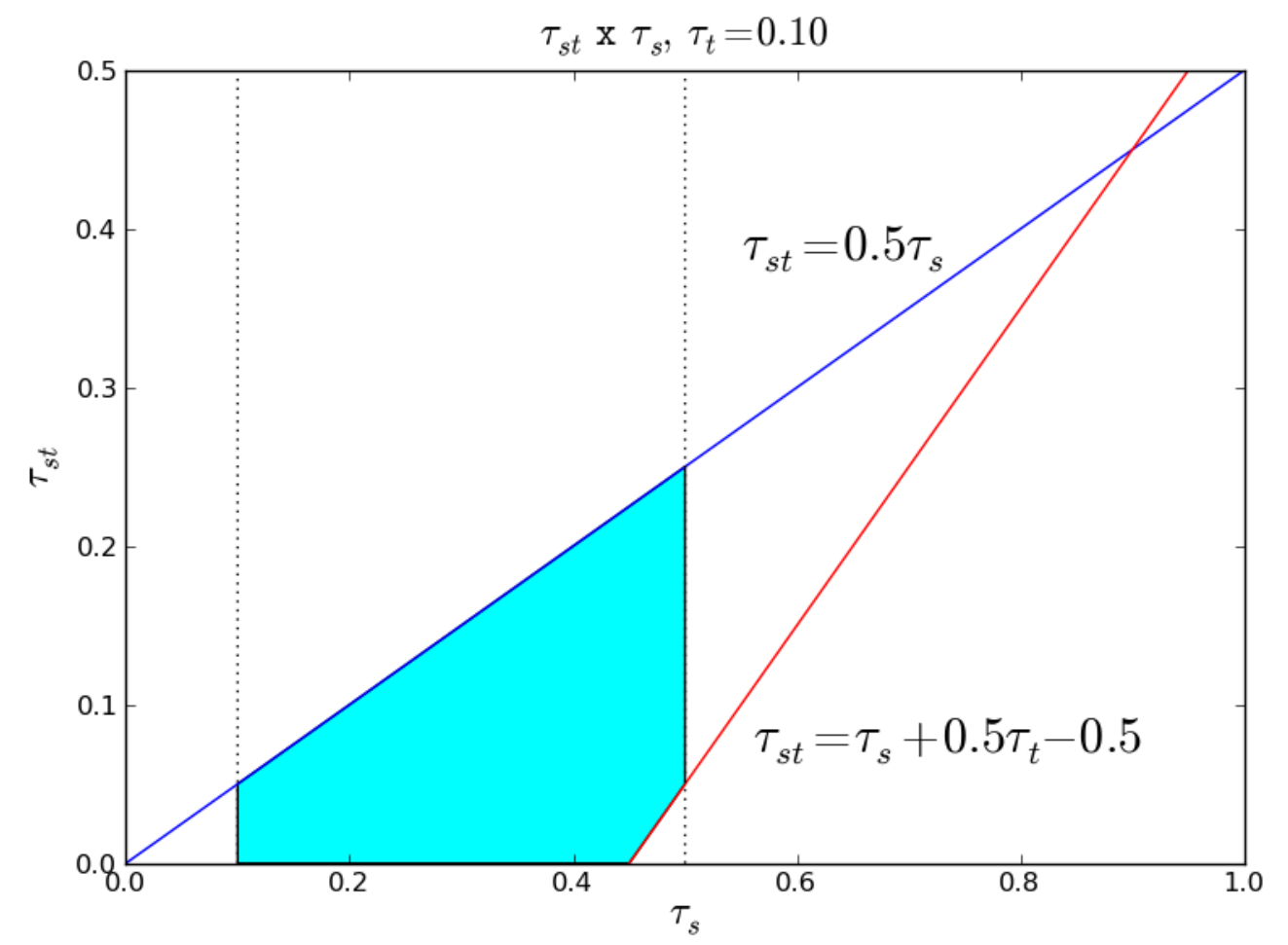

Figura 4.1: Região permitida para $\tau_{s t}$ quando $\tau_{t}=0.1$. As duas linhas pontilhadas verticais demarcam a região $\tau_{t} \leq \tau_{s}<1 / 2$. A área colorida mostra os valores que $\tau_{s t}$ pode assumir.

O conjunto de inequações 4.47), 4.48), 4.49), 4.50, 4.51) e 4.52) mostram como a condição de distribuição MI-2-mínima afeta o espaço de parâmetros das distribuições marginais. As Figuras 4.1 e 4.2 ilustram o efeito dessas restrições 


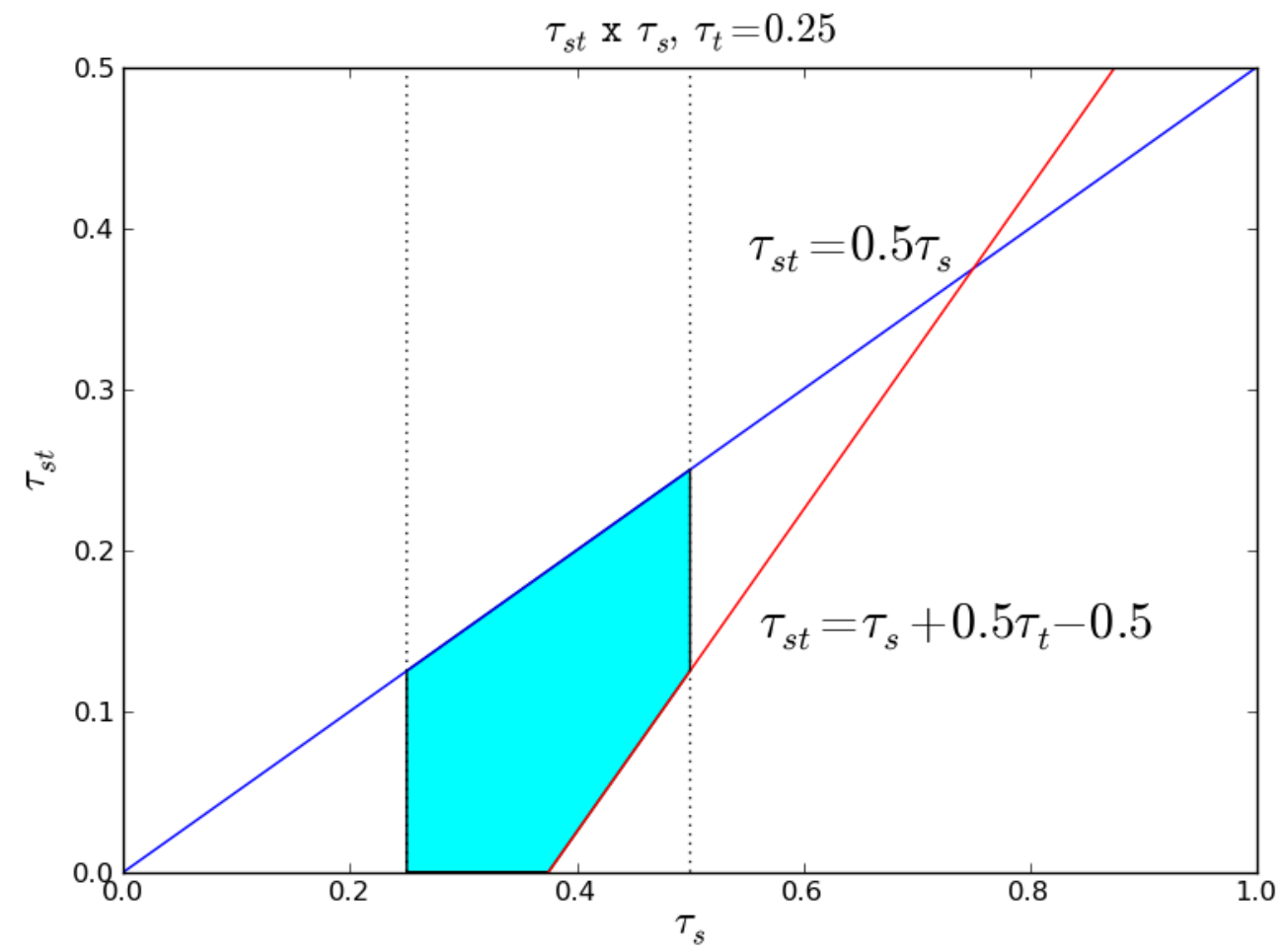

Figura 4.2: Região permitida para $\tau_{s t}$ quando $\tau_{t}=0.25$. As duas linhas pontilhadas verticais demarcam a região $\tau_{t} \leq \tau_{s}<1 / 2$. A área colorida mostra os valores que $\tau_{s t}$ pode assumir.

sobre $\tau_{s t}$ em função de $\tau_{s}$, considerando o valor de $\tau_{t}$ fixado primeiro em $\tau_{t}=0.1$ e depois $\tau_{t}=0.25$. Na comparação das duas figuras nota-se que o espaço em que $\tau_{s t}$ pode variar, para uma distribuição MI-2-mínima, diminui conforme $\tau_{t}$ aumenta em direção ao seu limite máximo, $1 / 2$. Comportamento análogo se verifica para $\tau_{s u}$ e $\tau_{t u}$.

Em resumo, a análise feita até agora aponta que as distribuições MI-2-mínimas ocupam uma porção pequena do espaço de parâmetros possíveis para distribuições de probabilidade com três variáveis binárias. Dentro do espaço ocupado pelas distribuições MI-2-mínimas estão localizadas as distribuições que são também MI-mínimas. Estas últimas não oferecem problemas para o algoritmo de ICA binária apresentado.

Para avaliar a importância relativa das distribuições MI-2-mínimas e MI-mínimas 
dentro do espaço de parâmetros foi realizado um experimento de Monte Carlo. Nesse experimento, um determinado número de distribuições $P\left(X_{s}, X_{t}, X_{u}\right)$ foi gerado aleatoriamente, amostrando-se de maneira uniforme o espaço de parâmetros das distribuições binárias contendo três variáveis. Para cada distribuição gerada foi verificado em qual categoria a distribuição se encaixava (MI-mínima, apenas MI-2-mínima, não mínima), mantendo-se uma contagem para cada categoria. Um total de 100.000 distribuições foram geradas. A parametrização escolhida para gerar as distribuições foi definida em termos de probabilidades condicionais, sendo adotados os sete parâmetros a seguir:

$$
\begin{gathered}
P\left(X_{s}=1\right) \\
P\left(X_{t}=1 \mid X_{s}=1\right) \\
P\left(X_{t}=1 \mid X_{s}=0\right) \\
P\left(X_{u}=1 \mid X_{t}=1, X_{s}=1\right) \\
P\left(X_{u}=1 \mid X_{t}=1, X_{s}=0\right) \\
P\left(X_{u}=1 \mid X_{t}=0, X_{s}=1\right) \\
P\left(X_{u}=1 \mid X_{t}=0, X_{s}=0\right) .
\end{gathered}
$$

A parametrização em termos de distribuições condicionais foi adotada para facilitar a amostragem dos parâmetros, por permitir que os parâmetros variem independentemente, dentro de um intervalo fixo [0,1], enquanto os parâmetros $\tau$ exibem vínculos entre si (como mostram, por exemplo, as Figuras $4.1 \mathrm{e} 4.2$ ).

A Tabela 4.1 mostra a divisão do espaço de parâmetros de $P\left(X_{s}, X_{t}, X_{u}\right)$ por tipo de distribuição. A maior parte do espaço $(92,7 \%)$ é ocupada por distribuições não-mínimas, para as quais o Algoritmo 1 é capaz de encontrar uma transformação que reduza a multi-informação. Entre os casos em que o algoritmo não é capaz de encontrar uma transformação adequada, as distribuições MI-mínimas ainda são cerca de 4 vezes mais frequentes que as distribuições apenas MI-2-mínimas (5,9\% contra 1,4\%). Quando a distribuição é apenas MI-2-mínima o algoritmo oferece uma solução não ótima. Nessa situação, a perda em termos de multi-informação 


\begin{tabular}{l|l} 
MI-mínima & $5,9 \%$ \\
\hline MI-2 mínima apenas & $1,4 \%$ \\
\hline não mínima & $92,7 \%$
\end{tabular}

Tabela 4.7: Divisão do espaço de parâmetros de $P\left(X_{s}, X_{t}, X_{u}\right)$ por tipo de distribuição. A maior parte do espaço é ocupada por distribuições não-mínimas. As distribuições MImínimas são cerca de 4 vezes mais frequentes que as distribuições apenas MI-2-mínimas.

em relação à solução ótima é dada pela seguinte diferença

$$
\text { perda } \mathrm{MI}=\max \left(H\left(X_{s}\right), H\left(X_{t}\right), H\left(X_{u}\right)\right)-H\left(X_{s} \oplus X_{t} \oplus X_{u}\right)
$$

pois a variável de maior entropia deveria ser substituída por $X_{s} \oplus X_{t} \oplus X_{u}$ para obtermos a solução ótima. Essa perda pode ser avaliada na Figura 4.3, que mostra $H\left(X_{s} \oplus X_{t} \oplus X_{u}\right)$ em função de $\max \left(H\left(X_{s}\right), H\left(X_{t}\right), H\left(X_{u}\right)\right)$ para as distribuições simuladas em que o algoritmo não encontra solução ótima. A linha contínua diagonal corresponde função à identidade, isto é:

$$
H\left(X_{s} \oplus X_{t} \oplus X_{u}\right)=\max \left(H\left(X_{s}\right), H\left(X_{t}\right), H\left(X_{u}\right)\right)
$$

A maior parte dos pontos se concentra junto a essa linha, indicando que a diferença entre a solução ótima e a solução para qual o algoritmo converge tende a ser pequena. A Figura 4.4 traz o histograma e a distribuição acumulada da perda de multi-informação para os pontos mostrados na Figura 4.3 , confirmando que a maior concentração ocorre perto de zero.

Antes de prosseguir com a análise, é preciso dizer que o experimento de Monte Carlo realizado e os números e gráficos resultantes refletem características do espaço das distribuições de triplas de variáveis binárias, não necessariamente das distribuições observadas na prática, com dados reais. De fato, há razão para supor que dados provenientes de medições reais apresentam distribuições bastante particulares [OF96, OF97, BS97, HH01, HH00, HH02, Pec02, BGDA04, ZW04], indicando que uma parte expressiva do espaço das distribuições é quase impossível de ser observada na prática. No entanto, a análise mostrou a possibilidade de existirem transformações ótimas envolvendo triplas de variáveis que não seriam 
selecionadas pelo Algoritmo 1. O mesmo raciocínio aplicado para as triplas pode ser extrapolado para conjuntos maiores de variáveis, acarretando a mesma conclusão: o conjunto de distribuições marginais de pares de variáveis não contém parâmetros suficientes para especificar as distribuições de ordem superior, sendo sempre possível fabricar uma distribuição $P\left(X_{1}, X_{2}, \ldots, X_{n}\right), n>2$, para a qual o algoritmo não é capaz de encontrar a transformação ótima. Na próxima seção serão apresentados experimentos com dados simulados, visando avaliar a perda observada na prática.

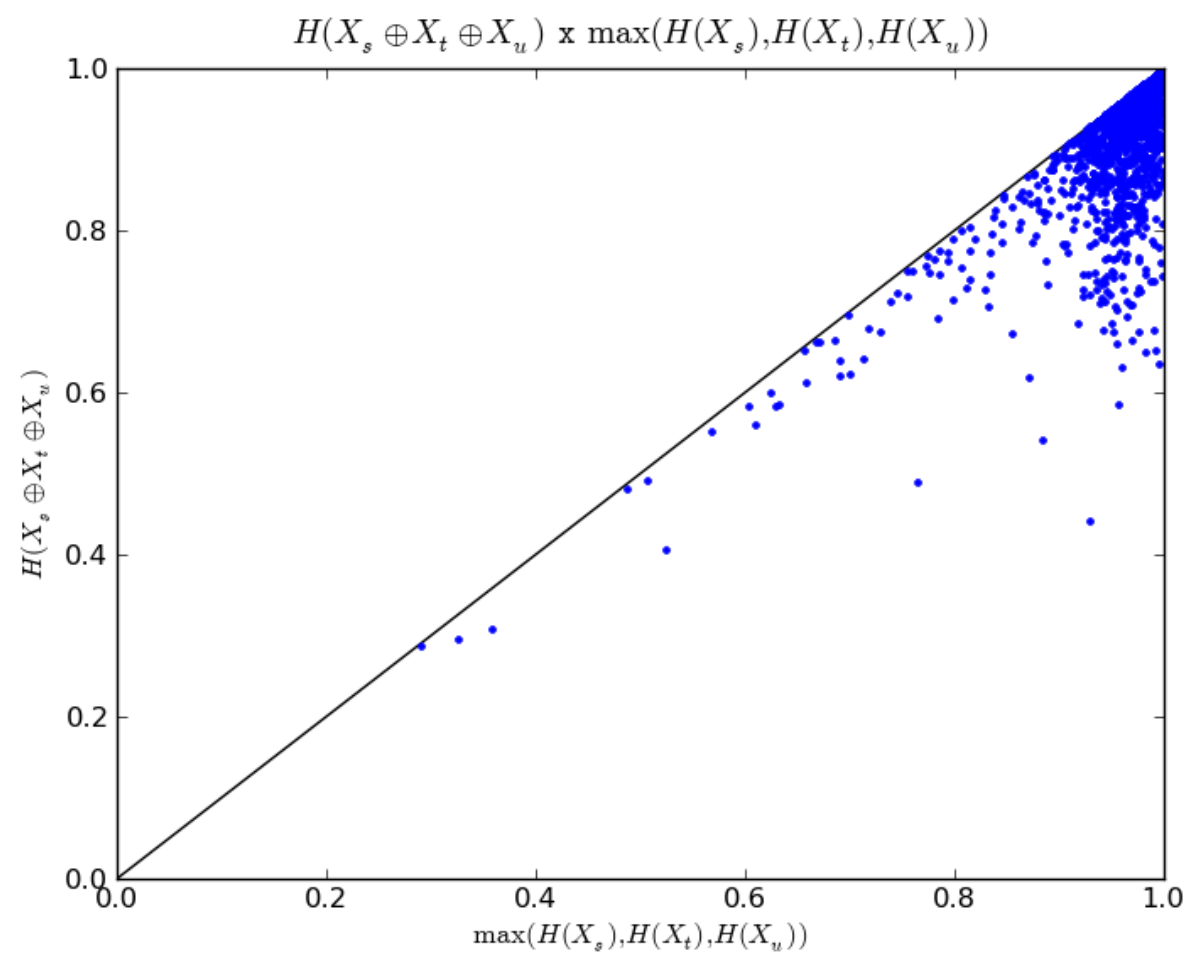

Figura 4.3: Gráfico de dispersão de $H\left(X_{s} \oplus X_{t} \oplus X_{u}\right)$ em função de $\max \left(H\left(X_{s}\right), H\left(X_{t}\right), H\left(X_{u}\right)\right)$ para as distribuições que são MI-2-mínimas mas não MI-mínimas. A linha contínua diagonal corresponde a $H\left(X_{s} \oplus X_{t} \oplus X_{u}\right)=$ $\max \left(H\left(X_{s}\right), H\left(X_{t}\right), H\left(X_{u}\right)\right)$. Para cada ponto, a perda incorrida corresponde à distância vertical entre o ponto e a linha contínua. 

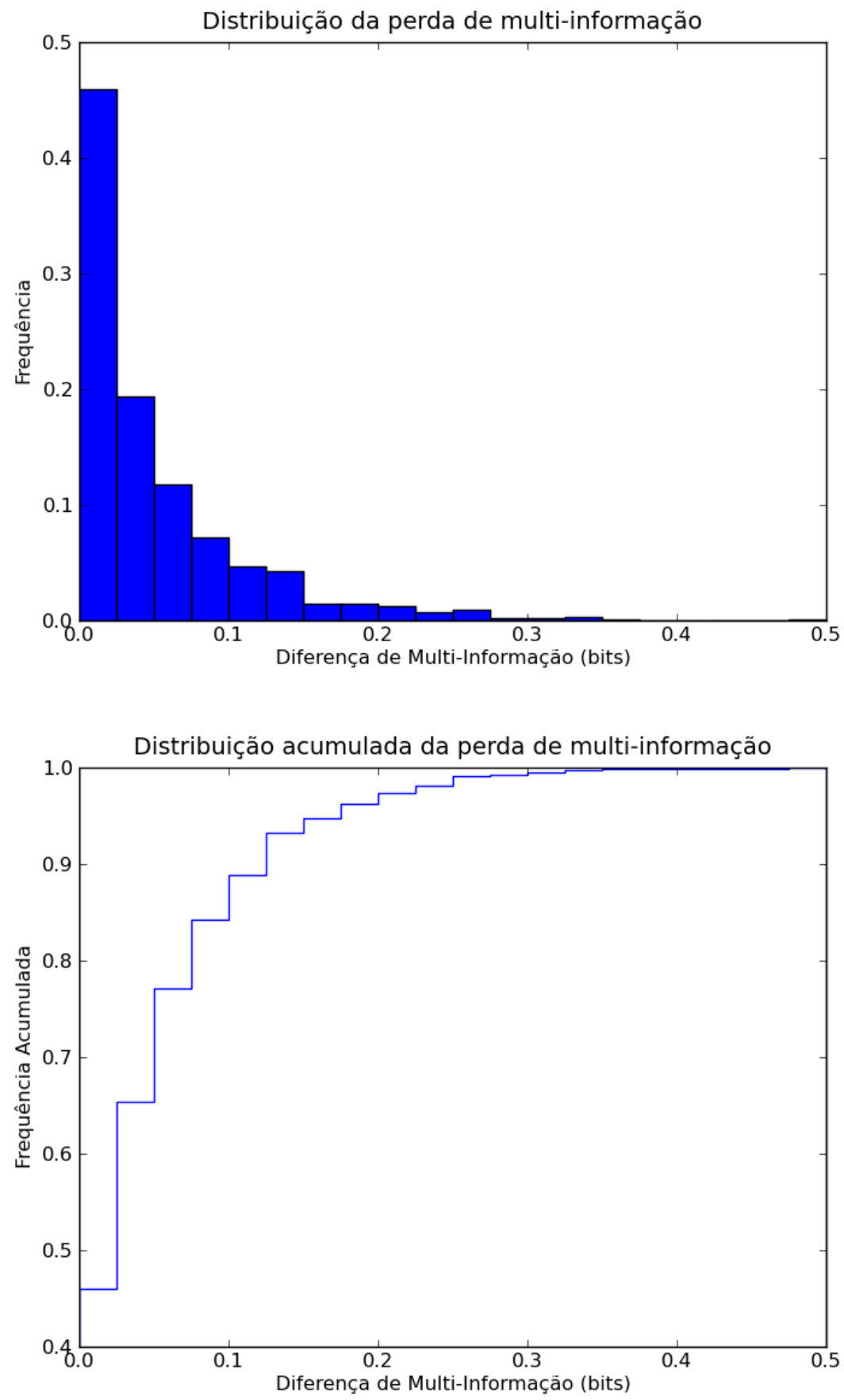

Figura 4.4: Histogramas da perda de multi-informação para as situações em que Algoritmo 1 não consegue encontrar uma solução ótima. 


\subsection{Experimentos com o algoritmo de ICA binária}

A fim de testar o Algoritmo 1 quanto à sua capacidade de minimizar a multiinformação de um conjunto de variáveis foram realizados experimentos com dados simulados, medindo-se de maneira indireta a multi-informação dos dados simulados e dos dados obtidos pela execução do Algoritmo 1. O modelo para a geração dos dados simulados será descrito a seguir: inicialmente são geradas realizações independentes de um vetor $\mathbf{Z} \in\{0,1\}^{n}$. Cada elemento $Z_{i}$ de $\mathbf{Z}$ é gerado independentemente com uma probabilidade fixa $P\left(Z_{i}=1\right)=p$, logo:

$$
\forall i \in\{1,2, \ldots, n\}: H\left(Z_{i}\right)=-p \log _{2} p-(1-p) \log _{2}(1-p)=H_{p}
$$

Como os elementos $Z_{i}$ são independentes, segue que:

$$
H(\mathbf{Z})=H\left(Z_{1}, Z_{2}, \ldots, Z_{n}\right)=\sum_{i=1}^{n} H\left(Z_{i}\right)
$$

Portanto, pela definição de multi-informação (Equação 4.2,$M I(\mathbf{Z})=0$. Cada elemento de $\mathbf{Z}$ corresponde a uma fonte binária independente. As realizações de $\mathbf{Z}$ serão denotadas por $\mathbf{z}^{(1)}, \mathbf{z}^{(2)}, \ldots, \mathbf{z}^{(L)}$.

Também é gerada aleatoriamente uma matriz $n \mathbf{x} n$ binária A que corresponde à matriz de mistura. Essa matriz é gerada pelo seguinte procedimento iterativo: inicialmente $\mathbf{A}=\mathbf{I}_{n}$. Seleciona-se então um número $m$ de passos, $m>>n$. A cada passo, um par de índices distintos $(i, j)$ é selecionado aleatoriamente e a matriz A é atualizada, substituindo-se sua $i$-ésima coluna pela soma (módulo-2) dessa coluna com a $j$-ésima. Esse procedimento garante que não haja perda de entropia conjunta quando a matriz for usada para transformar um vetor binário (essa é a condição da Equação 4.9). Desse modo poderemos comparar a multiinformação antes e depois de uma determinada transformação.

A partir de $\mathbf{A}$ e de um conjunto de realizações de $\mathbf{Z}$, aplica-se a transformação $\mathbf{X}=\mathbf{A Z}$, obtendo-se o conjunto de realizações de $\mathbf{X}$, o vetor observado que servirá de entrada para o algoritmo de ICA binária. As realizações de $\mathrm{X}$ serão 
denotadas por $\mathbf{x}^{(1)}, \mathbf{x}^{(2)}, \ldots, \mathbf{x}^{(L)}$, valendo a relação

$$
\mathbf{x}^{(l)}=\mathbf{A} \mathbf{z}^{(l)}
$$

A transformação $\mathbf{X}=\mathbf{A Z}$ introduz uma dependência entre os elementos de $\mathbf{X}$, de modo que $M I(\mathbf{X})>0$.

Em seguida, Algoritmo 1 é aplicado, tendo as realizações de $\mathrm{X}$ como entrada. A saída da execução do algoritmo é constituída pela matriz de separação estimada $\hat{\mathbf{W}}$ e por um conjunto de vetores $\hat{\mathbf{z}}^{(1)}, \hat{\mathbf{z}}^{(2)}, \ldots, \hat{\mathbf{z}}^{(L)}$ que são realizações de $\hat{\mathbf{Z}}$, valendo a relação:

$$
\hat{\mathbf{z}}^{(l)}=\hat{\mathbf{W}} \mathbf{x}^{(l)} .
$$

Por construção, $\hat{\mathbf{W}}$ também preserva a entropia conjunta quando usada para transformar um vetor. Isso nos permite afirmar que:

$$
H(\hat{\mathbf{Z}})=H(\mathbf{X})=H(\mathbf{Z})
$$

Usando a definição de multi-informação (Equação 4.2), pode-se concluir que:

$$
M I(\hat{\mathbf{Z}})-M I(\mathbf{Z})=\sum_{i=0}^{n} H\left(\hat{Z}_{i}\right)-\sum_{i=0}^{n} H\left(Z_{i}\right) .
$$

A Equação 4.56 mede a multi-informação residual que o algoritmo não é capaz de eliminar. Idealmente teríamos $M I(\hat{\mathbf{Z}})-M I(\mathbf{Z})=0$.

Do mesmo modo, a expressão:

$$
M I(\mathbf{X})-M I(\hat{\mathbf{Z}})=\sum_{i=0}^{n} H\left(X_{i}\right)-\sum_{i=0}^{n} H\left(\hat{Z}_{i}\right)
$$

mede a redução de multi-informação devida à execução do algoritmo.

Nos experimentos realizados, adotou-se $n=20$. Para testar a robustez do algoritmo na presença de ruído, o Algoritmo 1 não foi aplicado diretamente sobre $\mathrm{X}$ mas sim sobre

$$
\mathbf{X}^{\prime}=\mathbf{X}+\mathbf{R},
$$

onde $\mathbf{R} \in\{0,1\}^{n}$ é um vetor aleatório de ruído e a soma é módulo-2. Cada 
componente de $\mathbf{R}$ é gerado de maneira independente, com probabilidade $P\left(R_{i}=\right.$ $1)=p_{R}$. Nos experimentos, foram utilizados os valores 0.00, 0.01 e 0.02 para $p_{R}$. Também se variou, nos experimentos, o valor da probabilidade $p$ que caracteriza as fontes independentes. Foram utilizados os seguintes valores para $p$ : 0.05, 0.07, $0.09,0.12,0.15,0.22,0.3$ e 0.5 . Para cada combinação de $p$ e $p_{R}$ foi utilizada uma matriz $\mathbf{A}$ diferente. Então, para cada tripla $\left(p, p_{R}, \mathbf{A}\right)$, repetiu-se 10 vezes o procedimento de gerar a amostra de $\mathbf{X}$ e $\mathbf{R}$ e aplicar-se o Algoritmo 1 . O tamanho do conjunto de treinamento utilizado foi $L=100$. Em cada uma das repetições, calculou-se o valor $\sum_{i=1}^{n} H\left(Z_{i}\right)$ pela fórmula 4.53) e mediram-se empiricamente os valores $\sum_{i=1}^{n} H\left(\hat{Z}_{i}\right)$ e $\sum_{i=1}^{n} H\left(X_{i}\right)$.

Os resultados dos experimentos são apresentados no formato de diagrama de caixa (boxplot) pela Figura 4.5 para $p_{R}=0$, Figura 4.6 para $p_{R}=0.01$ e Figura 4.7 para $p_{R}=0.02$. Cada figura contém 2 gráficos. O gráfico de cima mostra a variação de $\sum_{i=1}^{n} H\left(\hat{Z}_{i}\right)$ em função de $\sum_{i=1}^{n} H\left(Z_{i}\right)$. Nesse gráfico, a linha diagonal contínua representa a função identidade, permitindo-nos avaliar visualmente a diferença

$$
\sum_{i=1}^{n} H\left(\hat{Z}_{i}\right)-\sum_{i=1}^{n} H\left(Z_{i}\right)
$$

Idealmente, a diferença acima seria nula é os valores obtidos para $\sum_{i=1}^{n} H\left(\hat{Z}_{i}\right)$ se concentrariam próximos à linha da função identidade. Constata-se que o desempenho do algoritmo degrada-se conforme o nível de ruído aumenta, comparando-se os gráficos das Figuras 4.5, 4.6 e 4.7, que apresentam nível de ruído crescente. Tomando um valor fixo de $\sum_{i=1}^{n} H\left(Z_{i}\right)$ (por exemplo, 7.3), nota-se que o valor de $\sum_{i=1}^{n} H\left(\hat{Z}_{i}\right)$ aumenta em função do aumento do ruído.

$\mathrm{O}$ algoritmo também funciona melhor quando os dados são esparsos, i.e. altamente concentrados em um único valor, e a somatória $\sum_{i=1}^{n} H\left(Z_{i}\right)$ é menor. De fato, o valor da somatória $\sum_{i=1}^{n} H\left(\hat{Z}_{i}\right)$ somente se aproximam do valor ideal para os primeiros três pontos da Figura 4.5, para os quais $p=0.05, p=0.07 \mathrm{e}$ $p=0.09$, respectivamente, sendo o ruído nulo.

Adicionalmente, o gráfico de baixo em cada uma das figuras referidas mostra $\sum_{i=1}^{n} H\left(X_{i}\right)-\sum_{i=1}^{n} H\left(\hat{Z}_{i}\right)$, quantificando o decréscimo de multi-informação 
devido à execução do algoritmo. Observa-se que mesmo quando o algoritmo não é capaz de se aproximar do resultado ideal existe algum decréscimo da multiinformação, mas a capacidade de reduzir a multi-informação também se degrada consideravelmente na presença de ruído.

Antes de analisar o impacto dos resultados acima sobre a aplicabilidade do Algoritmo 1 é preciso considerar os possíveis cenários de uso do mesmo. As aplicações de ICA para dados contínuos podem ser divididas em dois grupos. No primeiro grupo, o modelo adotado (fontes independentes combinadas linearmente) é uma hipótese realista sobre o sinal analisado. Nesse grupo se enquadram alguns casos de análise de sinais de áudio [ [BS96] e o problema da separação cega de fontes [BS95]. No segundo grupo, o modelo linear não é tratado como uma reprodução fiel do processo de geração do sinal, mas como um instrumento útil para se entender certas características dos dados. Isso acontece, por exemplo, em algumas aplicações de análise de imagens [BS97][HHI02][BMS02]. Do mesmo modo que ocorre com esse segundo grupo de aplicações, o método proposto neste capítulo deve ser enxergado como um meio de extrair informações sobre os dados analisados e/ou mudar a representação dos dados para uma forma mais conveniente. A execução do Algoritmo 1 produz a matriz de separação $\mathbf{W}$ e o vetor de variáveis latentes Z . Não há, a princípio, motivo para interpretar os componentes de $\mathbf{Z}$ como "fontes" que se combinam para formar o sinal observado $\mathbf{X}$, pois a independência entre os componentes $Z_{i}$ não pode ser assegurada e também porque é difícil imaginar uma situação não-artificial em que fontes binárias se combinassem por meio de uma matriz de paridade desconhecida.

A transformação $\mathbf{Z}=\mathbf{W X}$ deve ser interpretada como uma mudança de coordenadas em $\{0,1\}^{n}$, tendo como efeito uma representação mais esparsa [OF97, Pec02, $\mathrm{KC03}]$, isto é, as variáveis aleatórias resultantes $Z_{i}$ são mais concentradas em um único valor, em comparação às variáveis originais $X_{i}$. A esparsidade já foi apontada como um traço desejável para a representação de sinais, apresentando características como invariâncias de fase e deslocamento [OF96, HH00], decomposição de um objeto em partes [Hoy04] e aumento da capacidade de memórias auto-associativas [Ama89, OF04]. Em particular, o Algoritmo 1 se prestaria a aumentar a capacidade de Redes de Hopfield [Hop82, Hop07], um tipo de rede neural recursiva cujos nós correspondem a variáveis aleatórias binárias, utilizada 
como memória auto-associativa. Nesse caso, o aumento da esparsidade pode ser benéfico para a aplicação, mesmo que a execução do Algoritmo 1 não possa garantir a representação ótima com relação à multi-informação.

Outro cenário de uso do Algoritmo 1 envolve os grupos de variáveis que se formam durante sua execução. Esses grupos reúnem variáveis aleatórias que são estatisticamente dependentes entre si. A análise de tais grupos pode ser útil no contexto de análise exploratória de dados ou para a aprendizagem da estrutura de modelos gráficos [Jor04], caso se deseje modelar a distribuição dos dados. Em ambos os casos, o fato do Algoritmo 1 não fornecer a solução ótima quanto à minimização da multi-informação pode trazer prejuízos, pois os grupos deixam de refletir com fidelidade as relações de dependência entre as variáveis. 

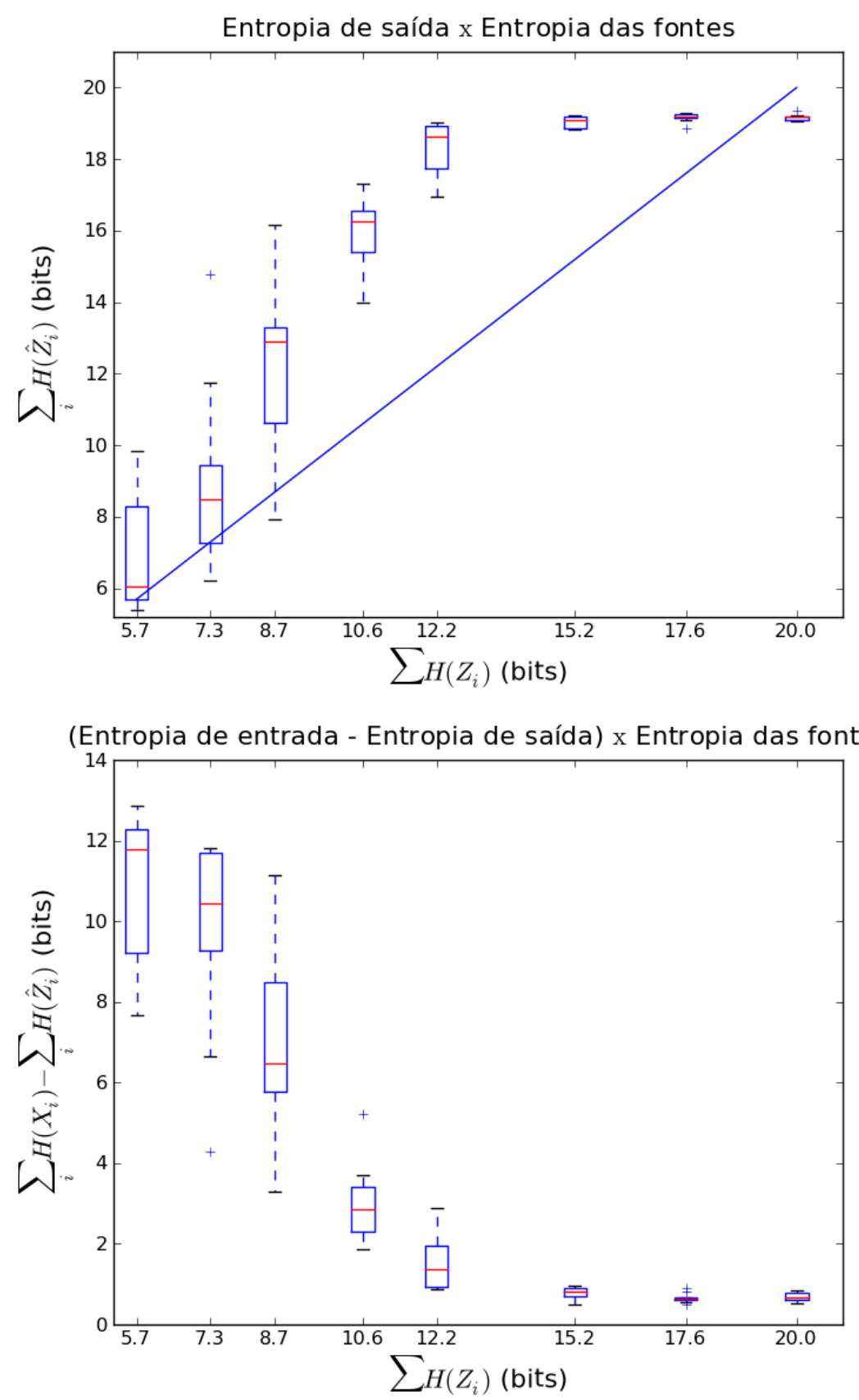

Figura 4.5: Resultados dos experimentos $\operatorname{com} p_{R}=0$. Acima: somatória de entropias das fontes comparada com somatória de entropias dos dados de saída do procedimento de ICA binária. A linha mostra a função identidade, de modo que a distância até a linha mostra a multi-informação residual, não eliminada pelo algoritmo. Em baixo, medida da multi-informação eliminada pelo algoritmo, dada pela diferença entre a soma das entropias na entrada do algoritmo e a soma das entropias na saída. 

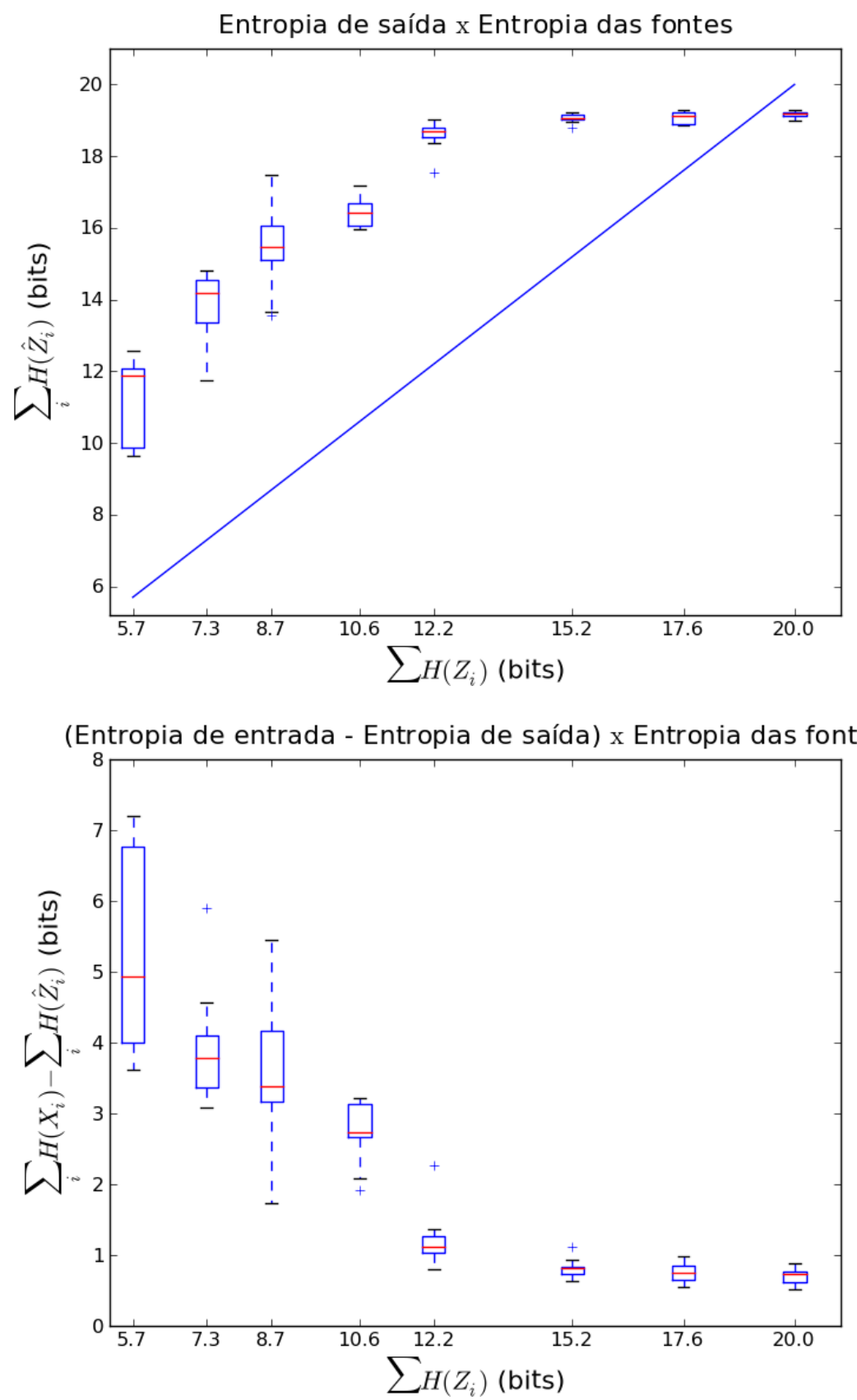

Figura 4.6: Resultados dos experimentos $\operatorname{com} p_{R}=0.01$, a disposição dos gráficos é a mesma da Figura 4.5 

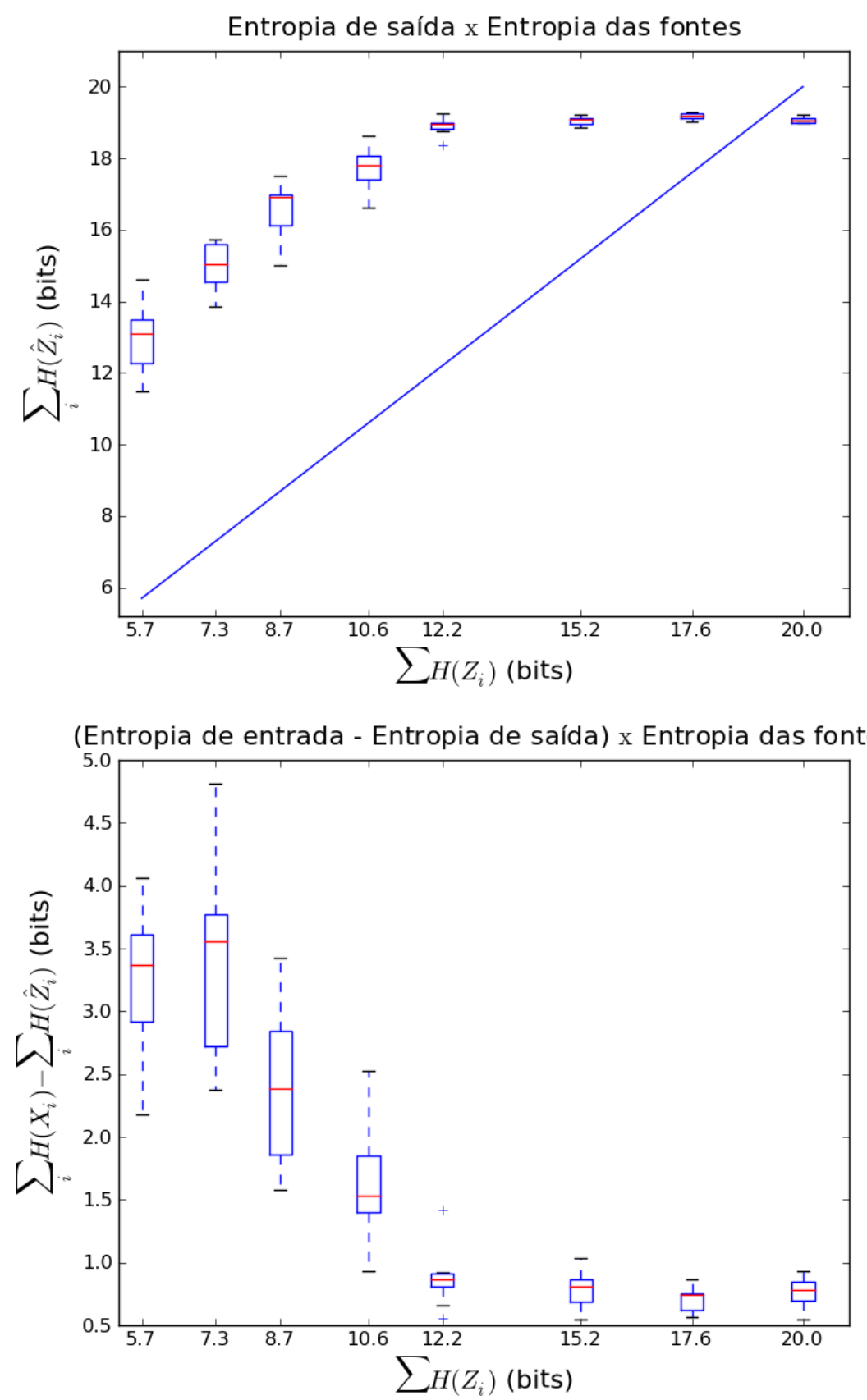

Figura 4.7: Resultados dos experimentos $\operatorname{com} p_{R}=0.02$, a disposição dos gráficos é a mesma da Figura 4.5 . 


\section{Capítulo 5}

\section{Construção de atributos por minimização da multi-informação condicional}

Neste capítulo será apresentada uma técnica nova para a construção de atributos binários para classificação, publicada pela primeira vez em [SHJ07]. Esse técnica, assim como o método de ICA binária do Capítulo 4, usa uma matriz de cálculo de paridade para transformar os atributos originais. A motivação para a técnica proposta parte da análise do classificador Naive Bayes, visando criar atributos que se adaptem melhor à hipótese de independência condicional adotada por tal classificador.

Como observado na Seção 3.5, o classificador Naive Bayes é derivado a partir de uma hipótese de independência condicional entre os atributos $X_{i}$, dado o valor do rótulo $Y$ :

$$
P\left(X_{1}, X_{2}, \ldots, X_{n} \mid Y\right)=\prod_{i=1}^{n} P\left(X_{i} \mid Y\right) .
$$

Essa é uma hipótese raramente verificada na prática e, em princípio, pode haver um erro significativo nas estimativas de probabilidade condicional calculadas adotando essa simplificação. Um meio para avaliar a distância entre o modelo de independência condicional e a distribuição verdadeira é usar a divergência KL condicional [CT91] entre a distribuição condicional verdadeira $P(X \mid Y)$ e a distribuição condicional adotada como aproximação, que denotaremos por $Q(X \mid Y)$ :

$$
D(P(X \mid Y) \| Q(X \mid Y))=\sum_{y} \sum_{x} P(x, y) \log \frac{P(x \mid y)}{Q(x \mid y)} .
$$


Adotando:

$$
Q(X \mid Y)=\prod_{i=1}^{n} P\left(X_{i} \mid Y\right)
$$

nós temos então:

$$
\begin{aligned}
D(P(X \mid Y) \| Q(X \mid Y))= & \sum_{\mathcal{X}, \mathcal{Y}} P\left(Y, X_{1}, \ldots, X_{n}\right) \log \frac{P\left(X_{1}, \ldots, X_{n} \mid Y\right)}{\prod_{i=1}^{n} P\left(X_{i} \mid Y\right)} \\
= & \sum_{\mathcal{X}, \mathcal{Y}} P\left(Y, X_{1}, \ldots, X_{n}\right) \log \frac{1}{\prod_{i=1}^{n} P\left(X_{i} \mid Y\right)} \\
& -\sum_{\mathcal{X}, \mathcal{Y}} P\left(Y, X_{1}, \ldots, X_{n}\right) \log \frac{1}{P\left(X_{1}, \ldots, X_{n} \mid Y\right)} \\
= & \sum_{\mathcal{X}, \mathcal{Y}} P\left(Y, X_{1}, \ldots, X_{n}\right) \log \frac{1}{\prod_{i=1}^{n} P\left(X_{i} \mid Y\right)} \\
& -H(\mathbf{X} \mid Y) \\
= & \sum_{\mathcal{X}, \mathcal{Y}} P\left(Y, X_{1}, \ldots, X_{n}\right) \sum_{i=1}^{n} \log \frac{1}{P\left(X_{i} \mid Y\right)} \\
& -H(\mathbf{X} \mid Y) \\
= & \sum_{i=1}^{n} \sum_{\mathcal{X}_{i}, \mathcal{Y}} P\left(Y, X_{i}\right) \log \frac{1}{P\left(X_{i} \mid Y\right)} \\
& -H(\mathbf{X} \mid Y) \\
= & \sum_{i=1}^{n} H\left(X_{i} \mid Y\right)-H(\mathbf{X} \mid Y)
\end{aligned}
$$

A expressão 5.1 mostra que a distância, medida pela divergência KL, entre a distribuição verdadeira $P(\mathbf{X} \mid Y)$ e a distribuição adotado pelo classificador Naive Bayes é dada pela multi-informação condicional entre os atributos, definida por

$$
M I(\mathbf{X} \mid Y):=\sum_{i=1}^{n} H\left(X_{i} \mid Y\right)-H(\mathbf{X} \mid Y)
$$

Dado que o método de ICA binária apresentado no Capítulo 4 opera pela minimização da multi-informação, o resultado acima sugere a possibilidade de projetarse um método semelhante para minimização da multi-informação condicional. A multi-informação condicional é uma medida da dependência residual entre os atri- 


\subsection{UM ALGORITMO PARA A REDUÇÃO DA MULTI-INFORMAÇÃO CONDICIONAL69}

butos que não pode ser explicada pela variável aleatória que faz o papel de rótulo. Em outras palavras, a multi-informação condicional expressa relações intrínsecas aos atributos. O método da transformação por matriz de paridade, apresentado no Capítulo 4, tem a característica de agrupar atributos binários. Dada uma transformação que reduzisse a multi-informação condicional, os grupos formados seriam reflexo dessas relações entre atributos, podendo revelar informações interessantes sobre os mesmos. A próxima seção trata do desenvolvimento de um algoritmo que realiza essa minimização.

\subsection{Um algoritmo para a redução da multi-informação condi- cional}

No Capítulo 4 vimos que uma transformação $\mathbf{Z}=\mathbf{W X}$, em que $\mathbf{Z}, \mathbf{X} \in$ $\{0,1\}^{n}$ e $\mathbf{W}$ é uma matriz de cálculo de paridade especialmente construída, podia resultar na redução da multi-informação, ou seja

$$
M I(\mathbf{Z})<M I(\mathbf{X})
$$

Agora será apresentado um procedimento análogo para minimizar a multi-informação condicional. Seja $\mathbf{W}$ uma matriz binária $n \mathbf{x} n$, construída de modo iterativo por meio do procedimento descrito pela Equação 4.20, aqui reproduzida:

$$
\mathbf{W}^{(k+1)}=\mathbf{B}^{(k)} \mathbf{W}^{(k)},
$$

em que $\mathbf{W}^{(0)}=\mathbf{I}_{n}$ e o efeito da multiplicação por $\mathbf{B}^{(k)}$ equivale a substituir uma linha de $\mathbf{W}^{(k)}$ pela soma dessa linha com alguma outra. Conforme demonstrado no Capítulo 4, a transformação determinada por tal matriz preserva a entropia conjunta das variáveis e podemos escrever:

$$
H(\mathbf{Z})=H(\mathbf{X})
$$

Defina-se então um vetor aumentado

$$
\mathbf{X}^{\prime}=\left(X_{1}, X_{2}, \ldots, X_{n}, Y\right)
$$


em que $Y \in\{0,1\}$. Defina-se também uma matriz binária $\mathbf{W}^{\prime},(n+1) \mathrm{x}(n+1)$, do seguinte modo:

$$
\mathbf{W}^{\prime}=\left(\begin{array}{cc}
\mathbf{W} & \mathbf{0} \\
\mathbf{0} & 1
\end{array}\right)
$$

onde 0 representa um vetor nulo de tamanho $n$. Considerando a transformação $\mathbf{Z}^{\prime}=\mathbf{W}^{\prime} \mathbf{X}^{\prime}$, é imediato constatar que

$$
\mathbf{Z}^{\prime}=\left(Z_{1}, Z_{2}, \ldots, Z_{n}, Y\right)
$$

A matriz $\mathbf{W}^{\prime}$ pode ser construída pelo mesmo tipo de procedimento iterativo que gerou a matriz $\mathbf{W}$ (o único cuidado a ser tomado é nunca substituir a última linha da matriz). Portanto:

$$
H\left(\mathbf{Z}^{\prime}\right)=H\left(\mathbf{X}^{\prime}\right)
$$

por analogia com a Equação (5.4). Podemos expandir os termos $H\left(\mathbf{X}^{\prime}\right)$ e $H\left(\mathbf{Z}^{\prime}\right)$, resultando

$$
H\left(\mathbf{X}^{\prime}\right)=H\left(X_{1}, X_{2}, \ldots, X_{n}, Y\right)
$$

$\mathrm{e}$

$$
H\left(\mathbf{Z}^{\prime}\right)=H\left(Z_{1}, Z_{2}, \ldots, Z_{n}, Y\right)
$$

Agora podemos aplicar a fórmula de decomposição da entropia conjunta (Equação 3.3, para obter

$$
H\left(X_{1}, X_{2}, \ldots, X_{n}, Y\right)=H(Y)+H\left(X_{1}, X_{2}, \ldots, X_{n} \mid Y\right)
$$

$\mathrm{e}$

$$
H\left(Z_{1}, Z_{2}, \ldots, Z_{n}, Y\right)=H(Y)+H\left(Z_{1}, Z_{2}, \ldots, Z_{n} \mid Y\right)
$$

Aplicando (5.6) e (5.7) em (5.5) resulta em:

$$
\begin{aligned}
H\left(X_{1}, X_{2}, \ldots, X_{n}, Y\right) & =H\left(Z_{1}, Z_{2}, \ldots, Z_{n}, Y\right) \\
H(Y)+H\left(X_{1}, X_{2}, \ldots, X_{n} \mid Y\right) & =H(Y)+H\left(Z_{1}, Z_{2}, \ldots, Z_{n} \mid Y\right) \\
H\left(X_{1}, X_{2}, \ldots, X_{n} \mid Y\right) & =H\left(Z_{1}, Z_{2}, \ldots, Z_{n} \mid Y\right)
\end{aligned}
$$


Escrevendo de forma mais compacta, a expressão 5.8 se torna

$$
H(\mathbf{X} \mid Y)=H(\mathbf{Z} \mid Y)
$$

Usando a definição (5.2), a diferença $M I(\mathbf{X} \mid Y)-M I(\mathbf{Z} \mid Y)$ é dada por

$$
M I(\mathbf{X} \mid Y)-M I(\mathbf{Z} \mid Y)=\sum_{i=1}^{n} H\left(X_{i} \mid Y\right)-\sum_{i=1}^{n} H\left(Z_{i} \mid Y\right)-H(\mathbf{X} \mid Y)+H(\mathbf{Z} \mid Y)
$$

Usando (5.9) em (5.10), obtemos

$$
M I(\mathbf{X} \mid Y)-M I(\mathbf{Z} \mid Y)=\sum_{i=1}^{n} H\left(X_{i} \mid Y\right)-\sum_{i=1}^{n} H\left(Z_{i} \mid Y\right)
$$

A Equação (5.11) sugere um procedimento para a minimização da multiinformação condicional dos atributos, visando aproximar a distribuição dos atributos daquela adotada pelo classificador Naive Bayes. O procedimento resultante é análogo ao Algoritmo 1. Pode-se mostrar que a transformação $\mathbf{Z}=\mathbf{W X}$ não altera a informação que os atributos fornecem sobre os rótulos, ou seja

$$
I(Y ; \mathbf{Z})=I(Y ; \mathbf{X})
$$

A informação mútua $I(Y ; \mathbf{X})$ é expressa por

$$
I(Y ; \mathbf{X})=H(\mathbf{X})-H(\mathbf{X} \mid Y)
$$

Do mesmo modo, $I(Y ; \mathbf{Z})$ é dada pela expressão

$$
I(Y ; \mathbf{Z})=H(\mathbf{Z})-H(\mathbf{Z} \mid Y)
$$

Mas como a transformação não altera a entropia conjunta (equação 5.4 nem a entropia conjunta condicional (equação 5.9), não é difícil ver que a informação fornecida pelos atributos sobre o rótulo também se conserva, resultando na Equação 5.12 .

O Algoritmo 2 realiza a minimização da multi-informação condicional. O 
algoritmo recebe como entrada um conjunto de treinamento formado por uma sequência

$$
(\mathbf{x}, y)^{(1)},(\mathbf{x}, y)^{(2)}, \ldots,(\mathbf{x}, y)^{(L)}
$$

em que $(\mathbf{x}, y)^{(l)}$ corresponde a um realização de um $\operatorname{par}(\mathbf{X}, Y)$, onde $\mathbf{X} \in\{0,1\}^{n}$ é um vetor de atributos e $Y \in\{0,1\}$ é um rótulo binário. $\mathrm{O}$ algoritmo produz uma matriz $n \times n$ binária $\mathbf{W}$ e uma sequência

$$
\mathbf{z}^{(1)}, \mathbf{z}^{(2)}, \ldots, \mathbf{z}^{(L)}
$$

em que $\mathbf{z}^{(l)}=\mathbf{W} \mathbf{x}^{(l)}$ e cada $\mathbf{z}^{(l)}$ pode ser entendido como uma realização de um vetor de atributos transformados $\mathbf{Z} \in\{0,1\}^{n}$.

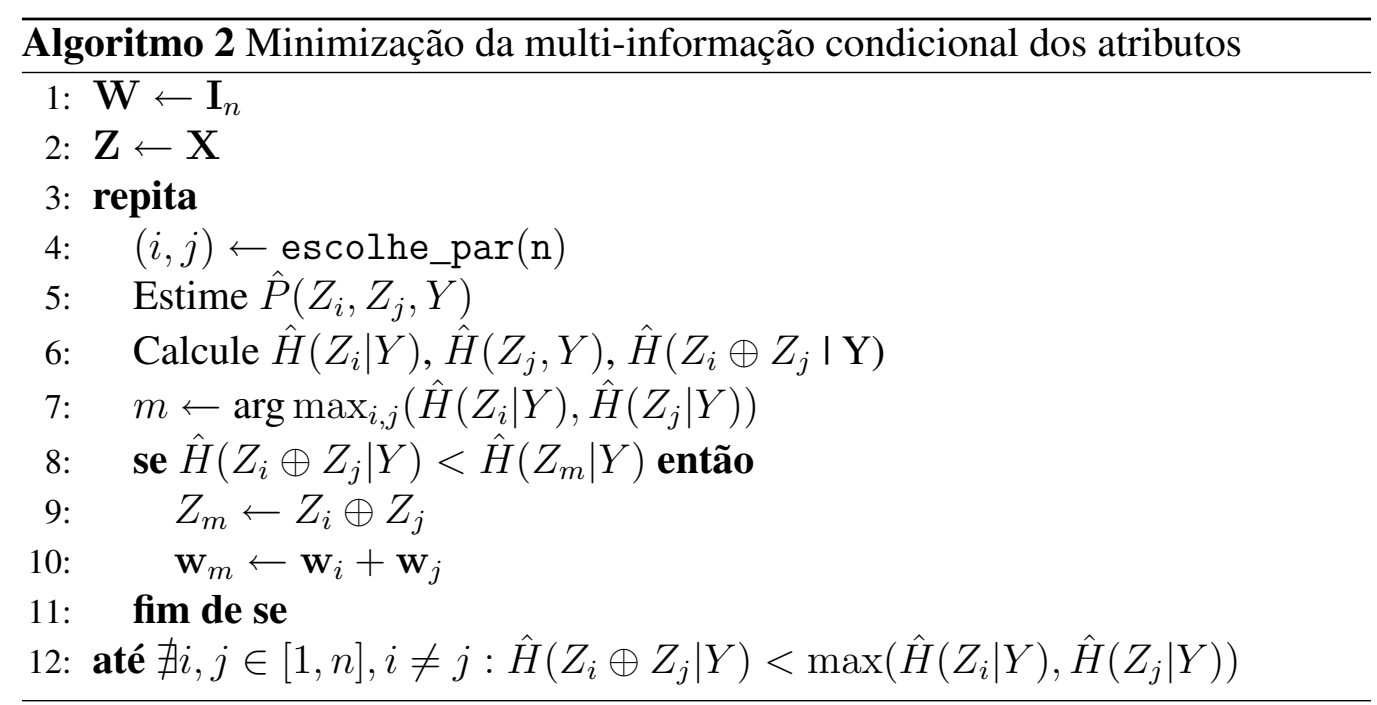

Assim como no Algoritmo 1, inicialmente a matriz de separação $\mathbf{W}$ é igual à matriz identidade, logo temos $\mathbf{Z}=\mathbf{X}$. Um par diferente de índices $(i, j)$ é escolhido a cada iteração. Estima-se em seguida a distribuição $\hat{P}\left(Z_{i}, Z_{j}, Y\right)$ por meio de um histograma. Em seguida as entropias condicionais $H\left(X_{i} \mid Y\right), H\left(X_{j} \mid Y\right)$ e $H\left(X_{i} \oplus X_{j} \mid Y\right)$ são calculadas. Caso $H\left(X_{i} \oplus X_{j} \mid Y\right)$ seja menor que alguma das outras entropias condicionais, o algoritmo decide substituir o atributo de maior entropia condicional por $X_{i} \oplus X_{j}$. Desse modo, a somatória das entropias condicionais (e, por consequência, a multi-informação condicional) é uma função decrescente ao longo da execução do algoritmo. Quando essa transformação de atributos ocorre, o algoritmo também atualiza a matriz $\mathbf{W}$, trocando a linha correspondente 


\subsection{CONVERGÊNCIA, COMPLEXIDADE E QUALIDADE DA SOLUÇÃO73}

à variável substituída pela soma (módulo-2) das duas linhas envolvidas nessa iteração, dado pela expressão $\mathbf{w}_{i}+\mathbf{w}_{j}$. Caso contrário, nenhuma transformação é aplicada nessa iteração e o algoritmo simplesmente continua em uma nova iteração, com outro par de índices. O processo é repetido até que não existam mais índices $(i, j)$ tal que a transformação de par de variáveis seja possível, ou seja, o algoritmo para quando qualquer transformação de par de variáveis resultaria em aumento das entropias condicionais marginais.

\subsection{Convergência, complexidade e qualidade da solução}

Sendo o Algoritmo 1 em muito similar ao Algoritmo 2, são válidas aqui muitas das considerações feitas a respeito daquele algoritmo na Seção 4.1. Em primeiro lugar, pode-se afirmar que o Algoritmo 2 obrigatoriamente converge em algum ponto, pois a multi-informação condicional somente decresce ao longo da execução e em algum momento o algoritmo vai atingir uma matriz $\mathbf{W}$ tal que a multiinformação não possa ser reduzida pelo tipo de atualização realizada durante uma iteração do algoritmo. Novamente, a solução encontrada pelo Algoritmo 1 não é única, pois qualquer permutação das linhas de $\mathbf{W}$ resultará em outro vetor $\mathbf{Z}$ tal que a soma das entropias condicionais se mantenha a mesma.

A complexidade do Algoritmo 2 é a mesma do Algoritmo 1, pois ambos seguem passos quase idênticos, com pequenas diferenças devidas ao tratamento dos rótulos, adicionados no presente caso. Assim como ocorreu com o Algoritmo 1 . observou-se empiricamente que o Algoritmo 2 converge para uma solução em número de iterações $O\left(n^{2}\right)$. Como o custo de cada iteração é dominado pelo custo de percorrer os $L$ exemplos de treinamento, pode-se afirmar que o tempo médio de execução do Algoritmo 2 é $O\left(n^{2} L\right)$. Por fim, também não se pode garantir que Algoritmo 2 produza a solução ótima (com menor multi-informação condicional possível) pois o seu modo de operação utiliza somente as distribuições marginais do tipo $P\left(Z_{i} \mid Y\right)$ e o conjunto destas não fornece um número de parâmetros suficientes para assegurar uma transformação ótima global sobre $P\left(Z_{1}, Z_{2}, \ldots, Z_{n}\right)$, conforme visto na Seção 4.1 . 


\subsection{Experimentos com imagens binárias}

O Algoritmo 2 foi aplicado a um conjunto de imagens binárias, visando visualizar os conjuntos de pixels decorrentes do agrupamento de variáveis realizado pelo algoritmo. A formação do conjunto de treinamento ocorreu do seguinte modo: parte-se de pares de (imagem de entrada, imagem desejada), como os pares mostrados na Figura 5.1 para uma tarefa de reconhecimento de textura e na Figura 5.2 para uma tarefa de reconhecimento de regiões contendo texto. Escolhe-se então uma janela retangular $W$ para realizar a amostragem desses pares de imagens. Seja $p$ uma determinada localidade da imagem desejada, isto é, $p$ é um pixel determinado por um par de coordenadas (largura, altura). A janela $W$ é posicionada sobre a imagem de entrada, com seu centro deslocado até as coordenadas de $p$. Nesse instante, $W$ determina uma região da imagem de entrada na vizinhança do ponto $p$. O conjunto de pixels cobertos pela janela determina uma realização do vetor de atributos $\mathbf{X}$. Ao mesmo tempo, o valor do pixel em $p$ da imagem desejada é registrado, fornecendo o valor do rótulo $Y$. Esse processo é ilustrado pela Figura 5.3. Esse par $(\mathbf{X}, Y)$ é adicionado ao conjunto de treinamento. Para transformar os pedaços de imagens amostrados por meio da janela em vetores, os pontos de cada pedaço de imagem são percorridos em ordem lexicográfica, dando origem aos elementos do vetor (ver Figura 5.4). Esse procedimento é repetido para todas as localidades $p$ da imagem desejada (excetuando-se as bordas, para que a janela $W$ esteja sempre totalmente contida pela imagem de entrada), gerando o conjunto de treinamento completo.

O algoritmo é aplicado em seguida sobre o conjunto de treinamento, dando origem à matriz W. Cada linha dessa matriz corresponde a um grupo de atributos. Cada linha é novamente disposta como uma imagem, seguindo o processo da Figura 5.4 dando origem a subjanelas da janela original $W$. O algoritmo foi aplicado duas vezes, a primeira para a tarefa de reconhecimento de texturas e a segunda para a tarefa de reconhecimento de regiões de texto. No caso do reconhecimento de texto, foram usados três pares de treinamento como os da Figura 5.1 e a amostragem foi realizada com uma janela de 5 pixels de altura por 7 pixels de largura. Para a tarefa de reconhecimento de regiões de texto, foram utilizados dois pares de imagens como as da Figura 5.2, também com uma janela de 


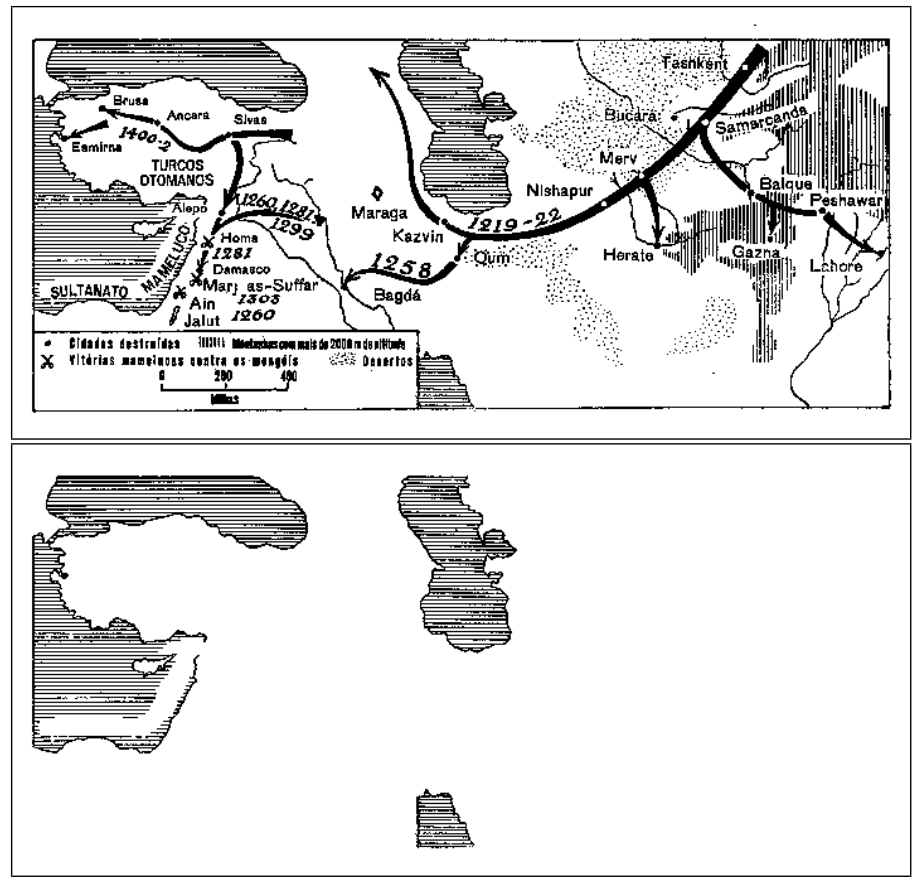

Figura 5.1: Imagem de entrada (acima) e imagem desejada (abaixo) para uma tarefa de reconhecimento de texturas em imagens.

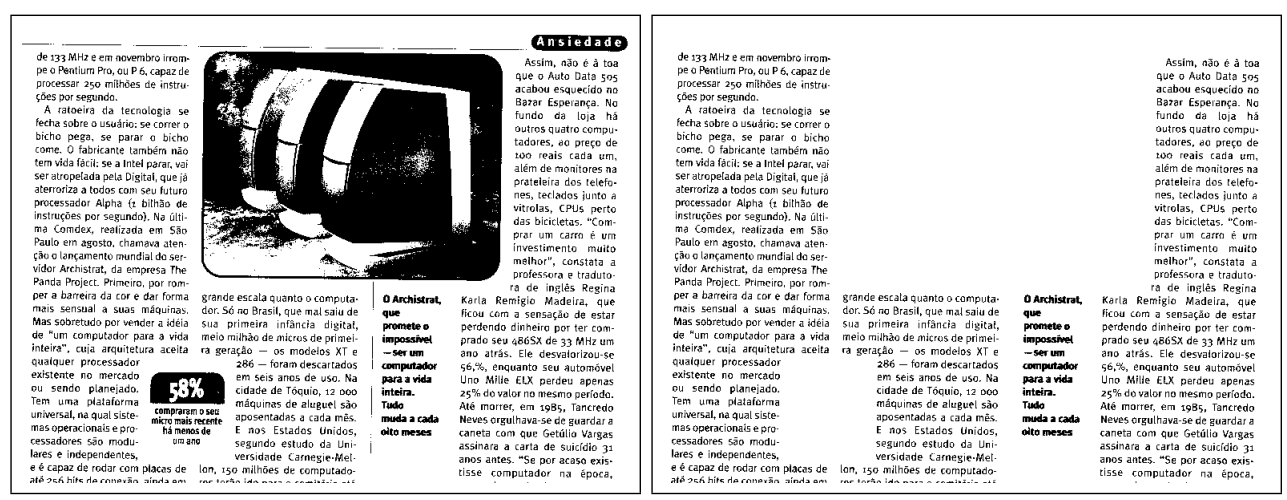

Figura 5.2: Imagem de entrada (esquerda) e imagem desejada (direita) para uma tarefa de reconhecimento de regiões com texto em imagens.

tamanho 5x7. Esses dois conjuntos de imagens serão novamente utilizados em experimentos nos capítulos 6 e 7. Os resultados foram qualitativamente iguais para as duas tarefas, de modo que somente serão mostradas as subjanelas obtidas para reconhecimento de texturas. Observa-se, na Figura 5.5, que os pixels agrupam-se de acordo com estruturas reconhecíveis na imagem de entrada, como 


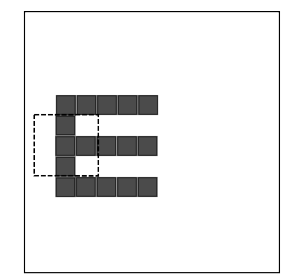

imagem de entrada

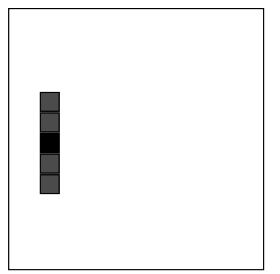

imagem desejada

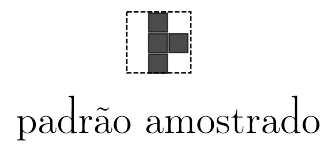

rótulo

Figura 5.3: Ilustração de amostragem. No caso, uma janela $3 \times 3$ é deslocada sobre a imagem de entrada. O padrão visto através da janela é registrado. Ao mesmo tempo, o valor correspondente na imagem desejada fornece o valor do rótulo. O par (padrão de entrada, rótulo) é adicionado ao conjunto de treinamento.

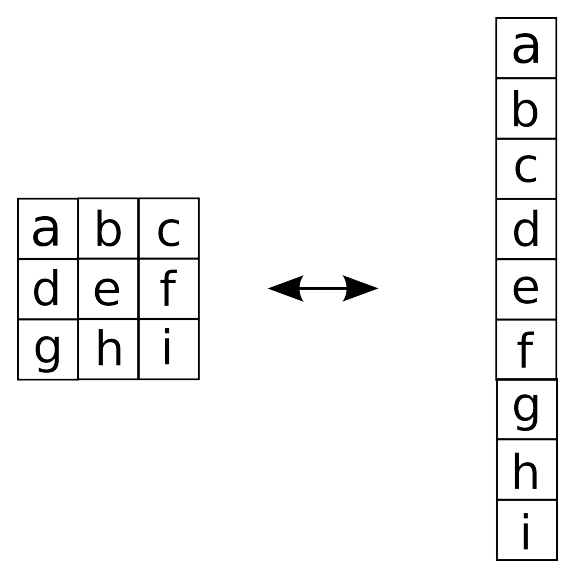

Figura 5.4: Transformação de pedaço de imagem (visto por uma janela $3 \times 3$ ) em um vetor. Os elementos do vetor são preenchidos pelos pontos da janela, em ordem lexicográfica. O processo inverso pode ser feito para restaurar pedaços de imagens ou janelas a partir de vetores.

linhas horizontais, verticais, às vezes formando pares de linhas tais como aparecem nas texturas da imagem de entrada. No entanto, nenhum desses grupos é muito grande, a maior parte deles não tem mais do que 4 pixels. No Algoritmo 2 os grupos crescem enquanto for possível diminuir a multi-informação condicional dos atributos $M I(\mathbf{Z} \mid Y)$, onde os atributos são funções de paridade calculadas 
sobre cada grupo de pixels. Uma vez que a multi-informação condicional mede a redundância residual entre os atributos após o conhecimento do valor do rótulo $Y$, o comportamento observado pode indicar que a observação do pixel de saída elimina parte considerável da redundância entre pixels de entrada, desse modo os grupos de pixels não podem crescer muito.

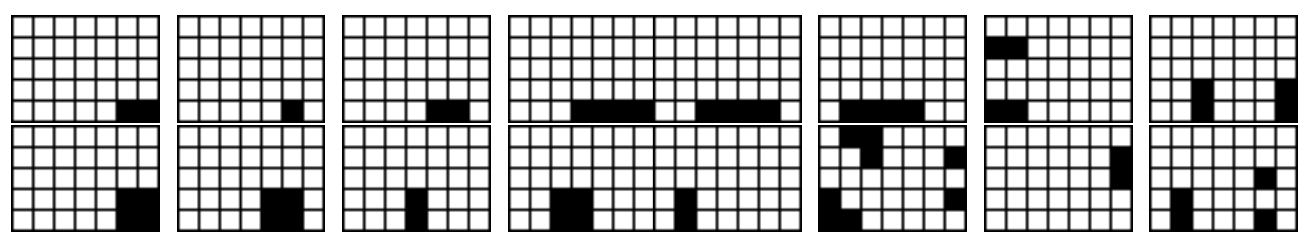

Figura 5.5: Algumas subjanelas obtidas com o Algoritmo2 para a tarefa de segmentação de textura.

A seguir serão apresentados dois cenários onde pode ser útil um algoritmo para reduzir a multi-informação condicional de variáveis aleatórias.

Considerando o problema de seleção de atributos, Krause e Guéstrin mostraram [KG05] que a independência condicional entre atributos permite a um algoritmo guloso obter desempenho quase ótimo, quando a função a ser maximizada é a informação mútua entre atributos selecionados e rótulos

$$
I\left(Y ;\left(X_{1}, X_{2}, \ldots, X_{m}\right)\right) .
$$

Nesse contexto, a minimização da multi-informação condicional dos atributos antes da seleção pode ser benéfica. Outra situação em que a independência condicional é desejável é no aprendizado de modelos probabilísticos, pois contribui para a obtenção de modelos mais simples. Por exemplo, para três variáveis aleatórias $\left(X_{1}, X_{2}, X_{3}\right)$, caso $X_{2}$ e $X_{3}$ sejam independentes dado $X_{1}$ a distribuição conjunta pode ser escrita como

$$
P\left(X_{1}, X_{2}, X_{3}\right)=P\left(X_{1}\right) P\left(X_{2} \mid X_{1}\right) P\left(X_{3} \mid X_{1}\right)
$$

A forma fatorada acima torna o modelo mais simples, eliminando parâmetros que relacionem $X_{2}$ e $X_{3}$, além de diminuir a complexidade do cálculo de distribuições marginais [Jor04]. 


\section{Capítulo 6}

\section{Construção de Atributos Baseada em Aná- lise de Interações}

Neste capítulo será apresentado um método para construção de atributos binários em problemas de classificação baseado na manipulação de valores de Informação de Interação, publicado inicialmente em [SHHJ07]. Assim como os métodos apresentados nos Capítulos $4 \mathrm{e}$ 5, o método introduzido aqui também usa uma matriz de cálculo de paridade para transformar um vetor binário. O objetivo da transformação é reduzir a magnitude das interações entre os atributos. A seguir será apresentado o mecanismo básico de manipulação da informação de interação de um par de atributos. Logo após, será justificado o interesse na manipulação dos valores de interação.

\subsection{Transformações de atributos binários e informação de in- teração}

Sejam $X_{i}, X_{j}$ dois atributos binários e $Y \in\{0,1\}$ um rótulo. Considerando uma transformação elementar

$$
\left(Z_{i}, Z_{j}\right)=\left(X_{i}, X_{i} \oplus X_{j}\right)
$$

vamos estudar agora o efeito dessa transformação sobre a informação de interação, ou seja, a relação entre $I\left(Y ; Z_{i} ; Z_{j}\right)$ e $I\left(Y ; X_{i} ; X_{j}\right)$. Da definição de informação de interação segue que

$$
I\left(Y ; Z_{i} ; Z_{j}\right)=I\left(Y ;\left(Z_{i}, Z_{j}\right)\right)-I\left(Y ; Z_{i}\right)-I\left(Y ; Z_{j}\right)
$$


e

$$
I\left(Y ; X_{i} ; X_{j}\right)=I\left(Y ;\left(X_{i}, X_{j}\right)\right)-I\left(Y ; X_{i}\right)-I\left(Y ; X_{j}\right)
$$

Já foi mostrado (Equação 5.12) que esse tipo de transformação preserva a informação que os atributos fornecem sobre o rótulo:

$$
I\left(Y ;\left(Z_{i}, Z_{j}\right)\right)=I\left(Y ;\left(X_{i}, X_{j}\right)\right)
$$

Substituindo 6.3) em 6.1 temos

$$
I\left(Y ; Z_{i} ; Z_{j}\right)=I\left(Y ;\left(X_{i}, X_{j}\right)\right)-I\left(Y ; Z_{i}\right)-I\left(Y ; Z_{j}\right)
$$

Subtraindo6.2 de 6.4 obtém-se:

$$
I\left(Y ; Z_{i} ; Z_{j}\right)-I\left(Y ; X_{i} ; X_{j}\right)=I\left(Y ; X_{i}\right)+I\left(Y ; X_{j}\right)-I\left(Y ; Z_{i}\right)-I\left(Y ; Z_{j}\right)
$$

A expressão (6.5) provê um meio de manipularmos os valores de informação de interação de um par de atributos (com relação ao rótulo). No nosso caso particular, $Z_{i}=X_{i}$, portanto $I\left(Y ; Z_{i}\right)=I\left(Y ; X_{i}\right)$, simplificando a expressão 6.5 para

$$
I\left(Y ; Z_{i} ; Z_{j}\right)-I\left(Y ; X_{i} ; X_{j}\right)=I\left(Y ; X_{j}\right)-I\left(Y ; Z_{j}\right)
$$

Diferentemente do que ocorre com a multi-informação, minimizar (ou maximizar) a informação de interação envolvendo dois atributos não equivale a minimizar (ou maximizar) uma função global sobre todos os atributos. A seguir, argumentamos em favor de um método empírico para reduzir a magnitude das interações entre pares de atributos, apesar do método não poder ser associado a uma medida global de qualidade dos mesmos.

Examinemos o caso em que $I\left(Y ; X_{i} ; X_{j}\right)>0$. Isso significa que a observação conjunta do par $\left(X_{i}, X_{j}\right)$ origina informação adicional sobre o rótulo que não pode ser depreendida da observação isolada de $X_{i}$ ou $X_{j}$. Para que um modelo estatístico conseguisse tirar proveito dessa informação seria necessário que o modelo permitisse a inclusão de um parâmetro que refletisse essa interação entre atributos. Isso não ocorre, por exemplo, com o classificador Naive Bayes ou em modelos lineares simples. Mesmo quando possível, a inclusão de um parâmetro 
de interação no modelo acarreta aumento da complexidade do mesmo, possivelmente diminuindo a precisão estatística no aprendizado do modelo. A alternativa investigada aqui é aplicar uma transformação aos dados que diminua a magnitude da interação positiva. Recorrendo à expressão (6.6), vemos que

$$
I\left(Y ; X_{j}\right)<I\left(Y ; Z_{j}\right)
$$

implica

$$
I\left(Y ; Z_{i} ; Z_{j}\right)<I\left(Y ; X_{i} ; X_{j}\right)
$$

Como a informação total se mantém constante, ou seja

$$
I\left(Y ;\left(X_{i}, X_{j}\right)\right)=I\left(Y ;\left(Z_{i}, Z_{j}\right)\right)
$$

a interação positiva diminui de magnitude porque a informação que era criada pela observação conjunta de $\left(X_{i}, X_{j}\right)$ é transferida para o novo atributo $Z_{j}$.

Analisemos agora a situação contrária, em que $I\left(Y ; X_{i} ; X_{j}\right)<0$, equivalente a dizer

$$
I\left(Y ;\left(X_{i}, X_{j}\right)\right)<I\left(Y ; X_{i}\right)+I\left(Y ; X_{j}\right) .
$$

Isso significa que há certa redundância entre $I\left(Y ; X_{i}\right)$ e $I\left(Y ; X_{j}\right)$ no que diz respeito à predição do rótulo. Ambos atributos contém a mesma informação sobre $Y$. Nesse caso, um modelo construído sobre esses atributos necessita levar em conta a interação entre $X_{i}$ e $X_{j}$ a fim de não contar duplamente essa evidência. Em outras palavras, supondo que a observação de $X_{i}$ favoreça o valor $Y=y$ então a observação de $X_{j}$ também favorecerá o mesmo valor e um modelo que ignore a interação contará duas vezes a evidência em favor de $Y=y$. Um recurso nesse caso é tentar diminuir a redundância entre os atributos, ou seja, tornar a informação de interação menos negativa. A Equação (6.6) nos informa que para termos

$$
I\left(Y ; Z_{i} ; Z_{j}\right)>I\left(Y ; X_{i} ; X_{j}\right)
$$

é preciso que

$$
I\left(Y ; X_{j}\right)>I\left(Y ; Z_{j}\right)
$$

A redundância entre os atributos é diminuída porque o novo atributo $Z_{j}$ perde 
parte da informação compartilhada por $X_{i}$ e $X_{j}$.

Do acima exposto, concluímos que controlar o valor da informação de interação favorece modelos mais simples, que contenham um menor número de parâmetros/termos responsáveis por modelar explicitamente as interações entre atributos. Para projetarmos um método iterativo de redução das interações é preciso avaliar o impacto que a redução da informação de interação entre um determinado par de atributos terá sobre o valor da interação com os demais. Suponha que originalmente tenhamos uma tripla de atributos $\left(X_{i}, X_{j}, X_{k}\right)$. A transformação resulta em uma nova tripla $\left(Z_{i}, Z_{j}, X_{k}\right)$. Como $X_{i}=Z_{i}$, nos interessa apenas estudar $I\left(Y ; X_{j} ; X_{k}\right)$ com relação a $I\left(Y ; Z_{j} ; X_{k}\right)$. Esses dois valores de interação são dados por

$$
I\left(Y ; X_{j} ; X_{k}\right)=I\left(Y ;\left(X_{j}, X_{k}\right)\right)-I\left(Y ; X_{j}\right)-I\left(Y ; X_{k}\right)
$$

$\mathrm{e}$

$$
I\left(Y ; Z_{j} ; X_{k}\right)=I\left(Y ;\left(Z_{j}, X_{k}\right)\right)-I\left(Y ; Z_{j}\right)-I\left(Y ; X_{k}\right) .
$$

Subtraindo uma equação da outra temos

$I\left(Y ; Z_{j} ; X_{k}\right)-I\left(Y ; X_{j} ; X_{k}\right)=I\left(Y ;\left(Z_{j}, X_{k}\right)\right)-I\left(Y ;\left(X_{j}, X_{k}\right)\right)+I\left(Y ; X_{j}\right)-I\left(Y ; Z_{j}\right)$.

Note-se que nessa situação não podemos afirmar que $I\left(Y ;\left(Z_{j}, X_{k}\right)\right)$ seja igual a $I\left(Y ;\left(X_{j}, X_{k}\right)\right)$.

Em princípio, caracterizar o comportamento da informação de interação $I\left(Y ; Z_{j} ; X_{k}\right)$ exigiria conhecer a distribuição $P\left(Y, Z_{j}, X_{k}\right)$, mas pode-se apontar tendências conforme a variação de $I\left(Y ; Z_{j}\right)$. Quando queremos tornar a interação $I\left(Y ; Z_{i} ; Z_{j}\right)$ menos positiva, o valor $I\left(Y ; Z_{j}\right)$ é aumentado com relação a $I\left(Y ; X_{j}\right)$. O valor $I\left(Y ;\left(Z_{j}, X_{k}\right)\right)$ pode aumentar na mesma medida em relação a $I\left(Y ;\left(X_{j}, X_{k}\right)\right)$ ou tender a ficar estável (se a nova informação acrescentada a $Z_{j}$ for redundante com a informação já contida em $X_{k}$ ), mas não pode diminuir. Desse modo, a tendência é que $I\left(Y ; Z_{j} ; X_{k}\right)$ acompanhe $I\left(Y ; Z_{i} ; Z_{j}\right)$ e também diminua.

Por outro lado, para tornar $I\left(Y ; Z_{i} ; Z_{j}\right)$ menos negativa, o valor $I\left(Y ; Z_{j}\right)$ é diminuído. Então $I\left(Y ;\left(Z_{j}, X_{k}\right)\right)$ pode acompanhar a diminuição ou continuar com o mesmo valor, se a informação retirada de $Z_{j}$ também estiver contida em $X_{k}$. 


\begin{tabular}{|l|c|c|}
\hline$I\left(Y ; Z_{i} ; Z_{j}\right)-I\left(Y ; X_{i} ; X_{j}\right)$ & $\delta$ & $-\delta$ \\
\hline$I\left(Y ; Z_{j}\right)-I\left(Y ; X_{j}\right)$ & $-\delta$ & $\delta$ \\
\hline$I\left(Y ;\left(Z_{j} ; X_{k}\right)\right)-I\left(Y ;\left(X_{j} ; X_{k}\right)\right)$ & {$[-\delta, 0]$} & {$[0, \delta]$} \\
\hline$I\left(Y ; Z_{j} ; X_{k}\right)-I\left(Y ; X_{j} ; X_{k}\right)$ & {$[0, \delta]$} & {$[-\delta, 0]$} \\
\hline
\end{tabular}

\section{Tabela 6.1:}

Variação da informação de interação $I\left(Y ; Z_{i} ; Z_{j}\right)-I\left(Y ; X_{i} ; X_{j}\right)$ e seu efeito em $I\left(Y ; Z_{j} ; X_{k}\right)-I\left(Y ; X_{j} ; X_{k}\right)$.

Assim, $I\left(Y ; Z_{i} ; X_{k}\right)$ tende a ficar menos negativa também. Em resumo, quando um atributo novo $Z_{i}$ é criado para diminuir a interação positiva com um segundo atributo $Z_{j}$, a interação de $Z_{j}$ com os demais atributos tende a diminuir também. E quando a transformação é aplicada para eliminar a redundância de $Z_{j} \operatorname{com} Z_{i}$ (ou seja, para tornar a informação de interação menos negativa), a tendência é que a redundância entre $Z_{j}$ e o restante dos atributos também diminua. $\mathrm{O}$ raciocínio exposto acima é resumido na Tabela 6.1. Desse modo, justifica-se a esperança que a transformação envolvendo um par de variáveis também aproxime de zero os valores das interações com o restante dos atributos. Uma analogia pode ser traçada entre os objetivos da ICA e do método esboçado acima. Em ICA o objetivo é reduzir a informação mútua entre os atributos. No método discutido aqui o objetivo é reduzir informação compartilhada entre atributos a respeito do rótulo. Idealmente, isso se refletiria numa situação em que: i) toda a informação sobre o rótulo estivesse contida em algum atributo, nenhuma informação nova seria depreendida da observação de conjuntos maiores de atributos; ii) não existisse redundância entre atributos quanto ao rótulo; toda informação contida por um atributo pertenceria somente a este atributo. Na situação descrita os atributos atuariam como blocos básicos de informação sobre o rótulo.

\subsection{Algoritmo para redução de interações}

Um algoritmo iterativo para redução da magnitude da informação de interação entre pares de atributos é apresentado a seguir. O Algoritmo 3 tem basicamente a mesma estrutura dos algoritmos 1 e 2. A entrada do algoritmo consiste numa sequência de tamanho $L$ de pares $(\mathbf{x}, y)$ em que $\mathbf{x}$ é uma realização de um vetor aleatório $\mathbf{X} \in\{0,1\}^{n}$ e $y$ é a realização de uma variável aleatória $Y \in\{0,1\}$ 
que atua como rótulo. Também deve ser fornecido como entrada um parâmetro MAX_ITER que determina o número máximo de iterações que o algoritmo deve realizar, pois não se pode garantir sua convergência para uma solução fixa. O algoritmo devolve uma matriz $\mathbf{W}$ que caracteriza uma transformação $\mathbf{Z}=\mathbf{W X}$ e também uma sequência de vetores de atributos pós transformação

$$
\mathbf{z}^{(1)}, \mathbf{z}^{(2)}, \ldots, \mathbf{z}^{(L)}
$$

em que $\mathbf{z}^{(l)}=\mathbf{W} \mathbf{x}^{(l)}$. Inicialmente a matriz $\mathbf{W}$ é igual à matriz identidade $\mathrm{e}$ os atributos transformados $\mathbf{Z}$ são iguais aos atributos originais $\mathbf{X}$. $\mathrm{O}$ algoritmo mantém um contador de iterações, que é zerado inicialmente.

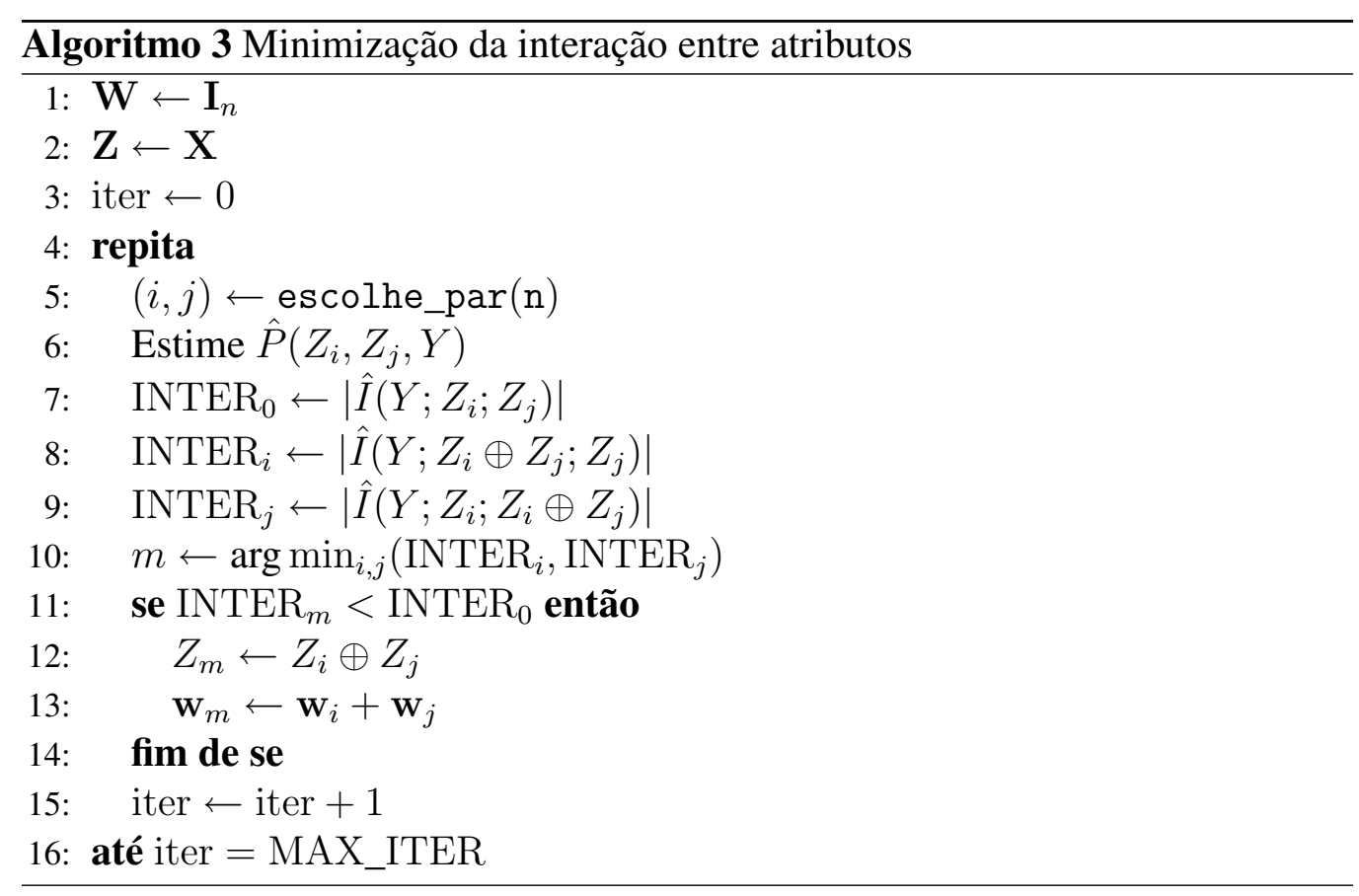

Em cada passo do processo iterativo, o algoritmo seleciona um par de índices distintos $(i, j)$ e estima a probabilidade conjunta $\hat{P}\left(Z_{i}, Z_{j}, Y\right)$. Em seguida o algoritmo calcula três valores de informação de iteração, correspondentes ao par de atributos originais:

$$
\hat{I}\left(Y ; Z_{i} ; Z_{j}\right)
$$


e a cada uma das transformações possíveis de ser aplicada nesse passo:

$$
\hat{I}\left(Y ; Z_{i} \oplus Z_{j} ; Z_{j}\right)
$$

e

$$
\hat{I}\left(Y ; Z_{i} ; Z_{i} \oplus Z_{j}\right)
$$

O objetivo é determinar o par de atributos que resulte na menor informação de interação em valor absoluto, i.e. aquele mais próximo de zero. Caso seja necessário aplicar uma transformação nesse passo, o algoritmo seleciona o índice adequado, atualiza a matriz $\mathbf{W}$ e também os atributos. Caso contrário, o algoritmo prossegue na próxima iteração. Ao fim de cada passo, o algoritmo incrementa o contador de iterações e verifica se deve parar a execução, comparando o contador com o parâmetro de entrada MAX_ITER. Como exposto anteriormente, não é possível afirmar que o algoritmo convirja para uma solução, em princípio o algoritmo poderia executar indefinidamente e sempre encontrar um par de atributos $\left(Z_{i}, Z_{j}\right)$ cuja informação de interação $I\left(Y ; Z_{i} ; Z_{j}\right)$ pudesse ser reduzida (em módulo). Desse modo, o número de iterações que o algoritmo executa é controlado pelo parâmetro MAX_ITER. O tempo de execução de cada iteração é dominado pela tarefa de percorrer os $L$ exemplos para estimar a probabilidade $\hat{P}\left(Z_{i}, Z_{j}, Y\right)$. Assim, a complexidade total do algoritmo é $O\left(\mathrm{MAX} \_\right.$ITER $\left.\cdot L\right)$.

\subsection{Experimentos}

Para testar a capacidade do Algoritmo 3 de reduzir as interações entre atributos foram realizados alguns experimentos envolvendo imagens binárias. A motivação para explorar o Algoritmo 3 com imagens binárias vem do problema do projeto de operadores em dois níveis para esse tipo de imagem, a aplicação do nesse contexto será detalhada no Capítulo 7. O intuito dos experimentos apresentados nesta seção é verificar se o processo iterativo descrito é capaz realmente de criar atributos com interação reduzida, corroborando a análise do algoritmo apresentada acima. Para isso os valores de interação pré e pós transformação foram medidos, por meio de quatro índices (nas fórmulas abaixo, o símbolo $K$ denota o número de pares distintos $(i, j), K=n(n-1) / 2)$ : 
- Interação máxima

$$
I_{\max }=\max _{i, j} I\left(Y ; \theta_{i} ; \theta_{j}\right), i \neq j
$$

- Interação mínima

$$
I_{\text {min }}=\min _{i, j} I\left(Y ; \theta_{i} ; \theta_{j}\right), i \neq j
$$

- Média do valor absoluto de interação

$$
\mu(\mathrm{abs})=\frac{\sum_{i \neq j}\left|I\left(Y ; \theta_{i} ; \theta_{j}\right)\right|}{K}
$$

- Desvio padrão do valor absoluto de interação

$$
\text { d.p. }(\mathrm{abs})=\sqrt{\frac{\sum_{i \neq j} I\left(Y ; \theta_{i} ; \theta_{j}\right)^{2}}{K}-\mu(\mathrm{abs})^{2}}
$$

Para o cálculo dos valores pré-transformação $\theta_{i}$ é substituído por $X_{i}$. Para avaliação de valores pós-transformação, $\theta_{i}$ é substituído por $Z_{i}$. A aplicação do Algoritmo 3 sobre imagens binárias seguiu os mesmos passos usados na seção 5.3 para os experimentos com o Algoritmo 2, Para imagens binárias, os atributos $X_{i}$ correspondem a pixels e os atributos $Z_{i}$ correspondem a funções de paridade calculadas sobre subconjuntos de pixels. O Algoritmo 3 foi aplicado em três conjuntos distintos de imagens, relacionados a três tarefas diferentes. Dois desses conjuntos já foram apresentados na Seção 5.3. O primeiro conjunto era formado por 3 pares (imagem de entrada, imagem desejada), em uma tarefa de reconhecimento de regiões de texto em páginas digitalizadas de revistas (ver Figura 5.2). Esse conjunto foi amostrado com uma janela quadrada com 11 pixels de lado. $\mathrm{O}$ segundo conjunto era formado por dois pares de imagens de treinamento em uma tarefa de reconhecimento de texturas (ver Figura 5.1). A amostragem foi realizada com janela quadrada de 9 pixels de lado. O terceiro conjunto vem de uma tarefa de reconhecimento de caracteres em páginas digitalizadas de um livro (nesse caso, queremos reconhecer a letra "s"). Foram utilizados 6 pares de imagens de trei- 
namento, como aquele mostrado na Figura 6.1. A amostragem foi realizada por janela com 7 pixels de largura e 9 pixels de altura.

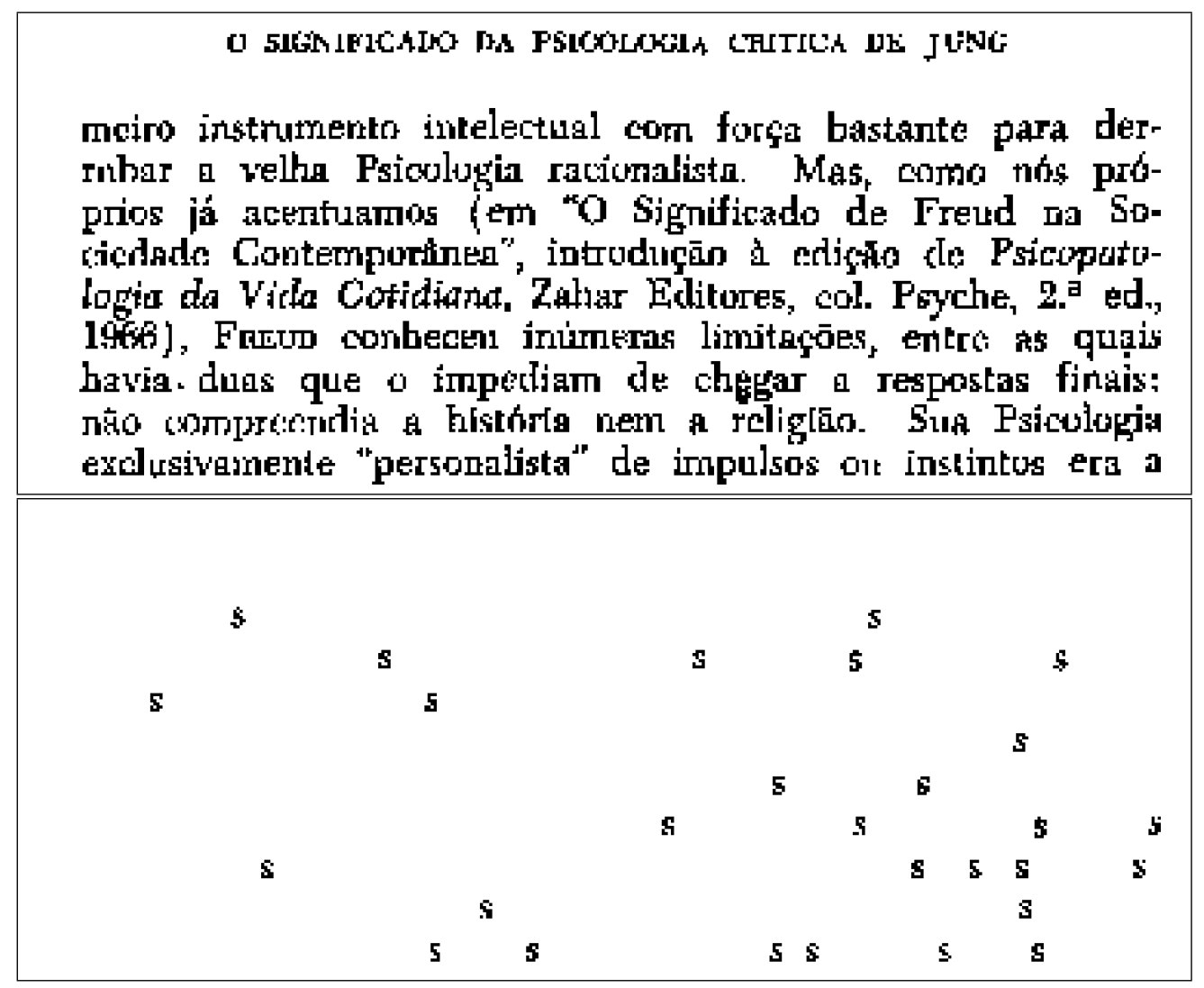

Figura 6.1: Imagem de entrada e imagem desejada para uma tarefa de reconhecimento de caractere.

\begin{tabular}{|l|c|c|c|c|}
\cline { 2 - 5 } \multicolumn{1}{c|}{} & \multicolumn{4}{c|}{ Informação de Interação (bits) } \\
\cline { 2 - 5 } \multicolumn{1}{c|}{} & $I_{\max }$ & $I_{\min }$ & $\mu($ abs $)$ & d.p.(abs) \\
\hline Pré-transformação & 0.029 & -0.304 & 0.100 & 0.052 \\
\hline Pós-transformação & 0.051 & -0.027 & 0.004 & 0.002 \\
\hline
\end{tabular}

Tabela 6.2: Medidas de interação pré e pós transformação para as imagens de reconhecimento de áreas de texto. Os valores pré-transformação são medidos diretamente sobre os pixels da imagem. Os valores pós-transformação se referem às funções de paridade calculadas sobre os grupos de pixels encontrados pelo Algoritmo 3 .

Os resultados das medidas de interação são mostrados na Tabela 6.2 para reconhecimento de texto, Tabela 6.3 para reconhecimento de textura e Tabela 6.4 
para reconhecimento de caractere. Nos três casos, tanto o valor médio da magnitude das interações quanto o desvio padrão tiveram redução significativa. Isso mostra que o método proposto foi eficaz, causando uma concentração dos valores de interação em torno de zero. Quando comparamos os valores máximos e mínimos de interação, o comportamento não é tão consistente. Para o primeiro conjunto de dados o valor mínimo de interação se aproximou bastante de zero (passando de -0.304 bits para -0.027 bits) enquanto o valor de interação máxima passou de 0.029 bits para 0.051 bits. Com o segundo conjunto de dados a interação mínima não se alterou significativamente (de -0.037 bits para -0.031 bits) enquanto a interação máxima reduziu-se de 0.163 bits para 0.038 bits. $\mathrm{O}$ mesmo quadro se repete para o terceiro conjunto de dados, em que a interação mínima passou de -0.0018 para -0.0022 , enquanto a interação máxima passou de 0.0136 para 0.0034 . Isso mostra que a redução de interações realizada pelo algoritmo não ocorre de maneira totalmente uniforme, podendo existir alguns casos em que a interação é reforçada.

\begin{tabular}{|l|c|c|c|c|}
\cline { 2 - 5 } \multicolumn{1}{c|}{} & \multicolumn{4}{c|}{ Informação de Interação (bits) } \\
\cline { 2 - 5 } \multicolumn{1}{c|}{} & $I_{\max }$ & $I_{\min }$ & $\mu($ abs $)$ & d.p.(abs) \\
\hline Pré-transformação & 0.163 & -0.037 & 0.014 & 0.020 \\
\hline Pós-transformação & 0.038 & -0.031 & 0.002 & 0.003 \\
\hline
\end{tabular}

Tabela 6.3: Medidas de interação pré e pós transformação para as imagens de reconhecimento de textura.

\begin{tabular}{|l|c|c|c|c|}
\cline { 2 - 5 } \multicolumn{1}{c|}{} & \multicolumn{4}{c|}{ Informação de Interação (bits) } \\
\cline { 2 - 5 } \multicolumn{1}{c|}{} & $I_{\max }$ & $I_{\min }$ & $\mu($ abs $)$ & d.p.(abs) \\
\hline Pré-transformação & 0.0136 & -0.0018 & 0.0010 & 0.0014 \\
\hline Pós-transformação & 0.0034 & -0.0022 & 0.0001 & 0.0002 \\
\hline
\end{tabular}

Tabela 6.4: Medidas de interação pré e pós transformação para as imagens de reconhecimento de caractere. 


\section{Capítulo 7}

\section{Projeto de filtros de imagens em dois níveis}

Este capítulo trata da aplicação do Algoritmo 4 ao problema do projeto de operadores de imagens binárias em dois níveis. Tais operadores se organizam da seguinte maneira: no primeiro nível, um conjunto de $k$ operadores é aplicado à imagem de entrada. Cada um desses operadores "enxerga" uma porção diferente da imagem de entrada, pois utilizam janelas diversas. As saídas desses operadores de primeiro nível são combinadas por um operador de segundo nível, que produz a imagem final. O projeto de tais operadores pode ser interpretado como um problema de combinação de classificadores, em que a estratégia para se conseguir a diversidade entre os classificadores é variar os atributos utilizados pelos classificadores/operadores no primeiro nível. Nesse contexto, o Algoritmo 4 é usado como mecanismo gerador de diversidade. A capacidade do algoritmo de agrupar atributos binários de acordo com a presença de interações é explorada para auxiliar a divisão da imagem em subjanelas.

\subsection{Operadores de imagens binárias}

Os operadores de imagens binárias discutidos neste capítulo pertencem à classe dos operadores invariantes à translação e localmente definidos. Essa classe é equivalente à classe das funções Booleanas [BB91]. A localidade decorre do fato do operador utilizar uma porção restrita da imagem, delimitada por uma janela. O nosso objetivo é projetar esses operadores automaticamente a partir de conjuntos de imagens de treinamento. O processo geral de projeto tratado aqui envolve estimar a probabilidade conjunta de pares (imagem de entrada, imagem desejada) a partir de uma amostra. Em seguida é aplicado um algoritmo de aprendizado para 
fornecer um operador que minimize uma medida de erro com relação à probabilidade estimada. A janela do operador desempenha um papel importante nesse processo pois é ela que determina a amostra usada no aprendizado. Uma janela grande tem a característica desejável de aumentar a informação utilizada pelo operador. Em contrapartida, o aumento do número de pixels ocasiona perda de precisão da estimativa da distribuição conjunta, além de problemas computacionais.

Algumas ideias já foram propostas para amenizar os problemas causados por janelas grandes. Uma abordagem comum é restringir o espaço de operadores [Dou92, [SDA98] ou ainda usar uma combinação sequencial de operadores, construída iterativamente [SDA99, DZC96, HDB00]. Outra proposta é empregar técnicas multirresolução, em que uma janela grande é decomposta em uma pirâmide de janelas empilhadas [VBH05].

Outra solução [Hir05] recente é empregar uma técnica de projeto em dois níveis, baseada no princípio da Generalização por Empilhamento (Stacked Generalization) [Wol92]. Nesse esquema, vários operadores (cada um empregando uma janela diferente) produzem um conjunto de saídas que são reunidas e passadas para um operador de segundo nível, que produz o valor de saída final. Como resultado, o operador de dois níveis consegue usar uma área maior da imagem, mesmo que indiretamente, aumentando o desempenho do operador e simultaneamente controlando fatores como o custo computacional e a precisão estatística [Hir05, Hir09]. Apesar de ser um avanço, o projeto em dois níveis ainda requer a escolha do número e da forma das subjanelas, um processo que pode ser bastante trabalhoso se realizado manualmente. Neste capítulo, um método para a automação desse processo será apresentado, originalmente proposto em [SHJ10]. Mas antes disso, o projeto de um operador de dois níveis será discutido em maior detalhe.

\subsection{Projeto de operadores em dois níveis}

Um operador binário, invariante à translação e definido localmente, denominado $\Psi$, pode ser expresso por

$$
[\Psi(S)](p)=\psi\left(S \cap W_{p}\right)
$$


em que $S$ é uma imagem binária, $p$ denota a localização de um pixel arbitrário da imagem, $W$ é uma janela e $\psi$ é uma função Booleana de $n=|W|$ variáveis. O argumento da função Booleana, $S \cap W_{p}$ corresponde a um vetor de tamanho $n$ composto pelos pixels da imagem $S$ nas vizinhanças de $p$ observados através de $W$.

Para caracterizar estatisticamente o par (imagem de entrada, imagem desejada), nós adotamos um modelo em que as imagens de entrada são geradas por um processo aleatório $\mathbf{U}$ e as imagens desejadas são geradas por um processo $\mathbf{V}$, sendo $\mathbf{U}$ e $\mathbf{V}$ conjuntamente estacionários. A estacionaridade permite tratar as observações locais feitas na vizinhança de $p$, usando a janela $W$, como realizações de um vetor aleatório $\mathbf{X}$. De maneira análoga, o valor do pixel da imagem desejada na localidade $p$ pode ser interpretado como a realização de uma variável aleatória $Y$. O par $(\mathbf{X}, Y)$ obedece à distribuição $P(\mathbf{X}, Y)$. Assim, pode-se definir o erro absoluto médio (Mean Absolute Error - MAE) de um operador $\Psi$ como

$$
\operatorname{MAE}(\Psi)=\sum_{\mathbf{x} \in \mathcal{X}} \sum_{y \in \mathcal{Y}} P(\mathbf{x}, y)|\psi(\mathbf{x})-y|
$$

Dada a probabilidade $P(\mathbf{X}, Y)$, o operador ótimo em termos de MAE é dado pela função Booleana assim definida

$$
\psi(\mathbf{x})= \begin{cases}1, & \text { se } P(\mathbf{x}, 1)>0.5 \\ 0, & \text { se } P(\mathbf{x}, 1)<0.5 \\ 1 \text { ou } 0, & \text { para } P(\mathbf{x}, 1)=0.5\end{cases}
$$

para toda realização $\mathbf{x}$ de $\mathbf{X}$.

Na prática, estimativas de $P(\mathbf{X}, Y)$ são empregadas, pois a distribuição real não é conhecida. Mesmo para janelas de tamanho moderado os erros dessa estimativa podem ser grandes. Além disso, janelas grandes criam dificuldades computacionais, pois o algoritmo de aprendizado tem de resolver um problema de otimização maior. A técnica de projeto em dois níveis foi criada para lidar com essas desvantagens das janelas grandes. Em vez de treinar um único operador sobre uma janela grande $W$, esse método divide a mesma área em diversas subjanelas, potencialmente com superposição. Cada uma dessas janelas origina um 
operador, denominado operador de primeiro nível. No segundo nível, as respostas dos primeiros operadores são concatenadas, dando origem a um vetor de atributos. O operador de segundo nível enxerga somente esse vetor e, a partir dele, produz o valor do pixel da imagem de saída. O aprendizado de um operador de dois níveis envolve duas etapas de treinamento distintas, uma para os operadores de primeiro nível e outra para o operador de segundo nível. Na próxima seção será apresentada uma metodologia para automatizar o projeto desse tipo de operador.

\subsection{Um arcabouço para projeto de operadores em dois níveis}

A seleção manual de subjanelas para o projeto de operadores em dois níveis é um processo laborioso. Uma escolha descuidada de janelas pode resultar em redução de desempenho devido à separação de pixels que apresentem interações importantes para a determinação do valor de saída. Ademais, a combinação de operadores somente pode resultar em incremento de desempenho caso estes mostrem algum tipo de diversidade. No caso limite em que todos os operadores representam a mesma função Booleana não é possível haver ganho de precisão nenhum pela combinação dos mesmos. Qualquer procedimento para a escolha automática das janelas deve levar em conta esses dois fatores, a perda de informação e a redundância entre operadores.

Nesta seção será proposta uma metodologia geral para o projeto de operadores em dois níveis, empregando ferramentas de teoria da informação. O primeiro passo dessa metodologia é agrupar os pixels da imagem, usando o Algoritmo 3 , Nós usamos a propriedade de que as linhas da matriz $\mathbf{W}$ devolvida pelo algoritmo podem ser interpretadas como grupos de variáveis binárias. A justificativa para tal procedimento é que esses grupos funcionam como unidades de informação sobre a saída, não devendo ser particionados pela escolha de subjanelas. Tipicamente esses grupos são muito pequenos para serem usados diretamente como janelas. Por isso, no passo seguinte esses grupos são agregados para a formação das subjanelas. Essas subjanelas são empregadas, então, para o treinamento dos operadores de primeiro nível. Nessa etapa o número de operadores treinados costuma ser muito grande. Caso todos eles fossem utilizados o fenômeno do excesso de ajustamento tenderia a ocorrer. Para superar essa dificuldade os operadores são ordenados usando uma medida da informação incremental associada a cada 
operador [Fle04]. Em seguida, um procedimento de seleção de modelos inspirado no princípio do Comprimento Mínimo de Descrição [Grü05, Grü07] (Minimum Description Length - MDL) determina o número de operadores de primeiro nível a ser utilizado no aprendizado do operador de segundo nível. Finalmente, o operador de segundo nível é projetado, usando os operadores de primeiro nível selecionados.

\subsubsection{Construção de subjanelas}

A seleção automática de subjanelas ocorre em um processo em dois passos. Primeiramente, o Algoritmo 3 é empregado sobre um conjunto de pares de imagens (entrada/saída), da mesma maneira que nos experimentos da Seção6.3. Um janela retangular $W$ de tamanho $n$ é adotada, que conterá as subjanelas a serem determinadas. O Algoritmo 3 é aplicado aos dados obtidos dos pares de imagens (entrada/saída), amostrados por meio da janela $W$. A execução do algoritmo gera $n$ diferentes grupos de pixels, correspondentes às linhas da matriz $\mathbf{W}$.

Como exposto ao fim da Seção 6.1, os atributos gerados pelo Algoritmo 3 podem ser interpretados como unidades de informação sobre o rótulo. Relembrando as características da solução produzida pelo algoritmo, o conjunto dos atributos transformados preserva a informação total que os atributos originais continham sobre o rótulo. Cada atributo pós-transformação apresenta pequena redundância em relação aos outros e a quantidade de informação adicional gerada pela observação conjunta de dois atributos também é reduzida. Uma característica da função de paridade é que a inversão de qualquer um dos atributos binários causa inversão também do valor de saída. Logo, podemos dizer que a informação contida em um atributo é característica de todos os elementos que constituem o grupo associado ao atributo. Caso o grupo seja dividido, aquela informação se perderá. $\mathrm{Na}$ aplicação abordada nesta seção, cada grupo constitui um subconjunto dos pixels da janela $W$ (podendo existir intersecção entre esses subconjuntos). Do exposto acima, podemos dizer que cada grupo mostra a existência de uma relação envolvendo todos os pixels do grupo e o pixel de saída. Como o nosso objetivo é criar subjanelas de $W$ e ao mesmo tempo evitar que relações relevantes entre os pixels sejam perdidas nesse processo, justifica-se o uso do Algoritmo 3 para guiar a formação de subjanelas. 
A princípio, os grupos poderiam ser utilizados diretamente como subjanelas de $W$ no procedimento de treinamento em dois níveis. Empiricamente, verificouse que vários dos grupos formados contêm um número pequeno de pixels. Foi testada a utilização de cada um desses grupos diretamente como janelas, mas a escassez de pixels prejudica em demasia o desempenho dos operadores de primeiro nível assim obtidos. Em consequência, o operador de segundo nível não consegue atingir um desempenho satisfatório. Para contornar esse problema, imaginou-se um segundo passo do procedimento de construção de janelas em que grupos são combinados, dando origem às subjanelas dos operadores de primeiro nível.

A agregação dos grupos procede do seguinte modo: os grupos são ordenados de maneira decrescente de acordo com a informação $I\left(Y ; Z_{i}\right)$ fornecida pelo atributo $Z_{i}$ associado a cada grupo de variáveis. Deve-se lembrar que

$$
Z_{i}=\mathbf{w}_{i} \mathbf{X}
$$

em que $\mathbf{w}_{i}$ é uma linha da matriz $\mathbf{W}$ gerada pelo Algoritmo 3 . Como há uma correspondência biunívoca entre grupos e linhas da matriz $\mathbf{W}$, temos $n$ grupos no total. Sejam esses grupos denotados por

$$
G_{1}, G_{2}, \ldots, G_{n}
$$

depois do ordenamento. O procedimento de agregação concebido gera um número de janelas igual ao número de grupos. Denote-se por

$$
W_{1}, W_{2}, \ldots, W_{n}
$$

as subjanelas a serem geradas pelo processo de agregação dos grupos. A continuação do processo de agregação é descrita pelo Algoritmo 4. O algoritmo recebe uma parâmetro TAMANHO_MAXIMO que limita a quantidade de pixels que uma subjanela pode ter após a agregação. Dois fatores orientam a seleção desse parâmetro. Computacionalmente, uma janela muito grande aumenta consideravelmente o tempo de execução. O número excessivo de pixels poderia gerar também o fenômeno do excesso de ajustamento. No início do Algoritmo 4 cada janela corresponde a um dos $n$ grupos. O algoritmo passa a iterar sobre os grupos e 
as janelas, tentando a cada passo unir o grupo em questão com uma janela diferente, verificando se a operação de união não fez a janela ultrapassar o limite TAMANHO_MAXIMO. Com esse procedimento, os grupos que são mais informativos sobre a saída têm maiores chances de serem incorporados às janelas, ao mesmo tempo que nenhum grupo é desprezado. Após esse processo, podem existir janelas duplicadas, que são removidas em um passo de filtragem. O processo de agregação é ilustrado na Figura 7.1. Neste exemplo, o grupo $G_{1}$ é adicionado às janelas $W_{2}$ e $W_{3}$. Depois disso, nenhum grupo pode ser adicionado a essas janelas sem violar a restrição do tamanho máximo. Ao fim do processo, as janelas $W_{1} \mathrm{e}$ $W_{2}$ são idênticas, logo uma delas deve ser removida.

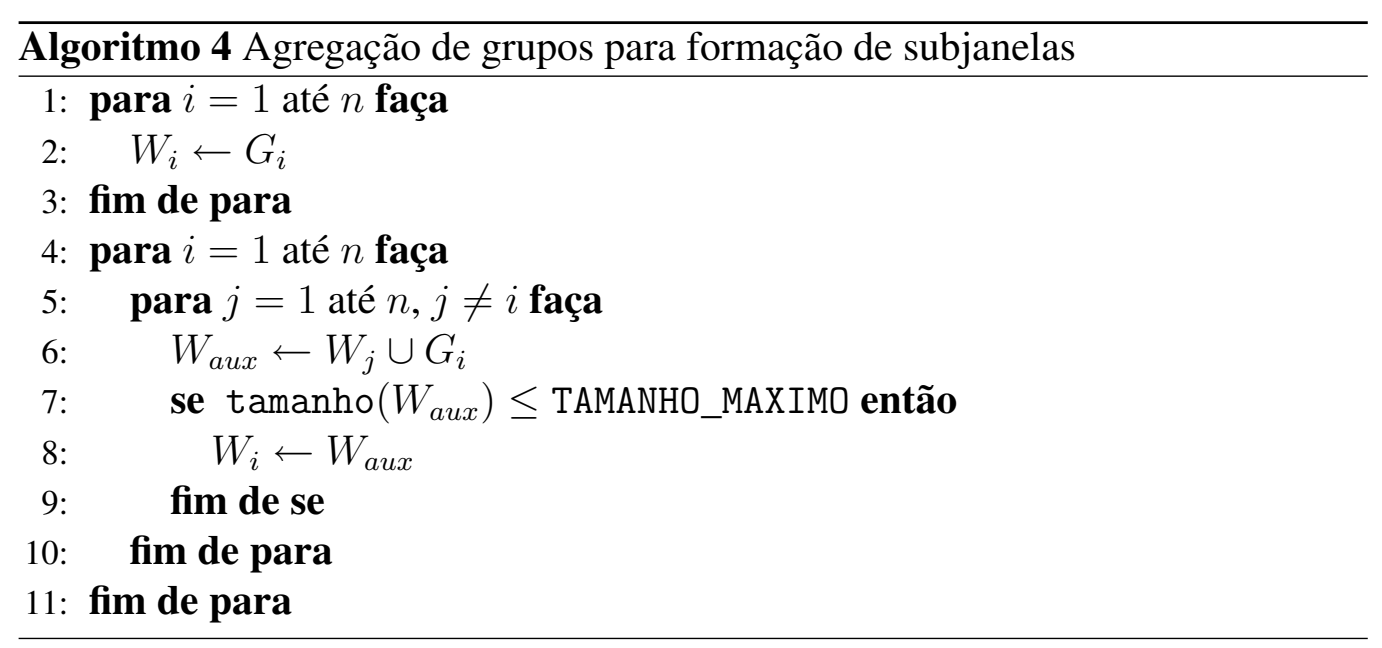

\subsubsection{Seleção de Operadores}

O procedimento descrito na Seção 7.3.1 usa a interação entre pixels para gerar subjanelas que dividem a área observada da imagem e ao mesmo tempo preservam a informação sobre a imagem de saída. No entanto, o número de operadores de primeiro nível obtidos ao se utilizar todas essas janelas pode ser excessivo e muitos dos operadores podem ser redundantes. Para selecionar os operadores empregados pelo operador de segundo nível pode-se utilizar técnicas de seleção de atributos e comparação de modelos. Nesse contexto, as saídas dos operadores de primeiro nível são tratadas como um novo conjunto de atributos e o rótulo desejado é ainda o pixel correspondente da imagem de saída.

Sob o ponto de vista da teoria da informação, um procedimento de seleção 


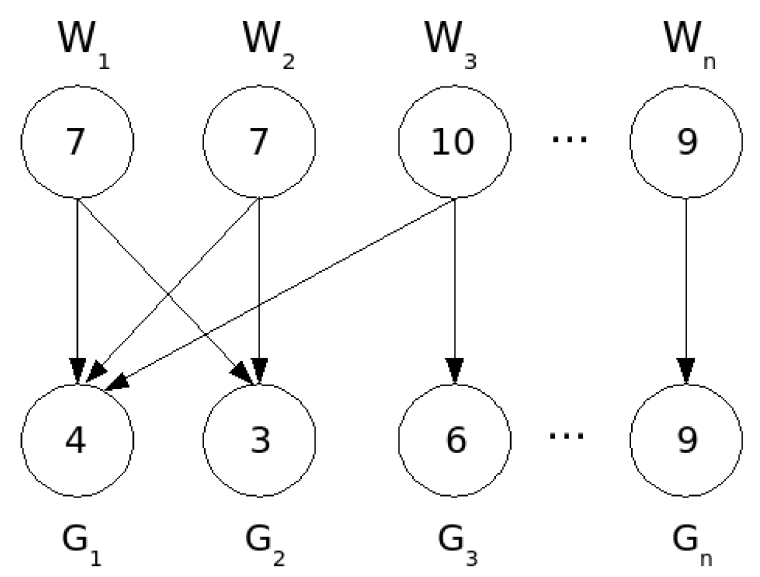

Figura 7.1: Ilustração da agregação de grupos. Nesse caso, TAMANHO_MAXIMO é igual a 11. O número contido em cada círculo indica o tamanho de cada grupo ou janela. Cada janela contém ao menos o grupo de mesmo índice. As setas indicam os grupos agregados para a formação da janela.

de atributos deveria maximizar a informação que o conjunto selecionado fornece sobre o rótulo de saída. No entanto, o cálculo da informação mútua entre rótulo e atributos demandaria a estimativa de distribuições de probabilidade multidimensionais, causando problemas com relação à precisão estatística. Em vez de maximizar a informação mútua diretamente, optou-se pelo critério de Maximização da Informação Mútua Condicional (Conditional Mutual Information Maximization - CMIM) [Fle04] que visa minimizar a redundância entre atributos. O procedimento CMIM é um algoritmo sequencial de seleção de atributos que ordena os mesmos iterativamente de acordo como seu poder de predição em relação à saída mas descontando a redundância de cada atributo com relação àqueles previamente escolhidos. Sendo $Y$ um rótulo e $X_{1}, X_{2}, \ldots, X_{n}$ os atributos de predição, o algoritmo determina um conjunto de índices

$$
\nu(1), \nu(2), \ldots, \nu(m)
$$

em que $m<n$, de modo que o subconjunto selecionado

$$
X_{\nu(1)}, X_{\nu(2)}, \ldots, X_{\nu(m)}
$$


seja ao mesmo tempo informativo sobre $Y$ e pouco redundante entre si. O algoritmo é descrito pelo seguinte esquema iterativo:

$$
\nu(1)=\arg \max _{i} I\left(Y ; X_{i}\right)
$$

E para $k>1$ :

$$
\nu(k)=\arg \max _{i}\left\{\min _{j<k} I\left(Y ; X_{i}, X_{\nu(j)}\right)-I\left(Y ; X_{\nu(j)}\right)\right\}
$$

O primeiro atributo selecionado pelo algoritmo CMIM é o mais informativo, isoladamente. Nos passos seguintes, para cada um dos atributos restantes, calcula-se uma função de qualidade igual a

$$
Q\left(X_{i}, k\right)=\min _{j<k} I\left(Y ; X_{i}, X_{\nu(j)}\right)-I\left(Y ; X_{\nu(j)}\right)
$$

em que $X_{i}$ é um atributo candidato para seleção e $X_{\nu(j)}$ é algum dos atributos previamente escolhidos. O termo

$$
I\left(Y ; X_{i}, X_{\nu(j)}\right)-I\left(Y ; X_{\nu(j)}\right)
$$

expressa a informação adicional que $X_{i}$ fornece sobre o rótulo com relação a $X_{\nu(j)}$. Ao percorrermos todo o conjunto de atributos pré-selecionados, calculando o valor mínimo de (7.5), estamos estimando de maneira conservadora a informação adicional que $X_{i}$ pode fornecer sobre o rótulo se adicionado ao conjunto de atributos. Para calcular essa informação de maneira exata seria necessário estimar

$$
P\left(Y, X_{i}, X_{\nu(1)}, X_{\nu(2)}, \ldots, X_{\nu(k-1)}\right)
$$

incorrendo-se nas dificuldades de estimação multidimensional citadas anteriormente. Em resumo, para um atributo receber uma medida de qualidade alta pelo procedimento CMIM é preciso que esse atributo seja informativo sobre o rótulo e não redundante com nenhum atributo selecionado previamente. O cálculo da função de qualidade $Q\left(X_{i}, k\right)$ é bastante eficiente, pois exige a estimativa de distribuições de probabilidade de no máximo 3 variáveis e também porque certas otimiza- 
ções dispensam a atualização do cálculo da função em algumas situações [Fle04].

O critério CMIM provê um meio de ordenar os operadores de primeiro nível mas deixa em aberto a questão de quantos operadores devem ser utilizados. Nós abordamos essa questão com um método que adapta idéias do princípio do Comprimento Mínimo de Descrição (Minimum Description Length - MDL) [Grü07] para seleção de modelos. Nesse contexto, a palavra modelo refere-se a uma categoria de classificadores, onde cada classificador é especificado por um conjunto de parâmetros $\theta$. No problema tratado aqui, denotaremos por $M_{k}$ a categoria dos operadores de segundo nível que empregam $k$ operadores de primeiro nível. Uma vez que esses operadores equivalem a funções Booleanas de $k$-variáveis, o conjunto de parâmetros $\theta_{k}$ equivale aos parâmetros necessários para especificar a tabela da verdade de uma função Booleana de $k$ variáveis. A maneira mais imediata de especificar uma função Booleana é declarando o valor binário de saída para cada uma das $2^{k}$ entradas possíveis. Nesse caso o conjunto $\theta_{k}$ é formado por esses $2^{k}$ valores binários. Entre as categorias definidas acima, é válida a relação:

$$
M_{2} \subset M_{3} \subset M_{4} \subset \ldots \subset M_{k}
$$

pois a classe de funções Booleanas de até $k$ variáveis engloba a classe de funções Booleanas de até $m$ variáveis, para $k>m$.

Dado um conjunto de realizações

$$
\left(\mathbf{x}_{1}, y_{1}\right),\left(\mathbf{x}_{2}, y_{2}\right), \ldots,\left(\mathbf{x}_{L}, y_{L}\right)
$$

o princípio MDL trata a seleção de modelos como o problema de encontrar uma representação compacta para o vetor de rótulos

$$
\mathbf{y}=\left(y_{1}, y_{2}, \ldots, y_{L}\right)
$$

considerando que a sequência de atributos

$$
\mathbf{x}_{1}, \mathbf{x}_{2}, \ldots, \mathbf{x}_{L}
$$

é conhecida. Seja $M$ um modelo, associado ao conjunto de parâmetros $\theta$ e utili- 
zado para codificar um vetor de rótulos y. De acordo com a teoria da informação, o menor número de bits necessários para codificar $\theta$ é determinado por [Grü07] [HTF01, Ch. 7]

$$
\operatorname{comprimento}(\theta)=-\log P(\theta \mid M)
$$

O termo expresso na Equação (7.6) impõe uma penalidade a modelos muito complexos, que precisam de mais parâmetros para serem codificados. Se o conjunto de treinamento for gerado de maneira independente e identicamente distribuída (i.i.d), o número de bits necessários para codificar os rótulos usando o modelo $M$ será [Grü07][HTF01, Ch.7]:

$$
\text { comprimento rótulos }=-\sum_{i=1}^{L} \log P\left(y_{i} \mid \theta, M, \mathbf{x}_{i}\right)
$$

O termo expresso na Equação (7.7) impõe uma penalidade a modelos que não conseguem se ajustar aos dados. Para enxergar isso, considere o par $\left(\mathbf{x}_{i}, y_{i}\right)$ pertencente ao conjunto de treinamento. Caso o modelo não seja capaz de se ajustar ao conjunto de treinamento, a probabilidade atribuída ao valor $y_{i}$ quando a entrada é igual a $\mathbf{x}_{i}$ será pequena e a penalidade

$$
-\log P\left(y_{i} \mid \theta, M, \mathbf{x}_{i}\right)
$$

tende a crescer. O contrário ocorre ajustamento aos dados, o modelo tende a atribuir probabilidade alta para as combinações $\left(\mathbf{x}_{i}, y_{i}\right)$ encontradas no conjunto de treinamento e a penalidade dada por (7.7) será pequena.

A função de qualidade MDL é dada pelo comprimento total da representação (modelo + rótulos codificados pelo modelo), calculada por meio de

$$
\text { comprimento total }=-\sum_{i=1}^{L} \log P\left(y_{i} \mid \theta, M, \mathbf{x}_{i}\right)-\log P(\theta \mid M)
$$

O princípio MDL recomenda a adoção do modelo com menor comprimento. Modelos excessivamente complexos conseguem codificar os rótulos de maneira compacta, pois são mais capazes de se ajustar aos dados. Ao mesmo tempo, esses modelos possuem mais parâmetros/graus de liberdade a serem especificados, au- 
mentando o comprimento final da descrição. Os modelos excessivamente simples, pelo contrário, podem ser descritos por um número menor de parâmetros, sendo codificados eficientemente, mas sua incapacidade de se ajustar aos dados torna necessária uma descrição de maior comprimento para os rótulos. O princípio MDL privilegia modelos razoavelmente complexos, possibilitando o ajuste aos dados e a codificação eficiente dos rótulos, ao mesmo tempo conservando uma descrição simples para o próprio modelo.

No nosso caso, se $k$ operadores de primeiro nível são utilizados para treinar o operador de segundo nível, o número de configurações de entrada distintas que podem ser enxergadas pelo operador de segundo nível é igual a $2^{k}$. O modelo para o operador de segundo nível consistirá então de uma tabela com $2^{k}$ linhas, uma para cada possível configuração de entrada. Cada linha associa uma configuração de entrada a um parâmetro binário, que determina se a saída do operador de segundo nível deve adotar valor 0 ou 1 para aquela configuração. Logo, nosso modelo requer $2^{k}$ bits para ser especificado. Outro modo de derivar esse resultado parte do fato que a classe de funções Booleanas de $k$ variáveis contém $2^{2^{k}}$ membros. Supondo todas as funções equiprováveis a priori, a probabilidade de qualquer função $\psi$ é dada por

$$
P(\psi)=1 / 2^{2^{k}}
$$

Usando esse resultado na Equação (7.6) temos

$$
\operatorname{comprimento}(\theta)=\log _{2} 2^{2^{k}}=2^{k} \text { bits }
$$

Sendo $\Psi(\mathbf{x})$ a resposta do operador de dois níveis a uma entrada $\mathbf{x}$, a função de comprimento de rótulos consistente com a codificação de modelo adotada é dada por [Vap98, cap. 6]

$$
\text { comprimento rótulos }=-\sum_{i=1}^{L} \log P\left(y_{i} \mid \Psi\left(\mathbf{x}_{i}\right)\right)
$$

No entanto, testes realizados com a função 7.10 mostraram uma tendência final a subestimar o número de operadores. Foi verificado que a função (7.10) tinha 
pouco peso frente ao termo de complexidade do modelo (7.9). Este prevalecia, indicando um número menor de operadores do que seria adequado.

Após alguma experimentação, adotou-se a seguinte função, que pode ser interpretada como o comprimento do código necessário para codificar os rótulos a partir das respostas dos operadores de primeiro nível:

$$
\text { comprimento rótulos }=-\sum_{i=1}^{L} \log P\left(y_{i} \mid \psi_{1}\left(\mathbf{x}_{i}\right), \ldots, \psi_{k}\left(\mathbf{x}_{i}\right)\right) \text {, }
$$

onde $\psi_{k}(\mathbf{x})$ denota a resposta do $k$-ésimo operador de primeiro nível.

Assim, a função de qualidade usada para avaliar o modelo foi definida como:

$$
\text { comprimento total }=-\sum_{i=1}^{L} \log P\left(y_{i} \mid \psi_{1}\left(\mathbf{x}_{i}\right), \ldots, \psi_{k}\left(\mathbf{x}_{i}\right)\right)+2^{k}
$$

Para avaliação dos modelos, a função (7.12) é calculada para valores crescentes de $k$. O número de operadores de primeiro nível recomendado pelo método proposto corresponde ao valor de $k$ para o qual a função da Equação (7.12) obtém seu valor mais baixo, isto é, o que propicia menor comprimento de descrição. A Equação (7.12) não corresponde exatamente ao princípio MDL, pois a saída do modelo (i.e. a saída do operador de segundo nível) não é usada para calcular a probabilidade dos rótulos, usando-se em seu lugar a probabilidade conjunta dos operadores de primeiro nível.

\subsection{Descrição dos experimentos}

Para testar a metodologia proposta de projeto de operadores em dois níveis, um esquema experimental foi criado e aplicado em diferentes tarefas de processamento de imagens binárias. Cada tarefa é associada a um conjunto de pares de imagens (imagem de entrada, imagem desejada). Cada um desses conjuntos foi dividido em dois subconjuntos disjuntos, um de treinamento e outro de teste, visando que os operadores gerados fossem avaliados em um conjunto de imagens não empregadas durante o treinamento.

Para cada tarefa, primeiro o Algoritmo 3 foi usado para agrupar os pixels e os grupos formados foram agregados em janelas de acordo como o procedimento 
descrito na Seção 7.3.1. Na aplicação do Algoritmo 3, o número de iterações MAX_ITER foi escolhido de modo que cada um dos pares de índices $(i, j)$ possíveis $(i \neq j)$ fosse empregado 5 vezes. Em todos os experimentos, o número máximo de pixels em cada janela foi limitado em 36 durante o processo de agregação. Essa escolha foi feita empiricamente para limitar o tempo de treinamento dos operadores de primeiro nível mas também para evitar o excesso de ajustamento. Cada subjanela foi utilizada então para treinar um operador de primeiro nível. Esse operadores foram então aplicados sobre as imagens de treinamento, resultando em um conjunto de imagens intermediárias. As imagens intermediárias são tratadas como novos atributos e passadas para o procedimento CMIM, que ordena os operadores. O treinamento dos operadores de primeiro nível e de segundo nível utilizou o algoritmo Incremental Splitting of Intervals (ISI) de minimização de funções Booleanas [HBD02], empregado com sucesso no projeto de operadores binários [HBD02, Hir09], tal como implementado na biblioteca PAC [BTHH00].

Em seguida, uma série de operadores de segundo nível é treinada, cada um empregando um número crescente de operadores de primeiro nível, seguindo a ordem determinada pelo critério CMIM. Em outras palavras, o primeiro operador treinado nesta etapa emprega os dois melhores operadores da etapa anterior como ordenados pelo o critério CMIM. Os operadores treinados em seguida adicionam um operador de primeiro nível ao conjunto usado, de acordo com a ordem imposta pelo critério CMIM. Os operadores de segundo nível assim obtidos foram avaliados sobre o conjunto de imagens de teste. Para cada um dos operadores de segundo nível foi também calculado o índice de qualidade de acordo com o procedimento de comparação de modelos descrito na Seção 7.3.2. Nas próximas seções, serão comparados os operadores de segundo nível escolhidos de acordo com o procedimento de seleção de modelos e aqueles empiricamente determinados como os de melhor desempenho no conjunto de teste.

\subsection{Segmentação de texto em imagens}

A identificação de regiões preenchidas por texto é uma tarefa importante no processamento e análise de documentos. Neste experimento, a tarefa consistiu em localizar as regiões de texto em imagens digitalizadas de páginas de revistas, 
contendo uma mistura de texto e ilustrações. O conjunto de treinamento consistia de 10 pares (imagem de entrada, imagem desejada). Cinco pares foram atribuídos aleatoriamente ao conjunto de treinamento e o cinco restantes formaram o conjunto de teste. Para o treinamento dos operadores em dois níveis o conjunto de treinamento foi dividido novamente, 3 pares de imagens foram usados para determinar as janelas e para o treinamento dos operadores de primeiro nível. Os outros 2 pares foram empregados para treinar os operadores de segundo nível. Uma janela quadrada $11 \times 11$ foi utilizada como a janela externa que contém as subjanelas. Algumas das janelas resultantes são mostradas na Figura 7.2 .
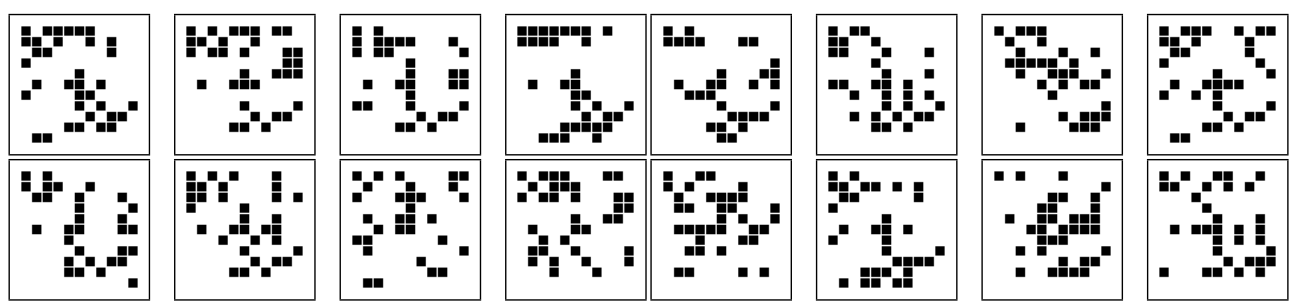

Figura 7.2: Algumas janelas dos operadores de primeiro nível obtidas para a tarefa de segmentação de texto.

A Figura 7.3 mostra a evolução do erro (medido pelo MAE, ver equação 7.1) cometido pelo operador de segundo nível conforme são acrescentados novos operadores. A mesma figura ilustra a função de qualidade do procedimento de comparação de modelos, dada pela fórmula (7.12). Como se depreende da figura, o mínimo da função de seleção de modelos não coincide com o mínimo do erro de teste. A Tabela 7.1 compara o operador de dois níveis com melhor desempenho de teste com o operador recomendado pelo procedimento de seleção de modelos. Embora o operador recomendado seja um pouco mais complexo, usando 12 operadores no primeiro nível contra 9 do melhor operador empírico, a diferença de desempenho entre os dois, medida pelo MAE, é quase nula. A Figura 7.4 mostra o resultado obtido para o melhor operador encontrado empiricamente, para uma imagem do conjunto de teste.

\subsection{Reconhecimento de Texturas}

Neste experimento, o projeto de operadores em dois níveis foi utilizado em uma tarefa de reconhecimento de textura em imagens digitalizadas de mapas. $\mathrm{O}$ 

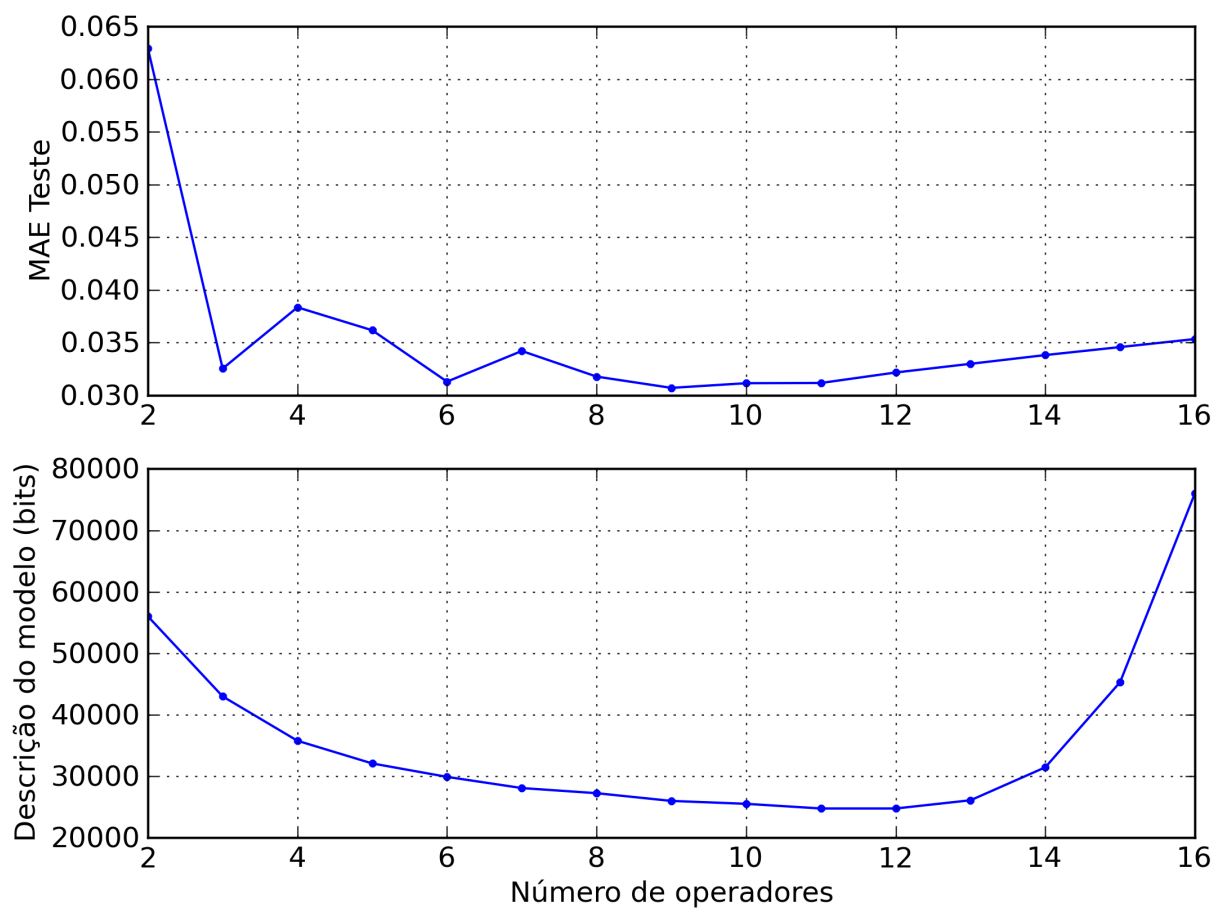

Figura 7.3: Tarefa de reconhecimento de texto. Acima: MAE medido sobre as imagens de teste como função do número de operadores de primeiro nível usados. Em baixo: função de qualidade usada pelo procedimento de comparação de modelos.

\begin{tabular}{|c|c|c|}
\cline { 2 - 3 } \multicolumn{1}{c|}{} & Número de operadores de $1^{\circ}$ nível & MAE \\
\hline Melhor operador, 2 níveis & 9 & 0.031 \\
\hline Operador recomendado, 2 níveis & 12 & 0.032 \\
\hline
\end{tabular}

Tabela 7.1: Tarefa de reconhecimento de texto. Comparação entre o melhor operador de dois níveis, encontrado empiricamente e o operador recomendado pelo procedimento de comparação de modelos.

objetivo é obter uma imagem de saída que contenha apenas as regiões com um determinado padrão de textura. Foram utilizados dois pares de imagens para escolha das janelas e treinamento dos operadores de primeiro nível. Um único par foi usado para treinar os operadores de segundo nível e dois outros pares foram empregados para teste. A amostragem foi realizada com uma janela quadrada com 9 pixels de lado. Algumas das janelas obtidas são mostradas na Figura 7.5.

A Figura 7.6 mostra a evolução do MAE do operador de segundo nível em 


\begin{tabular}{|c|c|c|}
\cline { 2 - 3 } \multicolumn{1}{c|}{} & Número de operadores de $1^{\circ}$ nível & MAE \\
\hline Operador de um nível & - & 0.07 \\
\hline Melhor operador, 2 níveis & 8 & 0.060 \\
\hline Operador recomendado, 2 níveis & 11 & 0.061 \\
\hline
\end{tabular}

Tabela 7.2: Tarefa de reconhecimento de textura. MAE de teste para um operador simples, em um nível, para o melhor operador em dois níveis encontrado e para o operador em dois níveis recomendado pelo procedimento de comparação de modelos.

função do número de operadores de primeiro nível, junto com a função de comparação de modelos. Novamente, o operador selecionado pelo procedimento de comparação de modelos utiliza mais operadores que o operador de melhor desempenho de teste. A Tabela 7.2 compara esses dois operadores e inclui também o MAE para um operador simples, de um nível, que usa a mesma janela $9 \times 9$. Ambos operadores de 2 níveis apresentam melhor desempenho que o operador simples, mostrando que a divisão em subjanelas levou a um aproveitamento melhor da mesma informação sobre a imagem. A Figura 7.7 mostra o resultado obtido para o melhor operador encontrado empiricamente, para uma imagem do conjunto de teste.

\subsection{Identificação de caracteres}

Neste experimento, o objetivo é identificar um determinado caractere (neste caso, a letra "s") em imagens de páginas digitalizadas a partir de um livro. O conjunto de treinamento consistiu de 10 pares de imagens (6 pares para geração de janelas e treinamento dos operadores de primeiro nível e 4 pares para o treinamento dos operadores de segundo nível). Outros 10 pares de imagens foram usados para testar os operadores de segundo nível. A amostragem usou uma janela de 7 pixels de largura por 9 de altura. Algumas das subjanelas obtidas para o projeto dos operadores de primeiro nível são mostradas na Figura 7.8.

O comportamento do MAE e da função de comparação de modelos são mostrados n Figura 7.9. O desempenho do melhor operador e do operador recomendado pelo procedimento de seleção de modelos são comparados na Tabela 7.3 compara esses dois operadores e inclui também o MAE para um operador simples, de um nível, empregando a mesma janela $7 \times 9$. Novamente, os operadores de dois níveis superam o operador simples. A diferença entre o melhor opera- 


\begin{tabular}{|c|c|c|}
\cline { 2 - 3 } \multicolumn{1}{c|}{} & Número de operadores de $1^{\circ}$ nível & MAE \\
\hline Operador de um nível & - & 0.009 \\
\hline Melhor operador, dois níveis & 7 & 0.0073 \\
\hline Operador recomendado, dois níveis & 12 & 0.0079 \\
\hline
\end{tabular}

Tabela 7.3: Tarefa de reconhecimento de caractere. Comparação entre o melhor operador de dois níveis, operador recomendado pelo procedimento de seleção de modelos e um operador de um nível que usa uma janela $7 \times 9$.

dor, determinado empiricamente, e o operador recomendado pelo procedimento de comparação de modelos não foi significativa. Para uma imagem com 10.000 pixels, o operador recomendado comete, em média, 6 erros a mais que o melhor operador. A Figura 7.10 mostra o resultado obtido para o melhor operador encontrado empiricamente, para uma imagem do conjunto de teste.

\subsection{Comparação com janelas selecionadas manualmente}

Nesta seção será feita a comparação de resultados obtidos com a metodologia proposta e resultados publicados [Hir09] na literatura, obtidos com operadores de dois níveis em que as janelas dos operadores de primeiro nível foram selecionadas manualmente. Os experimentos mostrados nesta seção reproduzem fielmente as condições dos experimentos realizados por Nina Hirata [Hir09], possibilitando a comparação direta dos mesmos. A Tabela 7.4 resume a comparação entre os resultados obtidos com os dois métodos de seleção. Para o método de seleção automática, os resultados mostrados na Tabela 7.4 correspondem ao melhor operador determinado sobre o conjunto de teste. Deve-se notar que os resultados obtidos com seleção manual são consequência de um longo processo de tentativa e erro com diversos formatos de janelas, portanto é razoável supor que eles estejam próximos do melhor resultado que pode ser obtido com operadores de dois níveis para cada uma das tarefas examinadas. Nota-se que os dois procedimentos de seleção de janela coincidem em desempenho para a tarefa de segmentação de texto e o método de seleção manual somente tem uma vantagem mais pronunciada na tarefa de reconhecimento de textura. A comparação visual entre as janelas usadas por Nina Hirata [Hir09] e as obtidas nesta seção mostram que estas últimas apresentam menor diversidade espacial, existindo maior sobreposição entre as janelas selecionadas automaticamente. Isso mostra que o procedimento proposto para a 


\begin{tabular}{|c|c|c|c|}
\cline { 2 - 4 } \multicolumn{1}{c|}{} & \multicolumn{3}{c|}{ MAE } \\
\cline { 2 - 4 } \multicolumn{1}{c|}{} & Texto & Textura & Caractere \\
\hline Seleção manual* & 0.031 & 0.04 & 0.006 \\
\hline Seleção automática & 0.031 & 0.060 & 0.0073 \\
\hline
\end{tabular}

Tabela 7.4: Comparação entre operadores obtidos com seleção manual de janelas e os obtidos pelo método proposto nesta seção, para as três tarefas previamente descritas. * Dados provenientes de [Hir09].

agregação de grupos e formação de janelas (Algoritmo 4) pode ser aperfeiçoado pela inclusão de um mecanismo que estimule a diversidade entre janelas. 


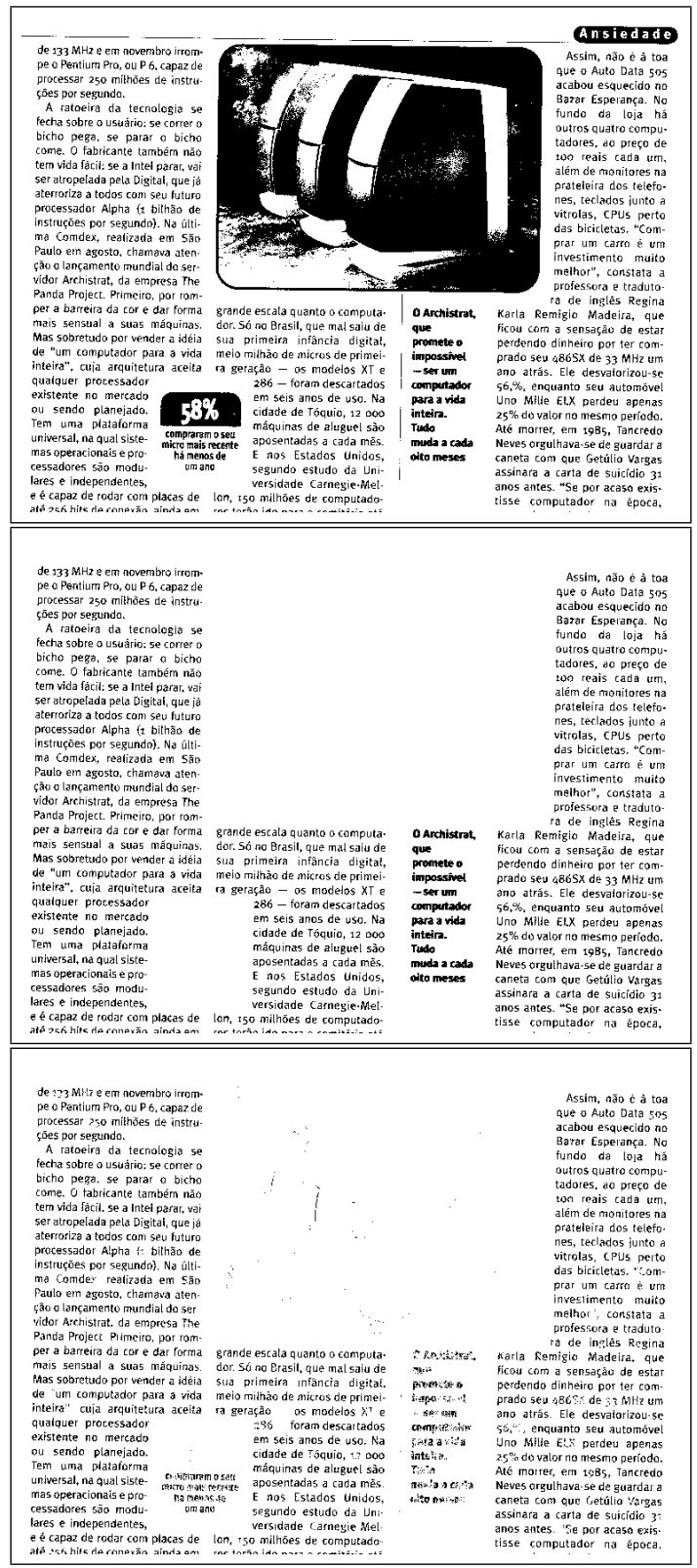

Figura 7.4: Imagens de segmentação de regiões de texto. No topo, imagem de entrada do conjunto de teste. Centro: imagem desejada. Embaixo: imagem obtida com o melhor operador de dois níveis. 


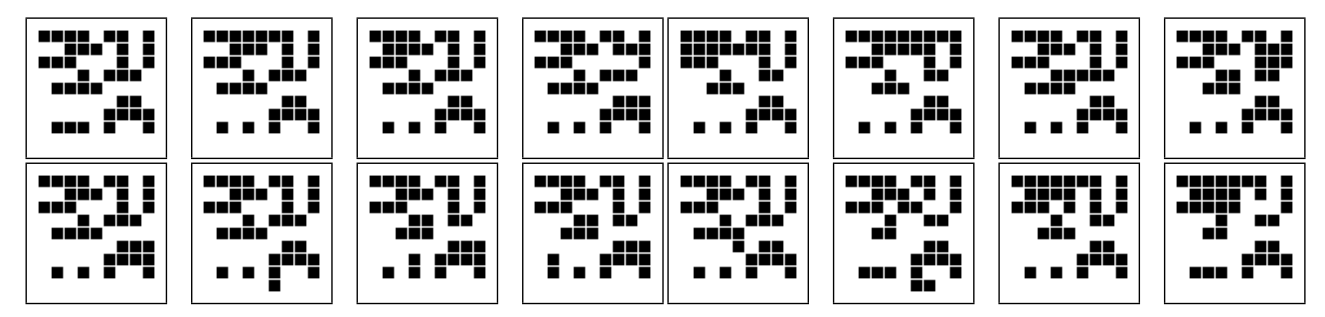

Figura 7.5: Algumas janelas dos operadores de primeiro nível obtidas para a tarefa de reconhecimento de textura.
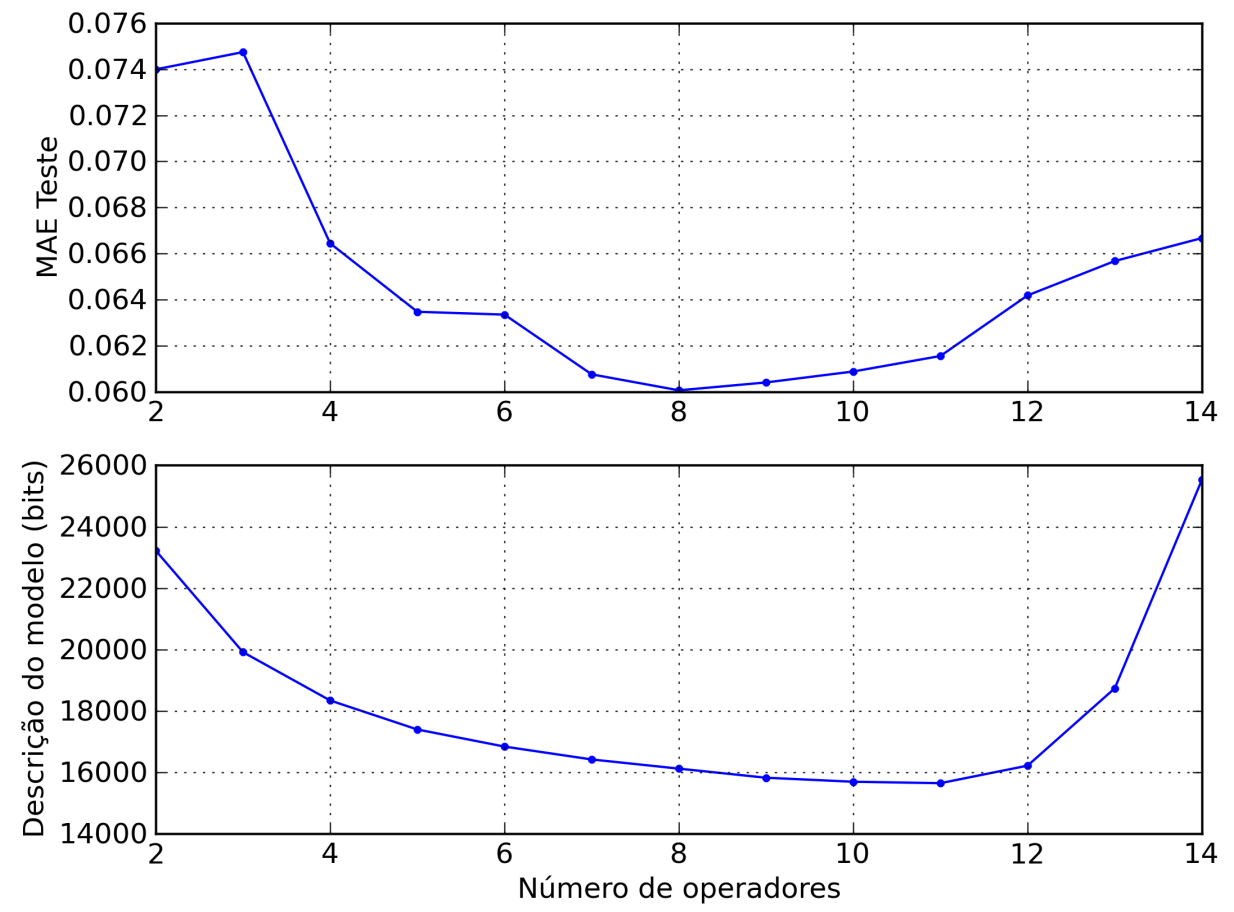

Figura 7.6: Tarefa de reconhecimento de textura. Acima: MAE medido sobre as imagens de teste como função do número de operadores de primeiro nível usados. Em baixo: função de qualidade usada pelo procedimento de comparação de modelos. 


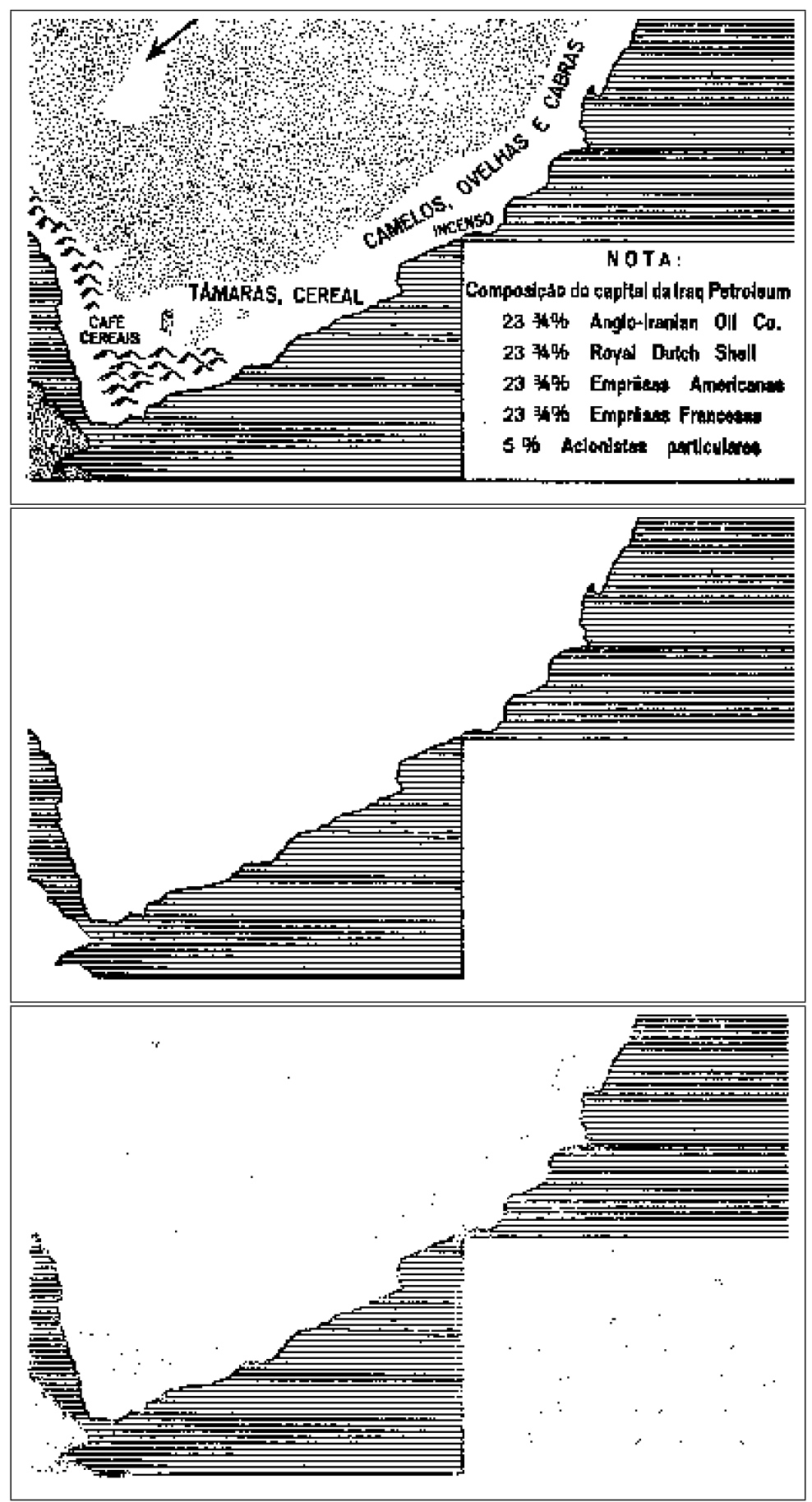

Figura 7.7: Imagens para reconhecimento de textura. Imagem de entrada (topo), imagem desejada (centro) e imagem obtida com o melhor operador de dois níveis (embaixo). 


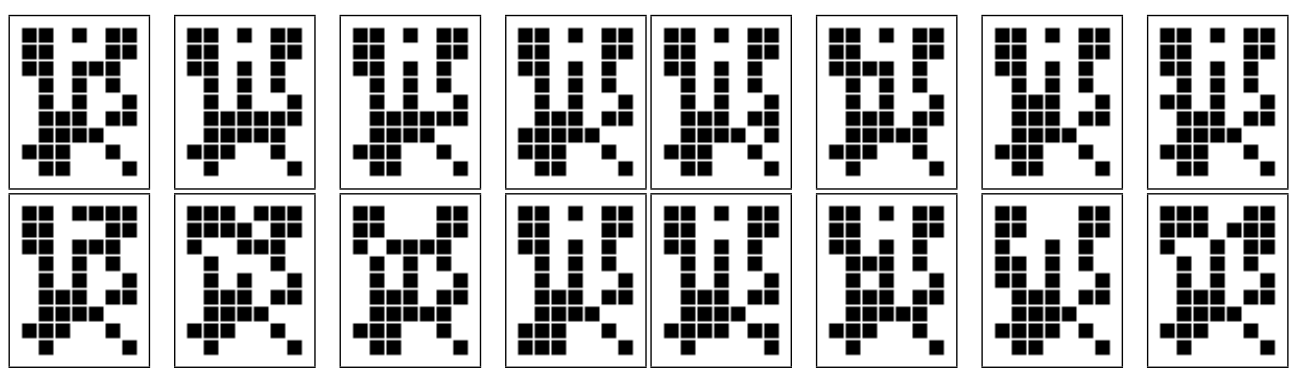

Figura 7.8: Algumas janelas dos operadores de primeiro nível obtidas para a tarefa de reconhecimento de caractere.
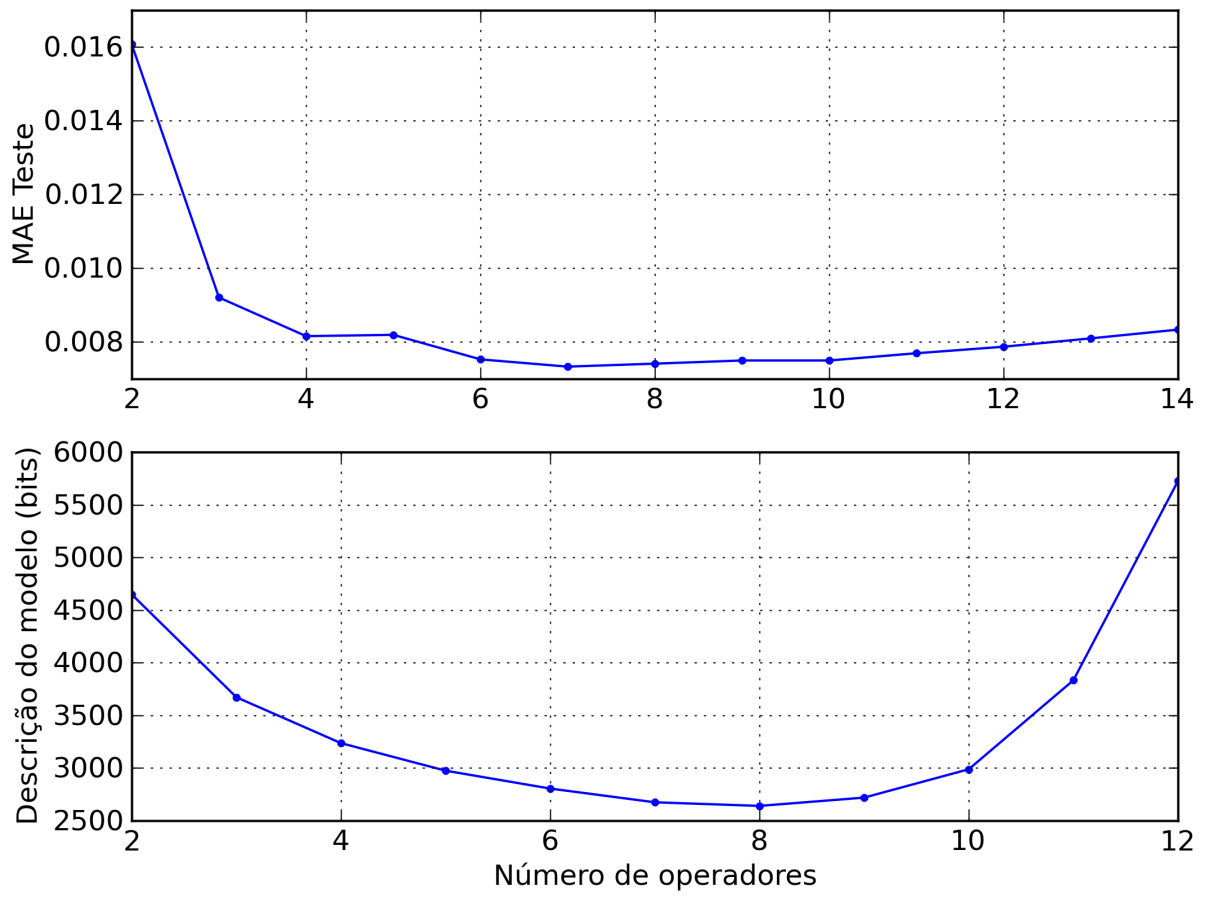

Figura 7.9: Tarefa de reconhecimento de caractere. Acima: MAE medido sobre as imagens de teste como função do número de operadores de primeiro nível usados. Em baixo: função de qualidade usada pelo procedimento de comparação de modelos. 


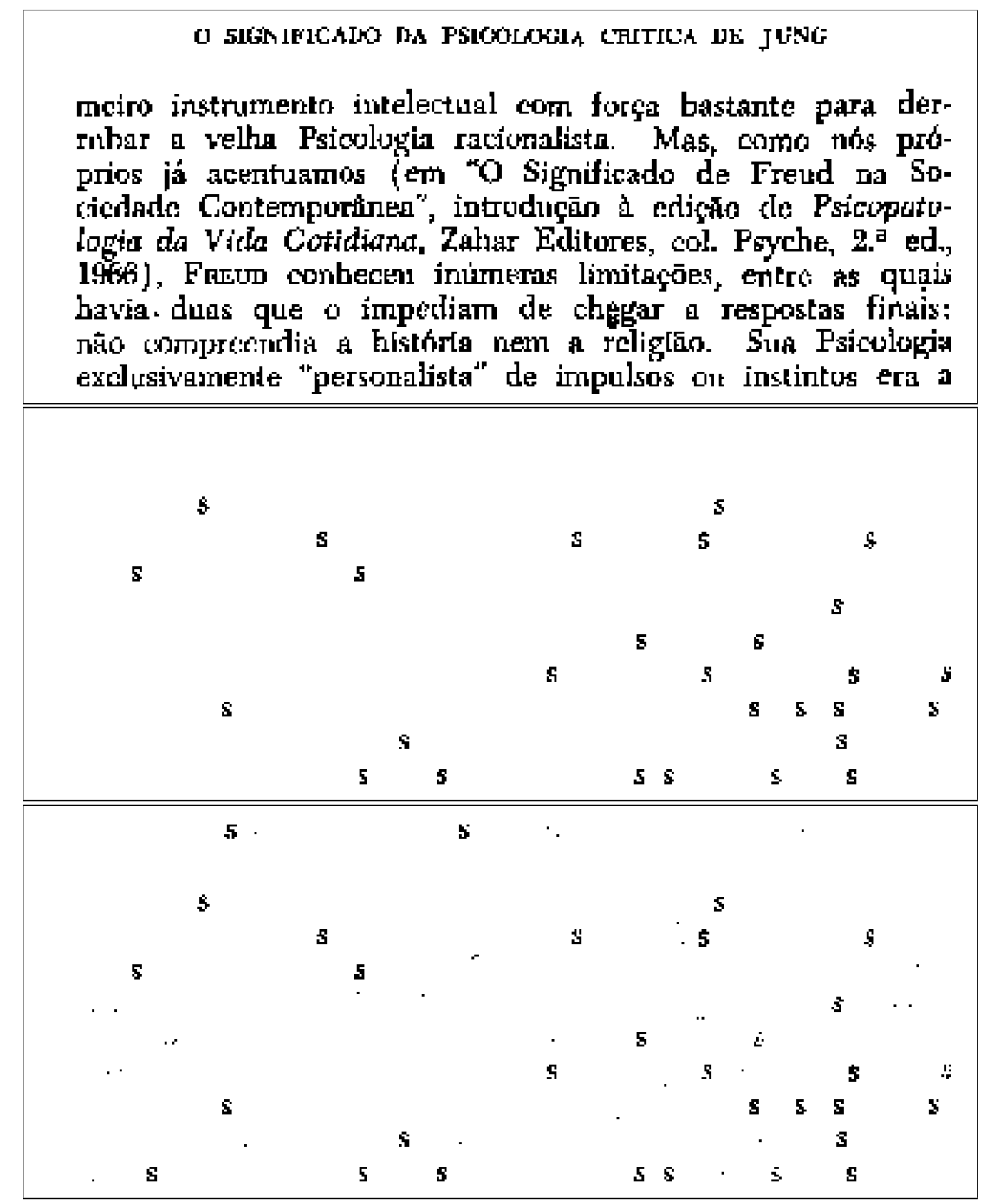

Figura 7.10: Imagens para identificação de caractere. Imagem de entrada (topo), imagem desejada (centro) e imagem obtida com o melhor operador de dois níveis (embaixo). 


\section{Capítulo 8}

\section{Conclusões}

Neste texto foi apresentado um novo método para a transformação de atributos binários, baseado na construção iterativa de uma matriz de paridade. Foi demonstrado que esse tipo de transformação é inversível e conserva a entropia conjunta dos atributos, ao mesmo tempo permitindo manipular quantidades relacionadas às distribuições marginais dos mesmos. Essa propriedade foi utilizada para definir três métodos que minimizam: i) a multi-informação dos atributos (ICA binária, Capítulo 4), ii) a multi-informação condicional dos atributos, dado um rótulo (Capítulo 5) e iii) a informação de interação média entre atributos (Capítulo 6). Embora o uso de matrizes de paridade seja comum na construção de códigos lineares [BR98, Mac03], durante a execução deste trabalho não foram encontradas referências anteriores que empregassem tal tipo de matriz para a construção de atributos no contexto de reconhecimento de padrões.

O método de ICA binária e o método de redução de multi-informação foram estudados quanto a suas características de convergência e complexidade computacional. Foi mostrado que apesar de atuarem para reduzir, respectivamente, a multiinformação e a multi-informação condicional, nenhum dos métodos pode garantir uma solução ótima. Para o algoritmo de ICA, a qualidade da solução tende a aumentar quando o algoritmo trabalha com dados binários. O algoritmo de ICA binária pode ser usado para induzir esparsidade dos atributos em situações em que essa característica é desejável, por exemplo, em memórias associativas [Ama89]. Um possível uso do algoritmo de minimização da multi-informação condicional é no aprendizado de modelo probabilísticos, pois a eliminação de dependências condicionais colabora para a obtenção de modelos simplificados. 
O método de construção de atributos por redução das interações é heurístico, pois não pode ser associado a uma função objetivo a ser otimizada. De maneira empírica, foi mostrado que esse método resulta na diminuição da magnitude média das interações entre atributos. O efeito dessa diminuição é reduzir a redundância entre atributos ao mesmo tempo transferindo informação contida em interações entre pares de atributos para atributos individuais. Tais atributos, após a transformação, podem ser entendidos como blocos de informação sobre o rótulo, em problemas de classificação.

Foi apresentado um arcabouço para o projeto de operadores de dois níveis para imagens binárias. $\mathrm{O}$ método proposto contribui para o avanço do projeto desses operadores ao automatizar as tarefas de gerar janelas para o projeto dos operadores de primeiro nível e de escolher os operadores utilizados no treinamento do operador de segundo nível. Esse arcabouço usa o método de redução de interações para agrupar pixels das imagens e dividir a área usada pelo operador de dois níveis em subjanelas. Em experimentos, foi comprovado que o método proposto apresenta melhor desempenho que o treinamento de um operador de um único nível. Os resultados obtidos também se aproximam bastante do desempenho de operadores em que a seleção de janelas é feita de maneira manual, ao mesmo tempo evitando o processo custoso de escolher as janelas por tentativa e erro. O método proposto envolve um procedimento de comparação de modelos para determinar a quantidade de operadores de primeiro nível usados no treinamento do operador de segundo nível. Embora o procedimento tenha mostrado a tendência de selecionar modelos mais complexos do que necessário, o operador recomendado pelo procedimento de seleção nunca teve desempenho significativamente diferente do melhor operador encontrado. Esse resultado qualifica o procedimento proposto a determinar o número de operadores em situações em que não é possível validar os resultados em um conjunto independente de testes. Por exemplo, quando o número de amostras é escasso.

O método de seleção de modelos baseia-se numa função que mede a qualidade do modelo, composta por dois termos. Um mede a complexidade do modelo, o outro mede a discrepância (falta de ajuste) entre modelo e conjunto de dados de treinamento. A fórmula dessa função qualidade foi inspirada pelo princípio do comprimento mínimo de descrição (MDL) [Grü07], sem obedecer estritamente 
ao princípio mas conservando a ideia de minimizar medidas de comprimento de descrição. Os termos de complexidade de modelo e de discrepância adotados pelo procedimento proposto são numericamente semelhantes aos adotados no método de Martins e co-autores [MCB06] em problemas de seleção de atributos, porém os pesos relativos atribuídos a cada termo são diferentes. O método apresentado aqui atribui um peso maior ao termo de discrepância. Os três pontos principais do arcabouço proposto (método de seleção de janelas, ordenação dos operadores e escolha do número de operadores) são consistentes entre si, na medida que usam conceitos de teoria da informação para preservar a informação sobre o pixel de saída (rótulo), evitar a redundância entre operadores e controlar a complexidade do modelo. No entanto, ele podem ser recombinados com outras técnicas para atingir o mesmo fim. Por exemplo, o critério CMIM [Fle04] e o procedimento de comparação de modelos podem ser usados junto com outro método de busca no espaço das subjanelas, tal como algoritmos genéticos.

\subsection{Possíveis extensões}

Aqui identificamos algumas possibilidades de extensão deste trabalho. Além dos três métodos citados anteriormente, a técnica de transformação de atributos binários por meio de matrizes de paridade pode dar origem a outros algoritmos, conforme a medida que se pretenda otimizar. Por exemplo, pode-se tentar otimizar a relação entre distâncias inter-classes/intra-classe em problemas de classificação. Em vez de reduzir a magnitude das interações, pode-se tentar reduzir somente a redundância entre atributos (interação negativa), conservando-se as interações positivas.

Uma limitação do método de ICA binária do Capítulo 4 é que o processo de iterar sobre um par de variáveis a cada passo limita as soluções que o algoritmo pode obter, fazendo que ele crie soluções não-ótimas. Uma possibilidade de melhorar o algoritmo é criar outras estratégias de busca sobre o espaço das transformações dos atributos, mas sem aumentar excessivamente a complexidade do algoritmo.

Quanto ao arcabouço de projeto de operadores, os resultados mostram que o procedimento de agregar grupos de pixels para gerar janelas pode ser melhorado se incorporar medidas para incentivar a diversidade espacial das janelas. 


\section{Apêndice A}

\section{Demonstrações}

Este apêndice reúne algumas demonstrações de fórmulas apresentadas no texto principal.

\section{A.1 Fórmulas de Teoria da Informação}

\section{Fórmula da entropia condicional}

$$
\begin{aligned}
H(Y \mid X) & =-\sum_{x \in \mathcal{X}} \sum_{y \in \mathcal{Y}} P(x, y) \log P(y \mid x) \\
& =-\sum_{x \in \mathcal{X}} \sum_{y \in \mathcal{Y}} P(x, y) \log \frac{P(y \mid x) P(x)}{P(x)} \\
& =-\sum_{x \in \mathcal{X}} \sum_{y \in \mathcal{Y}} P(x, y) \log \frac{P(x, y)}{P(x)} \\
& =-\sum_{x \in \mathcal{X}} \sum_{y \in \mathcal{Y}} P(x, y) \log P(x, y)+\sum_{x \in \mathcal{X}} \sum_{y \in \mathcal{Y}} P(x, y) \log P(x) \\
& =-\sum_{x \in \mathcal{X}} \sum_{y \in \mathcal{Y}} P(x, y) \log P(x, y)+\sum_{x \in \mathcal{X}} P(x) \log P(x) \\
& =H(X, Y)-H(X)
\end{aligned}
$$


Equivalência entre $D(P(X, Y) \| P(X) P(Y))$ e $I(X ; Y)$

Partimos da expressão para $D(P(X, Y) \| P(X) P(Y))$ :

$$
D(P(X, Y) \| P(X) P(Y))=\sum_{x \in \mathcal{X}} \sum_{y \in \mathcal{Y}} P(x, y) \log \frac{P(x, y)}{P(x) P(y)} .
$$

Usando a seguinte propriedade:

$$
\log \frac{P(x, y)}{P(x) P(y)}=\log P(x, y)-\log P(x)-\log P(y)
$$

, nós temos que:

$$
\begin{aligned}
D(P(X, Y) \| P(X) P(Y))= & \sum_{x \in \mathcal{X}} \sum_{y \in \mathcal{Y}} P(x, y) \log P(x, y) \\
& -\sum_{x \in \mathcal{X}} \sum_{y \in \mathcal{Y}} P(x, y) \log P(x) \\
& -\sum_{x \in \mathcal{X}} \sum_{y \in \mathcal{Y}} P(x, y) \log P(y)
\end{aligned}
$$

A expressão acima pode ser reescrita como:

$$
\begin{aligned}
D(P(X, Y) \| P(X) P(Y))= & \sum_{x \in \mathcal{X}} \sum_{y \in \mathcal{Y}} P(x, y) \log P(x, y) \\
& -\sum_{x \in \mathcal{X}} \sum_{y \in \mathcal{Y}} P(x) P(y \mid x) \log P(x) \\
& -\sum_{x \in \mathcal{X}} \sum_{y \in \mathcal{Y}} P(y) P(x \mid y) \log P(y) .
\end{aligned}
$$

Reordenando as somatórias, nós temos:

$$
\begin{aligned}
D(P(X, Y) \| P(X) P(Y))= & \sum_{x \in \mathcal{X}} \sum_{y \in \mathcal{Y}} P(x, y) \log P(x, y) \\
& -\sum_{x \in \mathcal{X}} P(x) \log P(x) \sum_{y \in \mathcal{Y}} P(y \mid x) \\
& -\sum_{y \in \mathcal{Y}} P(y) \log P(y) \sum_{x \in \mathcal{X}} P(x \mid y)
\end{aligned}
$$


Lembrando que:

$$
\begin{aligned}
& \sum_{x \in \mathcal{X}} P(x \mid y)=1 \\
& \sum_{y \in \mathcal{Y}} P(y \mid x)=1
\end{aligned}
$$

Nós temos:

$$
\begin{aligned}
D(P(X, Y) \| P(X) P(Y))= & \sum_{x \in \mathcal{X}} \sum_{y \in \mathcal{Y}} P(x, y) \log P(x, y) \\
& -\sum_{x \in \mathcal{X}} P(x) \log P(x) \\
& -\sum_{y \in \mathcal{Y}} P(y) \log P(y)
\end{aligned}
$$

Aplicando a definição de entropia, segue que:

$$
\begin{gathered}
D(P(X, Y) \| P(X) P(Y))=H(X)+H(Y)-H(X, Y) \\
D(P(X, Y) \| P(X) P(Y))=I(X ; Y)
\end{gathered}
$$

\section{Relação entre informação de interação e informação mútua}

$$
\begin{aligned}
I\left(Y ; X_{1} ; X_{2}\right)= & -H(Y)-H\left(X_{1}\right)+H\left(X_{1}, Y\right)-H\left(X_{2}\right)+H\left(X_{2}, Y\right) \\
& +H\left(X_{1}, X_{2}\right)-H\left(X_{1}, X_{2}, Y\right) \\
I\left(Y ; X_{1} ; X_{2}\right)= & -I\left(Y ; X_{1}\right)-H\left(X_{2}\right)+H\left(X_{2}, Y\right) \\
& +H\left(X_{1}, X_{2}\right)-H\left(X_{1}, X_{2}, Y\right) \\
I\left(Y ; X_{1} ; X_{2}\right)= & -I\left(Y ; X_{1}\right)-I\left(Y ; X_{2}\right)+H(Y) \\
& +H\left(X_{1}, X_{2}\right)-H\left(X_{1}, X_{2}, Y\right) \\
I\left(Y ; X_{1} ; X_{2}\right)= & -I\left(Y ; X_{1}\right)-I\left(Y ; X_{2}\right)+ \\
& +I\left(Y ;\left(X_{1}, X_{2}\right)\right)
\end{aligned}
$$




\section{Informação de interação e divergência KL}

A informação de interação corresponde à divergência KL entre $P\left(X_{1}, X_{2}, Y\right)$ e uma aproximação da distribuição conjunta baseada no produto de marginais. Seja $Q\left(X_{1}, X_{2}, Y\right)$ uma função definida por

$$
Q\left(X_{1}, X_{2}, Y\right)=\frac{P\left(X_{1}, X_{2}\right) P\left(X_{1}, Y\right) P\left(X_{2}, Y\right)}{P\left(X_{1}\right) P\left(X_{2}\right) P(Y)} .
$$

A função $Q\left(X_{1}, X_{2}, Y\right)$ não é necessariamente normalizada, ou seja, podemos ter:

$$
\sum_{x_{1} \in \mathcal{X}_{1}} \sum_{x_{2} \in \mathcal{X}_{2}} \sum_{y \in \mathcal{Y}} Q\left(x_{1}, x_{2}, y\right) \neq 1
$$

A divergência KL $D(P \| Q)$ é dada por:

$$
D(P \| Q)=\sum_{\mathcal{X}_{1}, \mathcal{X}_{2}, \mathcal{Y}} P\left(X_{1}, X_{2}, Y\right) \log \frac{P\left(X_{1}\right) P\left(X_{2}\right) P(Y) P\left(X_{1}, X_{2}, Y\right)}{P\left(X_{1}, X_{2}\right) P\left(X_{1}, Y\right) P\left(X_{2}, Y\right)}
$$

Aplicando-se a seguinte propriedade da função logaritmo:

$$
\begin{aligned}
\log \frac{P\left(X_{1}\right) P\left(X_{2}\right) P(Y) P\left(X_{1}, X_{2}, Y\right)}{P\left(X_{1}, X_{2}\right) P\left(X_{1}, Y\right) P\left(X_{2}, Y\right)}= & \log P\left(X_{1}\right)+\log P\left(X_{2}\right)+\log P(Y) \\
& -\log P\left(X_{1}, X_{2}\right)-\log P\left(X_{1}, Y\right) \\
& -\log P\left(X_{2}, Y\right)+\log P\left(X_{1}, X_{2}, Y\right)
\end{aligned}
$$

Pode-se mostrar que:

$$
\begin{aligned}
D(P \| Q)= & -H\left(X_{1}\right)-H\left(X_{2}\right)-H(Y)+H\left(X_{1}, X_{2}\right)+H\left(X_{1}, Y\right) \\
& +H\left(X_{2}, Y\right)-H\left(X_{1}, X_{2}, Y\right) \\
= & I\left(Y ; X_{1} ; X_{2}\right)
\end{aligned}
$$

Como a função $Q\left(X_{1}, X_{2}, Y\right)$ não é necessariamente normalizada a informação de interação pode ser negativa (a divergência KL calculada entre duas distribuições normalizadas é sempre não negativa). 
Quando $I\left(X_{1} ; X_{2} ; Y\right)=0$ temos

$$
P\left(X_{1}, X_{2}, Y\right)=\frac{P\left(X_{1}, X_{2}\right) P\left(X_{1}, Y\right) P\left(X_{2}, Y\right)}{P\left(X_{1}\right) P\left(X_{2}\right) P(Y)}
$$

e

$$
P\left(Y \mid X_{1}, X_{2}\right)=\frac{P\left(X_{1}, Y\right) P\left(X_{2}, Y\right)}{P\left(X_{1}\right) P\left(X_{2}\right) P(Y)}
$$

que pode ser reescrita como:

$$
P\left(Y \mid X_{1}, X_{2}\right)=\frac{P\left(Y \mid X_{1}\right) P\left(Y \mid X_{2}\right)}{P(Y)}
$$




\section{Referências Bibliográficas}

[Ama89] Amari, Shun-ichi: Characteristics of sparsely encoded associative memory. Neural Networks, 2(6):451-457, 1989.

[AS94] Agrawal, Rakesh e Ramakrishnan Srikant: Fast algorithms for mining association rules. In 20th International Conference on Very Large Data Bases, Santiago, Chile, páginas 487-499, setembro 1994.

[BB91] Banon, G. J. F. e J. Barrera: Minimal Representations for Translation-Invariant Set Mappings by Mathematical Morphology. SIAM J. Applied Mathematics, 51(6):1782-1798, dezembro 1991.

[Bel03] Bell, Anthony J: The co-information lattice. In 4th International Symposium on Independent Component Analysis and Blind Signal Separation (ICA2003), Nara, Japan, abril 2003.

[BGDA04] Borgne, Hervé Le, Anne Guérin-Dugué e Anestis Antoniadis: Representation of images for classification with independent features. Pattern recognition letters, 25:141-154, 2004.

[Bis06] Bishop, Christopher M.: Pattern Recognition and Machine Learning. Springer, 2006.

[BJ04] Buntine, Wray e Aleks Jakulin: Applying discrete PCA in data analysis. In Proceedings 20th Conference on Uncetainty in Artificial Intelligence, páginas 59-66, 2004. 
[BMS02] Bartlett, Marian Stewart, Javier Movellan e Terrence Sejnowski: Face recognition by independent component analysis. IEEE Transactions on Neural Networks, 13(6), novembro 2002.

[BMUT97] Brin, Sergey, Rajeev Motwani, Jeffrey D. Ullman e Shalom Tsur: Dynamic itemset counting and implication rules for market basket data. In Proceedings of the 1997 ACM SIGMOD international conference on management of data, páginas 255-264, 1997.

[BR98] Beutelspacher, Albrecht e Ute Rosenbaum: Projective Geometry: From Foundations to Applications. Cambridge University Press, 1998.

[BS95] Bell, Anthony e Terrence J. Sejnowski: An information maximisation approach to blind separation and blind deconvolution. Neural Computation, 7:1129-1159, 1995.

[BS96] Bell, Anthony J. e Terrence J. Sejnowski: Learning the higher-order structure of a natural sound. Network: Computation in Neural Systems, 7, 1996.

[BS97] Bell, Anthony e Terrence J. Sejnowski: The "independent components" of images are edge filters. Vision Research, 37:3327-3338, 1997.

[BTHH00] Barrera, J., R. Terada, R. Hirata Jr e N. S. T. Hirata: Automatic Programming of Morphological Machines by PAC Learning. Fundamenta Informaticae, 41(1-2):229-258, 2000.

[BWHY05] Brown, Gavin, Jeremy Wyatt, Rachel Harris e Xin Yao: Diversity Creation Methods: A Survey and Categorisation. Journal of Information Fusion, 6(1):5-20, 2005.

[CL68] Chow, C. I. e C. N. Liu: Approximating discrete probability distributions with dependence trees. IEEE Transactions on Information Theory, IT-14(3):462-467, maio 1968. 
[Com94] Comon, Pierre: Independent component analysis - a new concept? Signal Processing, 36:287-314, 1994.

[CT91] Cover, Thomas M. e Joy A. Thomas: Elements of Information Theory. Wiley series in telecommunications. John Wiley and Sons, 1991.

[DGL96] Devroye, L., L. Györffi e G. Lugosi: A Probabilistic Theory of Pattern Recognition. Springer-Verlag, 1996.

[Die00] Dietterich, T. G.: Ensemble Methods in Machine Learning. In Kittler, J. e F. Roli (editores): Multiple Classifier Systems: First International Workshop, MCS 2000, volume LNCS 1857 de Lecture Notes in Computer Science, páginas 1-15. Springer-Verlag, 2000.

[Dou92] Dougherty, E. R.: Optimal Mean-Square N-Observation Digital Morphological Filters I. Optimal Binary Filters. CVGIP: Image Understanding, 55(1):36-54, January 1992.

[DP97] Domingos, Pedro e Michael J. Pazzani: On the optimality of the simple bayesian classifier under zero-one loss. Machine Learning, 29(2-3):103-130, 1997. citeseer.ist.psu.edu/article/ domingos97optimality.html.

[DT00] Duin, R.P.W. e D.M.J. Tax: Experiments with classifier combining rules. In J. Kittler, F. Roli (editor): Multiple Classifier Systems (Proc. First International Workshop, MCS 2000), Lecture Notes in Computer Science vol. 1857, páginas 16-29. Springer, 2000. http: //ict.ewi.tudelft.nl/ duin/presentations.html.

[DZC96] Dougherty, E. R., Y. Zhang e Y. Chen: Optimal Iterative Increasing Binary Morphological Filters. Optical Engineering, 35(12):34953507, dezembro 1996.

[FGG97] Friedman, Nir, Dan Geiger e Moises Goldszmidt: Bayesian Network Classifiers. Machine Learning, 1997. 
[Fle04] Fleuret, François: Fast binary feature selection with conditional mutual information. Journal of Machine Learning Research, 5:15311555, 2004.

[Grü05] Grünwald, Peter D.: Advances in Minimum Description Length: Theory and Applications, capítulo A Tutorial Introduction to the Minimum Description Principle. MIT Press, 2005.

[Grü07] Grünwald, Peter D.: The Minimum Description Length Principle. MIT Press, 2007.

[HBD02] Hirata, N. S. T., J. Barrera e E. R. Dougherty: The Incremental Splitting of Intervals Algorithm for the Design of Binary Image Operators. In Talbot, H. e R. Beare (editores): Proceedings of the 6th International Symposium: ISMM 2002, 2002.

[HDB00] Hirata, N. S. T., E. R. Dougherty e J. Barrera: Iterative Design of Morphological Binary Image Operators. Optical Engineering, 39(12):3106-3123, December 2000.

[HH00] Hyvärinen, Aapo e Patrick Hoyer: Emergence of phase and shift invariant features by decomposition of natural images into independent features subspaces. Neural Computation, 12(7):1705-1720, 2000.

[HH01] Himberg, Johan e Aapo Hyvärinen: Independent component analysis for binary data: an experimental study. In Proceedings ICA 2001, páginas 552-556, 2001.

[HH02] Hyvärinen, Aapo e Patrick Hoyer: A multi-layer sparse coding network learns contour coding from natural images. Vision Research, 42(12):1593-1605, julho 2002.

[HHI02] Hyvärinen, Aapo, Patrick Hoyer e Mika Inki: Estimating overcomplete independent component bases for image windows. Journal of Mathematical Imaging and Vision, 17(2), 2002. 
[Hir05] Hirata, N. S. T.: Binary image operator design based on stacked generalization. In Frery, A. C. e M. A. F. Rodrigues (editores): Proceedings of the SIBGRAPI 2005, páginas 63-70, 2005.

[Hir09] Hirata, Nina S. T.: Multi-level training of binary morphological operators. IEEE Transactions on Pattern Analysis and Machine Intelligence, 31(4):707-720, 2009.

[HKO01] Hyvärinen, Aapo, Juha Karhunen e Erkki Oja: Independent Component Analysis. Adaptive and learning systems for signal processing, communications and control. John Wiley and Sons, 2001.

[HO00] Hyvärinen, Aapo e Erkki Oja: Independent component analysis: Algorithms and applications. Neural Networks, 13(4-5):411-430, 2000.

[Hop82] Hopfield, J. J.: Neural networks and physical systems with emergent collective computational properties. Proc. Nat. Acad. Sci., 79:25542558, 1982.

[Hop07] Hopfield, J. J.: Hopfield network. Scholarpedia, 2(5):1977, 2007.

[Hoy04] Hoyer, Patrick: Non-negative matrix factorization with sparseness constraints. Journal of Machine Learning Research, 5:1457 - 1469, 2004.

[HR70] Hellman, Martin E. e Josef Raviv: Probability of error, equivocation, and the Chernoff bound. IEEE Transactions on Information Theory, 16(4):368-372, julho 1970.

[HTF01] Hastie, T., R. Tibshirani e J. Friedman: The Elements of Statistical Learning. Springer-Verlag, 2001.

[Hyv99] Hyvärinen, Aapo: Fast and robust fixed-point algorithms for independent component analysis. IEEE Transactions on Neural Networks, 10(3):626-634, 1999. 
[JB03] Jakulin, Aleks e Ivan Bratko: Analyzing attribute dependencies. In 7th European Conference on Principles and Practice of Knowledge Discovery in Databases (PKDD 2003), volume 2838 de Lecture Notes in Artificial Intelligence, páginas 229-240. Springer, setembro 2003.

[Jor04] Jordan, Michael I.: Graphical models. Statistical Science, 19(1):140-155, 2004.

[JrE03] Jenssen, Robert e Torbjørn Eltoft: ICA filter bank for segmentation of textured images. In 4th International Symposium on Independent Component Analysis and Blind Signal Separation (ICA 2003), Nara, Japan, abril 2003.

[KB06] Kabán, Ata e Ella Bingham: ICA-based binary feature construction. In ICA 2006, 2006.

[KC03] Karvanen, J. e A. Cichocki: Measuring sparseness of noisy signals. In 4th International Symposium on Independent Component Analysis and Blind Signal Separation (ICA 2003), páginas 125-130, Nara, Japan, abril 2003.

[KG05] Krause, Andreas e Carlos Guestrin: Near-optimal value of information in graphical models. In Conference on Uncertainty in Artificial Intelligence (UAI), julho 2005.

[KHDM98] Kittler, J., M. Hatef, R. P.W. Duin e J. Matas: On Combining Classifiers. IEEE Transactions on Pattern Analysis and Machine Intelligence, 20(3):226-239, March 1998.

[KPS ${ }^{+}$08] Krömer, P., J. Platos, V. Snásel, D. Húsek, R. Neruda e A. A. Frolov: Investigating boolean matrix factorization. In S. Z. Chris Ding, Tao Li (editor): KDD 2008 Workshop on Data Mining using Matrices and Tensors, páginas 18-25, 2008. 
[LC03] Liu, Xiuwen e Lei Cheng: Independent spectral representations of images for recognition. Journal of the Optical Society of America A, 20(7), julho 2003.

[Lin91] Lin, J.: Divergence measures based on Shannon entropy. IEEE Transactions on Information Theory, 37(1):145-151, 1991.

[LMI03] Learned-Miller, Erik G. e John W. Fisher III: ICA using spacings estimates of entropy. Journal of Machine Learning Research, 4:12711295, 2003, ISSN 1533-7928.

[Mac03] MacKay, David J. C.: Information Theory, Inference, and Learning Algorithms. Cambridge University Press, 2003. http: //www.cambridge.org/0521642981, Available from http://www.inference.phy.cam.ac.uk/mackay/itila/.

[MCB06] Martins Jr., D. C., R. M. Cesar Jr. e J. Barrera: W-operator window design by minimization of mean conditional entropy. Pattern Anal. Appl., 9(2):139-153, 2006, ISSN 1433-7541.

[McG54] McGill, William J.: Multivariate information transmission. Psychometrika, 19:97-116, 1954.

[Mur01] Murphy, Kevin Patrick: An introduction to graphical models. 2001.

[OF96] Olshausen, Bruno A. e David J. Field: Emergence of simple-cell receptive field properties by learning a sparse code for natural images. Nature, 381:607-609, 1996.

[OF97] Olshausen, Bruno A. e David J. Field: Sparse coding with an overcomplete basis set: a strategy employed by V1? Vision Research, 37(23):3311-3325, 1997.

[OF04] Olshausen, Bruno A e David J Field: Sparse coding of sensory inputs. Current Opinion in Neurobiology, 14(4):481 - 487, 2004, ISSN 0959-4388. http://wWw.sciencedirect. com/science/article/B6VS3-4CX007S-5/2/ 582950d39beefeee4021f6487eca637a. 
[Paj97] Pajunen, P.: Separation of binary sources with less sensors than sources. In Proceedings International Conference on Neural Networks (ICNN-97), páginas 1994-1997, 1997.

[Pec02] Pece, Arthur E. C.: The problem of sparse image coding. Journal of Mathematical Imaging and Vision, 17(2):89-108, 2002.

[SDA98] Sarca, O. V., E. R. Dougherty e J. T. Astola: Secondarily Constrained Boolean Filters. Signal Processing, 71(3):247-263, December 1998.

[SDA99] Sarca, O. V., E.R. Dougherty e J. T. Astola: Two-stage Binary Filters. Electronic Imaging, 8(3):219-232, julho 1999.

[SH06] Singliar, Tomás e Milos Hauskrecht: Noisy-OR component analysis and its application to link analysis. Journal of Machine Learning Research, 7:2189 - 2213, 2006.

[Sha48] Shannon, Claude E.: A mathematical theory of communication. Bell System Technical Journal, 27:379-423 and 623-656, julho 1948.

[SHHJ07] Santos, Carlos Silva, Nina Sumiko Tomita Hirata e Roberto Hirata Junior: Two-stage binary image operator design: an approach based on interaction information. In Falcão, Alexandre Xavier e Hélio Côrtes Vieira Lopes (editores): Proceedings of the Brazilian Symposium on Computer Graphics and Image Processing, 20 (SIBGRAPI). IEEE Computer Society, 2007. http://urlib.net/ sid.inpe.br/sibgrapi@80/2007/09.04.14.31.

[SHJ07] Santos, Carlos Silva e Roberto Hirata Jr.: Exploring window selection strategies for two-level binary image operator design. In Proceedings of the ISMM 2007, volume 2, páginas 41-42. Instituto Nacional de Pesquisas Espaciais (INPE), 2007, ISBN 978-85-17-00035-5. http://urlib.net/dpi .inpe. br/ismme80/2007/07.20.05.17.

[SHJ10] Santos, Carlos Silva, Nina Sumiko Tomita Hirata e Roberto Hirata Junior: An information theory framework for two-stage binary image 
operator design. Pattern Recognition Letters, 31(4):297-306, março 2010.

[SKAG04] Stögbauer, Harald, Alexander Kraskov, Sergey A. Astakhov e Peter Grassberger: Least dependent component analysis based on mutual information. Phys. Rev. E, 70(6), 2004.

[SSZ ${ }^{+}$98] Spellman, Paul T., Gavin Sherlock, Dagger Michael Q. Zhang, Vishwanath R. Iyer, Kirk Anders, Michael B. Eisen, Patrick O. Brown, David Botstein e Bruce Futcher: Comprehensive identification of cell cycle-regulated genes of the yeast saccharomyces cerevisiae by microarray hybridization. Molecular Biology of the Cell, 9(12):32733297, dezembro 1998.

[ST99] Slonim, Noam e Naftali Tishby: Agglomerative information bottleneck. In Advances in Neural Information Processing Systems (NIPS12), 1999.

[SV98] Studeny, M e J Vejnarova: Learning in Graphical Models, capítulo The multiinformation function as a tool for measuring stochastic dependence. Kluwer, 1998.

[Vap98] Vapnik, V. N.: Statistical Learning Theory. John Wiley and Sons, 1998.

[VBH05] Vaquero, D. A., J. Barrera e R. Hirata Jr.: A maximum-likelihood approach for multiresolution $W$-operator design. In Proceedings of the XVIII SIBGRAPI, páginas 71-78, 2005, ISBN 0-7695-2389-7.

[Wo192] Wolpert, D. H.: Stacked generalization. Neural Networks, 5:241259, 1992.

[Yeu02] Yeung, Raymond W: A First Course in Information Theory. Springer Science, 1 a edição, 2002.

[ZL03] Zhang, Harry e Charles X. Ling: A fundamental issue of Naive Bayes. In Xiang, Yang e Brahim Chaib-draa (editores): Canadian Confer- 
ence on AI, volume 2671 de Lecture Notes in Computer Science, páginas 591-595. Springer, 2003, ISBN 3-540-40300-0.

[ZW04] Zhang, Nan e Juyang Weng: Sparse representation from a winnertake-all neural network. In Proc. Int. Joint Conf. on Neural Networks (IJCNN), 2004. 